\title{
Continuidade de atratores para problemas parabólicos semilineares com difusibilidade grande localizada
}

\author{
Karina Schiabel Silva \\ Orientador: Prof. Dr. Alexandre Nolasco de Carvalho \\ Tese apresentada ao Instituto de Ciências Matemáticas e de \\ Computação - ICMC-USP, como parte dos requisitos para \\ obtenção do título de Doutor em Ciências - Matemática.
}

\section{"VERSÃO REVISADA APÓS A DEFESA"}

Data da Defesa:

$30 / 03 / 2006$

Visto do Orientador:

USP - São Carlos

Maio/2006 

Ao meu marido, Ricardo, com amor. 

"Para tudo há um tempo, para cada coisa há um momento debaixo dos céus: tempo para nascer, e tempo para morrer; tempo para plantar, e tempo para arrancar o que foi plantado; tempo para matar, e tempo para sarar; tempo para demolir, e tempo para construir; tempo para chorar, e tempo para rir; tempo para gemer, e tempo para dançar; tempo para atirar pedras, e tempo para ajuntá-las; tempo para dar abraços, e tempo para apartar-se. Tempo para procurar, e tempo para perder; tempo para guardar, e tempo para jogar fora; tempo para rasgar, e tempo para costurar; tempo para calar, e tempo para falar; tempo para amar, e tempo para odiar; tempo para a guerra, e tempo para a paz”.

(Ecle 3, 1-8) 



\section{Agradecimentos*}

Não obstante sejam muitas as pessoas a quem devo agradecer, não posso deixar de atribuir especial merecimento a algumas delas.

Ao meu marido, Ricardo Parreira da Silva, não somente pela valiosa colaboração neste trabalho, mas principalmente pelo amor, carinho, atenção e compreensão diante de meus desacertos.

À minha família, em especial aos meus pais, José Maria e Ana Maria Schiabel, e à minha irmã, Daniela Schiabel, pelo carinho e torcida constantes.

A Ariane Di Tullio, Daniel Mancini, Érica Filletti, Erika Cappellini, Esdras Costa, Fábio Nascimento, Gizele Martins, Marcelo Nascimento e Paula Di Tullio, pela amizade preciosa e fiel de tantos anos.

Aos colegas de curso, funcionários e docentes do ICMC-USP.

Ao Prof. José Gaspar Ruas Filho, pelo primeiro incentivo e pelos conselhos edificantes, e à Prof $^{\mathrm{a}}$. Vera Lúcia Carbone, pela amizade e também pela importante contribuição na realização deste trabalho.

Particularmente, ao Prof. Alexandre Nolasco de Carvalho, quero não apenas agradecer pela prontidão, incentivo e paciência sempre demonstrados em sua orientação, mas também registrar minha admiração por sua inteligência, disciplina e seriedade profissional.

\footnotetext{
* Este trabalho foi parcialmente financiado pela CAPES e pelo CNPq.
} 



\section{Resumo}

Neste trabalho estudamos o comportamento assintótico de problemas parabólicos semilineares do tipo

$$
u_{t}-\operatorname{div}(p(x) \nabla u)+\lambda u=h(u)
$$

em um domínio limitado e suave $\Omega \subset \mathbb{R}^{n}$, com condições de Neumann na fronteira, quando o coeficiente de difusão $p$ torna-se grande em uma sub-região $\Omega_{0}$ que é interior ao domínio físico $\Omega$. Provamos que, sob determinadas hipóteses, a família de atratores se comporta semicontinuamente inferior e superiormente quando a difusão explode em $\Omega_{0}$. 



\section{Abstract}

In this work we study the asymptotic behavior of semilinear parabolic problems of the form

$$
u_{t}-\operatorname{div}(p(x) \nabla u)+\lambda u=h(u)
$$

in a bounded smooth domain $\Omega \subset \mathbb{R}^{n}$ and Neumann boundary conditions when the diffusion coefficient $p$ becomes large in a subregion $\Omega_{0}$ which is interior to the physical domain $\Omega$. We prove, under suitable assumptions, that the family of attractors behave upper and lowersemicontinuously as the diffusion blows up in $\Omega_{0}$. 



\section{Sumário}

Agradecimentos $\quad$ i

Resumo iii

$\begin{array}{lll}\text { Abstract } & \text { v }\end{array}$

$\begin{array}{ll}\text { Introdução } & 1\end{array}$

\begin{tabular}{lll}
\hline 1 & Resultados preliminares & 7
\end{tabular}

1.1 Semigrupos e seus geradores $\ldots \ldots \ldots \ldots \ldots$. . . . . . . . . . .

$1.1 .1 \quad$ Operadores setoriais $\ldots \ldots \ldots \ldots 11$

1.1.2 Potências fracionárias de operadores setoriais . . . . . . . . . . . . . 11

1.1.3 Problema de Cauchy abstrato . . . . . . . . . . . . . . . . . 12

1.2 Teoria geral de atratores $\ldots \ldots \ldots \ldots \ldots$

1.2.1 Existência e limitação . . . . . . . . . . . . . . . . . . . . . . . 14

1.2.2 Caracterização de atratores para sistemas gradientes $\ldots . . \ldots .18$

1.2.3 Continuidade de atratores . . . . . . . . . . . . . . . . 19

1.3 Teoremas de imersões de Sobolev . . . . . . . . . . . . . . . . . . . 21

1.3.1 Teorema do Traço . . . . . . . . . . . . . . . . 22

2 Equações de reação e difusão com condições de fronteira não-lineares 25

2.1 Problemas parabólicos com condições de fronteira não-lineares . . . . . . . . . 25

2.1 .1 Existências local e global de solução . . . . . . . . . . . . . . . . . 28

2.1.2 Existência de atratores e estimativas uniformes . . . . . . . . . . . 31

2.2 Problema perturbado . . . . . . . . . . . . . . 32

2.3 Problema limite . . . . . . . . . . . . . . . . . . 36 
3 Teoria linear $\quad 41$

3.1 Convergência compacta do resolvente . . . . . . . . . . . . . . . 43

3.2 Continuidade do espectro . . . . . . . . . . . . . . . . . 47

3.3 Convergência dos semigrupos lineares . . . . . . . . . . . . . . 53

4 Continuidade dos atratores $\quad 57$

4.1 Continuidade dos semigrupos não-lineares . . . . . . . . . . . . . . 58

4.2 Semicontinuidade superior dos atratores . . . . . . . . . . . . 63

4.3 Continuidade dos conjuntos de equilíbrio . . . . . . . . . . . . 64

4.3 .1 Linearização em torno de um ponto de equilíbrio . . . . . . . . . . . 65

4.4 Continuidade das variedades instáveis $\ldots \ldots$. . . . . . . . . . . . . . 77

4.5 Semicontinuidade inferior dos atratores . . . . . . . . . . . . . . . 89

\begin{tabular}{ll}
\hline Referências bibliográficas & 91
\end{tabular}

$\begin{array}{ll}\text { Índice remissivo } & 93\end{array}$ 


\section{Introdução}

Sejam $\Omega \subset \mathbb{R}^{n}$ um domínio limitado e suave, $\Gamma=\partial \Omega$, e $\varepsilon \in\left(0, \varepsilon_{0}\right]$ um parâmetro. Neste trabalho estudamos o comportamento das soluções da família de equações parabólicas

$$
\begin{cases}u_{t}^{\varepsilon}-\operatorname{div}\left(p_{\varepsilon}(x) \nabla u^{\varepsilon}\right)+\lambda u^{\varepsilon}=f\left(u^{\varepsilon}\right) & \operatorname{em} \Omega \\ p_{\varepsilon}(x) \frac{\partial u^{\varepsilon}}{\partial \vec{n}}=g\left(u^{\varepsilon}\right) & \operatorname{em~} \Gamma \\ u^{\varepsilon}(0)=u_{0}^{\varepsilon}, & \end{cases}
$$

quando $\varepsilon \rightarrow 0, \lambda>0, f, g \in \mathscr{C}^{2}(\mathbb{R})$.

O parâmetro $\varepsilon$ representa o fato que, quando $\varepsilon \rightarrow 0$, a difusibilidade $p_{\varepsilon}$ se torna muito grande em uma região $\Omega_{0}$, que é interior ao domínio $\Omega$. Esta situação pode ser encontrada, por exemplo, em modelos de condução de calor em materiais compostos, onde o calor pode se difundir muito mais rapidamente em uma parte da região do que em outras.

Sejam $\Omega_{0}$ um sub-domínio suave de $\Omega$, com $\bar{\Omega}_{0} \subset \Omega$ e $m$ um inteiro positivo tal que $\Omega_{0}=\bigcup_{i=1}^{m} \Omega_{0, i}$, onde $\Omega_{0, i}$ são sub-domínios suaves e conexos de $\Omega$ com $\bar{\Omega}_{0, i} \cap \bar{\Omega}_{0, j}=\varnothing$, para $i \neq j$. Denotemos por $\Omega_{1}=\Omega \backslash \bar{\Omega}_{0}$, e $\Gamma_{0, i}=\partial \Omega_{0, i}, \Gamma_{0}=\bigcup_{i=1}^{m} \Gamma_{0, i}$ as fronteiras de $\Omega_{0, i}$ e $\Omega_{0}$, respectivamente. Notemos que $\partial \Omega_{1}=\Gamma \cup \Gamma_{0}$.

Os coeficientes de difusão $p_{\varepsilon}: \Omega \subset \mathbb{R}^{n} \rightarrow \mathbb{R}$ são funções regulares e limitadas em $\Omega$, satisfazendo

$$
0<m_{0} \leqslant p_{\varepsilon}(x) \leqslant M_{\varepsilon}
$$

para todo $x \in \Omega$ e $0<\varepsilon \leqslant \varepsilon_{0}$. Também assumimos que a difusão é grande em $\Omega_{0}$ quando $\varepsilon \rightarrow 0$, mais precisamente,

$$
p_{\varepsilon}(x) \rightarrow\left\{\begin{array}{cl}
p_{0}(x), & \text { uniformemente sobre } \Omega_{1},\left(p_{0} \in \mathscr{C}^{1}\left(\bar{\Omega}_{1},(0, \infty)\right)\right) \\
\infty, & \text { uniformemente sobre subconjuntos compactos de } \Omega_{0} .
\end{array}\right.
$$

Uma vez que difusibilidade grande implica uma rápida redistribuição das não-homogeneidades espaciais, para valores pequenos de $\varepsilon$, a solução do problema (1) deverá ser aproximadamente espacialmente constante em $\Omega_{0}$. Por esta razão, suponhamos que $u^{\varepsilon}$ convirja para 
alguma função $u$, em algum sentido, e que assuma um valor espacialmente constante sobre $\Omega_{0}$, que denotaremos por $u_{\Omega_{0}}(t)$.

Notemos agora que se a função limite $u$ está em $H^{1}(\Omega)$, seu valor constante sobre $\Omega_{0}, u_{\Omega_{0}}(t)$, pode não ser arbitrário. Também na fronteira $\Gamma_{0}=\partial \Omega_{0}$, devemos ter $u_{\Gamma_{0}}=u_{\Omega_{0}}(t)$.

Sobre $\Omega_{1}$, temos

$$
u_{t}^{\varepsilon}-\operatorname{div}\left(p_{\varepsilon}(x) \nabla u^{\varepsilon}\right)+\lambda u^{\varepsilon}=f\left(u^{\varepsilon}\right) .
$$

Das propriedades de convergência da função $p_{\varepsilon}(x)$ é razoável termos no limite

$$
u_{t}-\operatorname{div}\left(p_{0}(x) \nabla u\right)+\lambda u=f(u), \text { para } u \in H^{1}(\Omega) .
$$

Sobre $\Omega_{0}$, temos

$$
\int_{\Omega_{0}} u_{t}^{\varepsilon}(x, t) d x-\int_{\Omega_{0}} \operatorname{div}\left(p_{\varepsilon}(x) \nabla u^{\varepsilon}\right) d x+\int_{\Omega_{0}} \lambda u^{\varepsilon}(x, t) d x=\int_{\Omega_{0}} f\left(u^{\varepsilon}(x, t)\right) d x .
$$

Do Teorema da Divergência segue que

$$
\int_{\Omega_{0}} \operatorname{div}\left(p_{\varepsilon}(x) \nabla u^{\varepsilon}\right) d x=-\int_{\Gamma_{0}} p_{\varepsilon}(x)\left\langle\nabla u^{\varepsilon}, \vec{n}\right\rangle d x,
$$

onde $\vec{n}$ é o vetor normal unitário interior em $\Omega_{0}$ na fronteira $\Gamma_{0}$ de $\Omega_{0}$, e assim

$$
\int_{\Omega_{0}} u_{t}^{\varepsilon}(x, t) d x+\int_{\Gamma_{0}} p_{\varepsilon}(x) \frac{\partial u^{\varepsilon}}{\partial \vec{n}} d x+\int_{\Omega_{0}} \lambda u^{\varepsilon}(x, t) d x=\int_{\Omega_{0}} f\left(u^{\varepsilon}(x, t)\right) d x .
$$

Passando ao limite quando $\varepsilon \rightarrow 0$, obtemos

$$
\dot{u}_{\Omega_{0}}(t)\left|\Omega_{0}\right|+\int_{\Gamma_{0}} p_{0}(x) \frac{\partial u}{\partial \vec{n}} d x+\int_{\Omega_{0}} \lambda d x u_{\Omega_{0}}(t)=\left|\Omega_{0}\right| f\left(u_{\Omega_{0}}(t)\right) .
$$

Dividindo ambos os lados por $\left|\Omega_{0}\right|$, temos a seguinte equação diferencial ordinária:

$$
\dot{u}_{\Omega_{0}}(t)+\frac{1}{\left|\Omega_{0}\right|}\left(\int_{\Gamma_{0}} p_{0}(x) \frac{\partial u}{\partial \vec{n}} d x+\int_{\Omega_{0}} \lambda d x u_{\Omega_{0}}(t)\right)=f\left(u_{\Omega_{0}}(t)\right),
$$

que é a equação relacionando o fluxo de calor de $\Omega_{1}$ para $\Omega_{0}$ através de $\Gamma_{0}$ com o calor total absorvido em $\Omega_{0}$ e o calor colocado em $\Omega_{0}$. Também relaciona o valor de $u_{\Omega_{0}}$ com os valores de $u$ em $\Omega_{1}$, através do termo integral ao longo de $\Gamma_{0}$.

Com estas considerações heurísticas, e considerando que no limite trabalhamos com o espaço das funções constantes sobre $\Omega_{0}$, escrevemos o problema limite da seguinte forma 


$$
\begin{cases}u_{t}-\operatorname{div}\left(p_{0}(x) \nabla u\right)+\lambda u=f(u) & \text { em } \Omega_{1} \\ u_{\mid \Omega_{0, i}}:=u_{\Omega_{0, i}} & \text { em } \Omega_{0, i}, i=1, \ldots, m \\ \dot{u}_{\Omega_{0, i}}+\frac{1}{\left|\Omega_{0, i}\right|}\left[\int_{\Gamma_{0, i}} p_{0}(x) \frac{\partial u}{\partial \vec{n}} d x+\int_{\Omega_{0, i}} \lambda d x u_{\Omega_{0, i}}\right]=f\left(u_{\Omega_{0, i}}\right) & i=1, \ldots, m \\ p_{0}(x) \frac{\partial u}{\partial \vec{n}}=g(u) & \text { em } \Gamma \\ u(0)=u_{0} . & \end{cases}
$$

É importante observarmos que o problema (1) é uma perturbação singular do problema (3) onde fazemos o coeficiente de difusão grande em sub-regiões do domínio.

Consideramos

$$
\mathscr{D}\left(A_{\varepsilon}\right)=\left\{u \in \mathrm{H}^{1}(\Omega):-\operatorname{div}\left(p_{\varepsilon}(x) \nabla u\right) \in \mathrm{L}^{2}(\Omega), \frac{\partial u}{\partial \vec{n}}=0 \mathrm{em} \Gamma\right\},
$$

e, para $u \in \mathscr{D}\left(A_{\varepsilon}\right)$

$$
A_{\varepsilon} u=-\operatorname{div}\left(p_{\varepsilon}(x) \nabla u\right)+\lambda u
$$

Seja ainda

$$
\mathscr{D}\left(A_{0}\right)=\left\{u \in \mathrm{H}_{\Omega_{0}}^{1}(\Omega):-\operatorname{div}\left(p_{0}(x) \nabla u\right) \in \mathrm{L}^{2}\left(\Omega_{1}\right), \frac{\partial u}{\partial \vec{n}}=0 \mathrm{em} \Gamma\right\},
$$

e, para $u \in \mathscr{D}\left(A_{0}\right)$,

$$
\begin{aligned}
A_{0} u & =\left(-\operatorname{div}\left(p_{0}(x) \nabla u\right)+\lambda u\right) \chi_{\Omega_{1}} \\
& +\sum_{i=1}^{m} \frac{1}{\left|\Omega_{0, i}\right|}\left(\int_{\Gamma_{0, i}} p_{0}(x) \frac{\partial u}{\partial \vec{n}} d x+\int_{\Omega_{0, i}} \lambda u_{\Omega_{0, i}} d x\right) \chi_{\Omega_{0, i}},
\end{aligned}
$$

sendo $\chi_{B}$ a função característica do conjunto $B$ e, em geral, $Y_{\Omega_{0}}=\left\{u \in Y: u\right.$ é constante em $\left.\Omega_{0}\right\}$.

Mostramos que os problemas (1) e (3) possuem soluções globalmente definidas em $H^{1}(\Omega)$ e $H_{\Omega_{0}}^{1}(\Omega)$, respectivamente. Além disso, lançando mão de algumas hipóteses de crescimento e sinal sobre as não-linearidades $f$ e $g$ e ainda uma condição de dissipatividade, provamos que estes problemas possuem atratores globais $\mathscr{A}_{\varepsilon}$ e $\mathscr{A}_{0}$, que são uniformemente limitados em $H^{1}(\Omega)$ e $H_{\Omega_{0}}^{1}(\Omega)$, respectivamente, e também em $L^{\infty}(\Omega)$. Este fato nos permite cortar as nãolinearidades $f$ e $g$ de tal forma estas tornem-se limitadas e suas derivadas até segunda ordem 
também sejam limitadas. Sendo assim, assumimos, sem perda de generalidade, que existem constantes $C_{f}>0$ e $C_{g}>0$ satisfazendo

$$
\left|f^{(j)}(s)\right| \leqslant C_{f},\left|g^{(j)}(s)\right| \leqslant C_{g}, \text { para todo } s \in \mathbb{R}, j=0,1,2 \text {. }
$$

Em [8], Carbone e Ruas-Filho mostrararam a continuidade dos atratores para $n=1$. No caso do problema unidimensional, é possível mostrar que autovalores consecutivos $\lambda_{n}^{0}$ e $\lambda_{n+1}^{0}$ do problema linear de (3) estão separados por intervalos de comprimento $\lambda_{n+1}^{0}-\lambda_{n}^{0}$, que divergem para $+\infty$ quando $n \rightarrow \infty$ (propriedade chamada de gap) e que a convergência dos autovalores e autovetores do problema linear de (1) para os autovalores e autovetores do problema (3) garantem a existência de variedades invariantes (de mesma dimensão) para estes problemas e, por fim, a convergência $C^{1}$ dessas variedades permitem concluir a continuidade dos atratores para estes problemas. Entretanto, a técnica utilizada em [8] não pode ser aplicada para $n>1$, uma vez que não podemos garantir a existência do gap.

Para $n \geqslant 1$, a semicontinuidade superior dos atratores foi provada em [5]. Arrieta, Carvalho e Rodríguez-Bernal estudaram a caracterização dos problemas de autovalores associados a (1) e (3).

Com o objetivo de provar a continuidade dos atratores, organizamos este trabalho da seguinte forma: o Capítulo 1 contém definições, notações e resultados preliminares que são utilizados no decorrer dos demais capítulos. Na Seção 1.2, em particular, versamos sobre a teoria geral de atratores. Provamos que o fato da não-linearidade ser globalmente limitada nos permite concluir a existência de atratores globais e sua limitação uniforme nos espaços de potências fracionárias.

Estudamos, no Capítulo 2, as equações de reação-difusão com condições de fronteira nãolineares. O capítulo é constituído de alguns resultados abstratos, baseados principalmente em [5] e [10], das formulações das equações abstratas associadas aos problemas (1) e (3) e do estudo das propriedades dos operadores.

Apresentamos no Capítulo 3 definições e resultados úteis na demonstração da convergência compacta dos operadores resolventes. Estudamos em seguida o comportamento espectral dos operadores lineares e, finalmente, mostramos um teorema de aproximação do tipo Trotter-Kato para os semigrupos lineares.

Reservamos o Capítulo 4 para tratarmos da continuidade dos atratores. Utilizando os resultados obtidos no capítulo anterior e a Fórmula da Variação das Constantes, provamos que a família de semigrupos não-lineares $\left\{T_{\varepsilon}(t): t \geqslant 0\right\}$ associada a (3) é contínua em $\varepsilon=0$ em inter- 
valos compactos de $(0, \infty)$. Deste fato segue facilmente a semicontinuidade superior da família de atratores. Em seguida reunimos os primeiros resultados imprescindíveis à demonstração da semicontinuidade inferior dos atratores, a saber, a continuidade dos conjuntos de equilíbrio e das variedades instáveis. Assumimos que todos os pontos de equilíbrio do problema (3) são hiperbólicos. Mostramos então que o conjunto das soluções de equilíbrio de (3), que denotamos $\mathscr{E}_{0}$, tem cardinalidade finita $n_{0}$, com elementos distintos $u_{1}, \ldots, u_{n_{0}}$. Em seguida, mostramos que existe $\bar{\varepsilon} \in\left(0, \varepsilon_{0}\right]$ tal que o problema (1) tem exatamente $n_{0}$ soluções de equilíbrio, denotadas por $u_{1}^{\varepsilon}, \ldots, u_{n_{0}}^{\varepsilon}$, para $\varepsilon \in(0, \bar{\varepsilon}]$. Para estas soluções, obtemos a convergência $u_{j}^{\varepsilon} \rightarrow u_{j}$ em $H^{1}(\Omega)$ quando $\varepsilon \rightarrow 0$, para $j=1, \ldots, n_{0}$. Finalizando o capítulo, lançamos mão da continuidade dos semigrupos lineares para mostrarmos que as variedades instáveis locais $\mathrm{W}_{\varepsilon, \text { loc }}^{u}\left(u_{j}^{\varepsilon}\right)$ dos pontos de equilíbrio $u_{j}^{\varepsilon}$ são contínuas em $\varepsilon=0$. Em seguida mostramos que (1) e (3) têm estrutura gradiente para, finalmente, demonstrarmos a semicontinuidade inferior da família de atratores. 


\section{Capítulo}

\section{Resultados preliminares}

O objetivo deste capítulo é reunir alguns dos resultados que serão utilizados nos capítulos subseqüentes. Reservamos as duas primeiras seções deste capítulo para introduzir definições e combinar resultados clássicos da teoria de semigrupos e de atratores. Tais resultados podem ser encontrados principalmente em Carvalho [12], Hale [14] e Pazy [17]. Na Seção 1.3, estudamos os Teoremas de Imersão de Sobolev e o Teorema do Traço, que serão muito úteis no estudo de problemas com condições de fronteira não-lineares.

\subsection{Semigrupos e seus geradores}

Em toda a seção, $X$ denotará um espaço de Banach sobre um corpo $\mathbb{K}$, e $\mathscr{L}(X)$ o espaço dos operadores lineares e contínuos em $X$, munido da norma

$$
\|A\|_{\mathscr{L}(X)}=\sup _{\substack{x \in X \\\|x\|_{X}=1}}\|A x\|_{X} .
$$

Denotaremos ainda por $X^{*}:=\mathscr{L}(X, \mathbb{K})$ o dual topológico de $X$.

Um semigrupo fortemente contínuo (ou um $C_{0}$-semigrupo) de operadores lineares em $X$ é uma família $\{T(t): t \geqslant 0\} \subset \mathscr{L}(X)$ tal que

(i) $T(0)=I_{X}$.

(ii) $T(t+s)=T(t) T(s)$, para quaisquer $t, s \geqslant 0$.

(iii) $\|T(t) x-x\|_{X} \rightarrow 0$ quando $t \rightarrow 0^{+}$, para todo $x \in X$.

Todo semigrupo fortemente contínuo possui uma limitação exponencial que é dada no teorema a seguir. 
Teorema 1.1. Suponhamos que $\{T(t): t \geqslant 0\} \subset \mathscr{L}(X)$ seja um semigrupo fortemente contínuo. Então existem $M \geqslant 1$ e $\omega \in \mathbb{R}$ tais que

$$
\|T(t)\|_{\mathscr{L}(X)} \leqslant M e^{\omega t}, \quad \text { para todo } t \geqslant 0 .
$$

Se $\{T(t): t \geqslant 0\} \subset \mathscr{L}(X)$ é um semigrupo fortemente contínuo de operadores lineares, seu gerador infinitesimal é o operador definido por $A: \mathscr{D}(A) \subset X \rightarrow X$, onde

$$
\mathscr{D}(A)=\left\{x \in X: \lim _{t \rightarrow 0^{+}} \frac{T(t) x-x}{t} \text { existe }\right\}, \quad A x=\lim _{t \rightarrow 0^{+}} \frac{T(t) x-x}{t} .
$$

Notemos que o operador $A$ é fechado e densamente definido. Definimos o conjunto resolvente $\rho(A)$ de um operador $A$ como sendo

$$
\rho(A)=\{\lambda \in \mathbb{C}:(\lambda-A) \text { é contínuo, injetivo e sobrejetivo }\} .
$$

O conjunto complementar de $\rho(A)$ é chamado espectro de $A$ e é denotado por $\sigma(A)$.

O resultado a seguir reúne alguns fatos importantes sobre semigrupos fortemente contínuos.

Teorema 1.2. Suponhamos que $\{T(t): t \geqslant 0\} \subset \mathscr{L}(X)$ seja um semigrupo fortemente contínuo.

(i) Para qualquer $x \in X, t \rightarrow T(t) x$ é contínuo para $t \geqslant 0$.

(ii) $t \rightarrow\|T(t)\|_{\mathscr{L}(X)}$ é semicontínua inferiormente e, portanto, mensurável.

(iii) Se A é o gerador infinitesimal de T(t), então A é densamente definido e fechado. Para $x \in \mathscr{D}(A), t \mapsto T(t) x$ é continuamente diferenciável $e$

$$
\frac{d}{d t} T(t) x=A T(t) x=T(t) A x, \quad t>0
$$

(iv) $\bigcap_{m \geqslant 1} \mathscr{D}\left(A^{m}\right)$ é denso em $X$.

(v) Para $\operatorname{Re} \lambda>\beta$ e $\beta$ dado no Teorema 1.1 $\lambda$ está no resolvente $\rho(A)$ de A e

$$
(\lambda-A)^{-1} x=\int_{0}^{\infty} e^{-\lambda t} T(t) x d t, \quad \text { para todo } x \in X
$$


Teorema 1.3 (Hille-Yosida). Suponhamos que $A: \mathscr{D}(A) \subset X \rightarrow X$ seja um operador linear. Então as seguintes afirmações são equivalentes:

(i) A é o gerador infinitesimal de um semigrupo fortemente contínuo $\{T(t): t \geqslant 0\} \subset \mathscr{L}(X)$ tal que

$$
\|T(t)\|_{\mathscr{L}(X)} \leqslant e^{\omega t}, \quad \text { para todo } t \geqslant 0
$$

(ii) A é um operador linear fechado, densamente definido, cujo conjunto resolvente contém $(\omega, \infty) e$

$$
\left\|(\lambda-A)^{-1}\right\|_{\mathscr{L}(X)} \leqslant \frac{1}{|\lambda-\omega|}, \quad \text { para todo } \lambda>\omega .
$$

Sejam $X$ um espaço de Banach sobre $\mathbb{K}$ com norma $\|\cdot\|_{X}$ e $X^{*}=\mathscr{L}(X, \mathbb{K})$ o seu dual topológico, munido da norma usual $\|\cdot\|_{X^{*}}\left(\left\|x^{*}\right\|_{X^{*}}=\sup \left\{\operatorname{Re}\left\langle x^{*}, x\right\rangle:\|x\|_{X}=1\right\}\right)$. A aplicação dualidade $J: X \rightarrow 2^{X^{*}}$ é uma função multívoca definida por

$$
J(x)=\left\{x^{*} \in X^{*}: \operatorname{Re}\left\langle x^{*}, x\right\rangle=\|x\|_{X}^{2},\left\|x^{*}\right\|_{X^{*}}=\|x\|_{X}\right\} .
$$

Temos ainda que $J(x) \neq \varnothing$, pelo Teorema de Hahn-Banach.

Um operador linear $A: \mathscr{D}(A) \subset X \rightarrow X$ é dissipativo se para cada $x \in \mathscr{D}(A)$ existe $x^{*} \in J(x)$ tal que $\operatorname{Re}\left\langle x^{*}, A x\right\rangle \leqslant 0$.

Lema 1.4. O operador linear A é dissipativo se, e somente se,

$$
\|(\lambda-A) x\|_{X} \geqslant \lambda\|x\|_{X}
$$

para quaisquer $x \in \mathscr{D}(A)$ e $\lambda>0$.

Teorema 1.5 (Lumer-Phillips). Suponhamos que A seja um operador linear densamente definido em um espaço de Banach X.

(i) Se Aé o gerador infinitesimal de um semigrupo fortemente contínuo de contrações, então $A$ é dissipativo (de fato, $\operatorname{Re}\left\langle x^{*}, A x\right\rangle \leqslant 0$ para todo $x^{*} \in J(x)$ e para todo $\left.x \in \mathscr{D}(A)\right) e$ $R(\lambda-A)=X$ para algum $\lambda>0$.

(ii) Se A é dissipativo e $R\left(\lambda_{0}-A\right)=X$ para algum $\lambda_{0}>0$, então A é o gerador de um semigrupo fortemente contínuo de contrações. 
A seguir recordamos a definição de operadores adjuntos. Seja $X$ um espaço de Banach com dual $X^{*}$. Seja $S: \mathscr{D}(S) \subset X \rightarrow X$ um operador linear com domínio denso. O adjunto $S^{*}: \mathscr{D}\left(S^{*}\right) \subset X^{*} \rightarrow X^{*}$ de $S$ é o operador linear definido por: $\mathscr{D}\left(S^{*}\right)$ é o conjunto dos $x^{*} \in X^{*}$ para os quais existe $y^{*} \in X^{*}$ com

$$
\left\langle x^{*}, S x\right\rangle=\left\langle y^{*}, x\right\rangle \quad \text { para todo } x \in \mathscr{D}(S) .
$$

Se $x^{*} \in \mathscr{D}\left(S^{*}\right)$, então $y^{*}=S^{*} x^{*}$, onde $y^{*}$ é o elemento de $X^{*}$ satisfazendo (1.2). Notemos que, sendo $\mathscr{D}(S)$ denso em $X$, existe no máximo um $y^{*} \in X^{*}$ para o qual (1.2) vale.

Seja $H$ um espaço de Hilbert com produto interno $\langle\cdot, \cdot\rangle$. Identificamos $H$ e $H^{*}$ e denotamos ambos por $H$.

Seja $H$ um espaço de Hilbert com produto interno $\langle\cdot, \cdot\rangle$. Um operador $A: \mathscr{D}(A) \subset H \rightarrow H$ é simétrico se $\overline{\mathscr{D}(A)}=H$ e $A \subset A^{*}$, ou seja, $\langle A x, y\rangle=\langle x, A y\rangle$ para quaisquer $x, y \in \mathscr{D}(A)$. $A$ é auto-adjunto se $A=A^{*}$.

Corolário 1.6. Seja A um operador linear fechado e densamente definido. Se A e A* são dissipativos, então A é o gerador infinitesimal de um semigrupo fortemente contínuo de contrações sobre $X$.

Em muitos exemplos, a técnica utilizada para verificar as estimativas espectrais necessárias para se garantir que um operador $A$ é o gerador de um semigrupo fortemente contínuo de operadores é a determinação da chamada imagem numérica, que definimos a seguir.

Se $A$ é um operador linear em um espaço de Banach complexo $X$, a sua imagem numérica $W(A)$ é o conjunto

$$
W(A):=\left\{\left\langle x^{*}, A x\right\rangle: x \in \mathscr{D}(A),\|x\|_{X}=1, x^{*} \in X^{*},\left\|x^{*}\right\|_{X^{*}}=1,\left\langle x^{*}, x\right\rangle=1\right\} .
$$

No caso em que $X$ é um espaço de Hilbert, $W(A)=\left\{\langle A x, x\rangle: x \in \mathscr{D}(A),\|x\|_{X}=1\right\}$.

Teorema 1.7. Sejam $A: \mathscr{D}(A) \subset X \rightarrow X$ um operador fechado densamente definido, $W(A)$ sua imagem numérica, $\Sigma$ um subconjunto aberto e conexo em $\mathbb{C} \backslash W(A)$. Se $\lambda \notin \overline{W(A)}$, então $\lambda-A$ é injetivo, tem imagem fechada e satisfaz.

$$
\|(\lambda-A) x\|_{X} \geqslant \operatorname{dist}(\lambda, W(A))\|x\|_{X}
$$

Além disso, se $\rho(A) \cap \Sigma \neq \varnothing$, então $\rho(A) \supset \Sigma e$

$$
\left\|(\lambda-A)^{-1}\right\|_{\mathscr{L}(X)} \leqslant \frac{1}{\operatorname{dist}(\lambda, W(A))}, \quad \text { para todo } \lambda \in \Sigma \text {. }
$$




\subsubsection{Operadores setoriais}

Dizemos que um operador linear $A$ em um espaço de Banach $X$ é um operador setorial se ele é fechado, densamente definido e existem $0<\phi<\frac{\pi}{2}, M \geqslant 1$ e $a \in \mathbb{R}$ tais que

$$
\Sigma_{a, \phi}=\{\lambda \in \mathbb{C}: \phi \leqslant|\arg (\lambda-a)| \leqslant \pi, \lambda \neq a\}
$$

está no conjunto resolvente de $A$ e

$$
\left\|(\lambda-A)^{-1}\right\|_{\mathscr{L}(X)} \leqslant \frac{M}{|\lambda-a|}, \quad \text { para todo } \lambda \in \Sigma_{a, \phi} .
$$

Seja $\Delta=\left\{z: \phi_{1}<\arg z<\phi_{2}, \phi_{1}<0<\phi_{2}\right\}$ e, para $z \in \Delta$, seja $T(z)$ um operador linear limitado. A família $\{T(z): z \in \Delta\}$ é um semigrupo analítico sobre $\Delta$ se

(i) $z \mapsto T(z)$ é analítica em $\Delta$.

(ii) $T(0)=I$ e $\lim _{z \rightarrow 0} T(z) x=x$, para cada $x \in X$.

(iii) $T\left(z_{1}+z_{2}\right)=T\left(z_{1}\right) T\left(z_{2}\right)$, quaisquer que sejam $z_{1}, z_{2} \in \Delta$.

Teorema 1.8. Se A é um operador setorial, então - A é o gerador infinitesimal de um semigrupo analítico $\left\{e^{-A t}: t \geqslant 0\right\}$,

$$
e^{-A t}=\frac{1}{2 \pi i} \int_{\Gamma} e^{\lambda t}(\lambda+A)^{-1} d \lambda
$$

sendo $\Gamma$ contorno em $\rho(-A)$, com arg $\lambda \rightarrow \pm \theta$ quando $|\lambda| \rightarrow \infty$, para algum $\frac{\pi}{2}<\theta<\pi$.

\subsubsection{Potências fracionárias de operadores setoriais}

Suponhamos que $A$ seja um operador setorial para o qual $\operatorname{Re} \sigma(\mathrm{A})>0$. Então, para todo $\alpha>0$, definimos

$$
A^{-\alpha}=\frac{1}{\Gamma(\alpha)} \int_{0}^{\infty} t^{\alpha-1} e^{-A t} d t
$$

O operador $A^{\alpha}$ é chamado operador de potência fracionária associado ao operador $A$. Definimos $A^{\alpha}$ como sendo a inversa de $A^{-\alpha}$, para $\alpha>0, \operatorname{com} \mathscr{D}\left(A^{\alpha}\right)=R\left(A^{-\alpha}\right)$, e $A^{0}$ como sendo a identidade em $X$.

Se $A: \mathscr{D}(A) \subset X \rightarrow X$ é um operador setorial e $\alpha \geqslant 0$, então o espaço de potência fracionária $X^{\alpha}$ associado a $A$ é definido como $X^{\alpha}=\mathscr{D}\left(A_{1}^{\alpha}\right)$, munido da norma do gráfico $\|x\|_{\alpha}=$ 
$\left\|A_{1}^{\alpha} x\right\|_{X}, x \in X^{\alpha}$, onde $A_{1}=A+a I$ satisfaz $\operatorname{Re} \sigma\left(A_{1}\right)>0$. Observemos que se $\operatorname{Re} \sigma(A)>0$, então podemos ter $X^{\alpha}=\mathscr{D}\left(A^{\alpha}\right)$.

Teorema 1.9. Se A é um operador setorial em $X \operatorname{com} \operatorname{Re} \sigma(\mathrm{A})>0$, então para todo $\alpha>0, A^{-\alpha}$ é um operador linear limitado em $X$, injetivo, e satisfaz $A^{-\alpha} A^{-\beta}=A^{-(\alpha+\beta)}$, quaisquer que sejam $\alpha>0, \beta>0$. Além disso, para $0<\alpha<1$,

$$
A^{-\alpha}=\frac{\operatorname{sen} \pi \alpha}{\pi} \int_{0}^{\infty} \lambda^{-\alpha}(\lambda+A)^{-1} d \lambda .
$$

Os seguintes resultados podem ser encontrados em Henry [15].

Teorema 1.10. Se A é um operador setorial em $X \operatorname{com} \operatorname{Re} \sigma(\mathrm{A})>0$, então $X^{\alpha}$ é um espaço de Banach com a norma $\|\cdot\|_{\alpha}$ para $\alpha \geqslant 0, X^{0}=X$ e, para $\alpha \geqslant \beta \geqslant 0$, temos $X^{\alpha} \hookrightarrow X^{\beta}$. Ademais, se A tem resolvente compacto, então a imersão $X^{\alpha} \hookrightarrow X^{\beta}$ é compacta para $\alpha>\beta \geqslant 0$.

Teorema 1.11. Seja A um operador setorial com $\operatorname{Re} \sigma(\mathrm{A})>\delta>0$. Para todo $\alpha \geqslant 0$, existe uma constante $C_{\alpha}<\infty$ satisfazendo

$$
\left\|A^{\alpha} e^{-A t}\right\|_{\mathscr{L}(X)} \leqslant C_{\alpha} t^{-\alpha} e^{-\delta t}, \quad \text { para } t>0
$$

Lema 1.12 (Desigualdade do Momento). Para quaisquer $\beta<\alpha<\gamma$, se $x \in \mathscr{D}\left(A^{\gamma}\right)$, vale a seguinte desigualdade:

$$
\left\|A^{\alpha} x\right\| \leqslant C(\beta, \alpha, \gamma)\left\|A^{\gamma}\right\|^{\frac{\alpha-\beta}{\gamma-\beta}}\left\|A^{\beta} x\right\|^{\frac{\gamma-\alpha}{\gamma-\beta}}
$$

\subsubsection{Problema de Cauchy abstrato}

Consideramos nesta seção equações não-lineares da forma

$$
\left\{\begin{array}{l}
u_{t}+A u=f(t, u), \quad t>t_{0} \\
u\left(t_{0}\right)=u_{0}
\end{array}\right.
$$

onde $A: \mathscr{D}(A) \subset X \rightarrow X$ é um operador setorial positivo, tal que suas potências fracionárias $A^{\alpha}$ estão bem definidas, e os espaços $X^{\alpha}=\mathscr{D}\left(A^{\alpha}\right)$ com a norma do gráfico $\|x\|_{\alpha}=\left\|A^{\alpha} x\right\|$ estão definidos para $\alpha \geqslant 0$. Assumimos ainda que $f: U \rightarrow X$, em que $U \subset \mathbb{R} \times X^{\alpha}$ é aberto. 
Uma solução de (1.6) é uma função contínua $u:\left[t_{0}, t_{1}\right) \rightarrow X$, diferenciável em $\left(t_{0}, t_{1}\right)$, com $u\left(t_{0}\right)=u_{0}, f(\cdot, u(\cdot)):\left[t_{0}, t_{1}\right) \rightarrow X$ contínua, $u(t) \in \mathscr{D}(A)$, para $t \in\left(t_{0}, t_{1}\right)$, e que satisfaz a equação (1.6).

O teorema seguinte garante a existência de soluções locais para a equação (1.6).

Teorema 1.13. Sejam A um operador setorial, $0 \leqslant \alpha<1, U \subset \mathbb{R} \times X^{\alpha}$, e $f: U \rightarrow X$. Suponhamos que f seja Hölder contínua na variável t e localmente Lipschitz contínua na variável $x$ em $U$, ou seja, suponhamos que para $\left(t_{1}, u_{1}\right) \in U$ exista uma vizinhança $V \subset U$ de $\left(t_{1}, u_{1}\right)$ tal que, para $(t, u),(s, v) \in V$,

$$
\|f(t, u)-f(s, v)\|_{X} \leqslant L\left(|t-s|^{\theta}+\|u-v\|_{\alpha}\right),
$$

sendo $\theta$ e L constantes positivas. Então, qualquer que seja $\left(t_{0}, u_{0}\right) \in U$, existe um instante $\tau=\tau\left(t_{0}, u_{0}\right)>0$ de tal maneira que a equação (1.6) possui uma única solução u definida em $\left(t_{0}, t_{0}+\tau\right)$.

Acerca da existência de soluções globais para a equação (1.6), temos o seguinte resultado:

Teorema 1.14. Suponhamos que as hipóteses sobre A e $f$ enunciadas no Teorema 1.13 estejam satisfeitas, e assumamos também que, para todo conjunto limitado $B \subset U$, a imagem $f(B)$ seja limitada em X. Se u é uma solução de (1.6) em $\left(t_{0}, t_{1}\right)$ e $t_{1}$ é maximal então, ou $t_{1}=\infty$ ou existe uma seqüência $t_{n} \stackrel{n \rightarrow \infty}{\longrightarrow} t_{1}^{-}$de tal maneira que $\left(t_{n}, u\left(t_{n}\right)\right) \rightarrow \partial U$.

\subsection{Teoria geral de atratores}

Sejam $X$ um espaço de Banach e $A: D(A) \subset X \rightarrow X$ um operador setorial com resolvente compacto. Suponhamos que $\operatorname{Re} \sigma(-A)=\{\operatorname{Re} \mu: \mu \in \sigma(-A)\} \subset(-\infty, 0)$. Utilizando a notação de potências fracionárias introduzida na seção anterior, podemos afirmar que existem constantes $M \geqslant 1$ e $\omega>0$ tal que

$$
\left\|A^{\alpha} e^{-A t}\right\|_{\mathscr{L}(X)} \leqslant M t^{-\alpha} e^{-\omega t}, \text { para todo } t>0
$$

Estudaremos nesta seção a existência de atratores globais para o problema parabólico

$$
\left\{\begin{array}{l}
\dot{u}+A u=f(u) \\
u(0)=u_{0} \in X^{\alpha}
\end{array}\right.
$$


em que o operador $A$ satisfaz as condições dadas acima e $f: X^{\alpha} \rightarrow X$ é globalmente Lipschitz e globalmente limitada. Também é nosso objetivo estudar a continuidade dos atratores correspondentes a uma família de semigrupos. A principal referência bibliográfica desta seção é Hale [14].

Segue do que fizemos na subseção anterior que as soluções para o problema (1.9) estão globalmente definidas. Se $u\left(t, u_{0}\right)$ denota uma solução de (1.9), podemos definir um $C_{0}$-semigrupo não-linear $T(t): X^{\alpha} \rightarrow X^{\alpha}$ através da relação

$$
T(t) u_{0}:=u\left(t, u_{0}\right)
$$

de modo que o semigrupo em questão seja dado pela Fórmula da Variação das Constantes

$$
T(t) u_{0}=e^{-A t} u_{0}+\int_{0}^{t} e^{-A(t-s)} f\left(T(s) u_{0}\right) d s
$$

\subsubsection{Existência e limitação}

Nesta subseção enunciamos importantes resultados que conduzem à existência e limitação de atratores para o semigrupo gerado pela equação (1.9).

Para provarmos a existência de atratores, introduzimos a seguir algumas definições necessárias e também alguns lemas úteis.

Para cada $u_{0} \in X^{\alpha}$, a órbita positiva $\gamma^{+}\left(u_{0}\right)$ por $u_{0}$ é definida como $\gamma^{+}\left(u_{0}\right)=\left\{T(t) u_{0}: t \geqslant\right.$ $0\}$. Uma órbita negativa por $u_{0}$ é uma função contínua $\phi:(-\infty, 0] \rightarrow X^{\alpha}$ tal que $\phi(0)=u_{0}$ e, para todo $s \leqslant 0, T(t) \phi(s)=\phi(t+s)$, para $0 \leqslant t \leqslant-s$. Uma órbita completa por $u_{0}$ é uma função $\phi: \mathbb{R} \rightarrow X^{\alpha}$ tal que $\phi(0)=u_{0}$ e que, para todo $s \in \mathbb{R}$, satisfaz $T(t) \phi(s)=\phi(t+s)$, para $t \geqslant 0$.

Como a imagem $T(t)$ pode não ser todo o espaço $X^{\alpha}$, dizer que existe uma órbita negativa ou uma órbita completa impõe certas restrições sobre $u_{0}$. Além disso, como $T(t)$ em geral não é injetivo, uma órbita negativa (se existe uma) pode não ser única. Seja a órbita negativa por $u_{0}$ definida como a união de todas as órbitas negativas por $u_{0}$. Então

$$
\begin{gathered}
\gamma^{-}\left(u_{0}\right)=\bigcup_{t \geqslant 0} H\left(t, u_{0}\right) \\
H\left(t, u_{0}\right)=\left\{\begin{array}{c}
\left\{y \in X^{\alpha}: \text { existe uma órbita negativa } u_{0}\right. \text { definida por } \\
\left.\phi:(-\infty, 0] \rightarrow X^{\alpha} \operatorname{com} \phi(0)=u_{0} \text { e } \phi(-t)=y\right\}
\end{array}\right.
\end{gathered}
$$


Uma órbita completa $\gamma\left(u_{0}\right)$ por $u_{0}$ é definida como $\gamma\left(u_{0}\right)=\gamma^{+}\left(u_{0}\right) \cup \gamma^{-}\left(u_{0}\right)$. Para todo $B \subset X^{\alpha}$, sejam $\gamma^{+}(B)=\bigcup_{u_{0} \in B} \gamma^{+}\left(u_{0}\right), \gamma^{-}(B)=\bigcup_{u_{0} \in B} \gamma^{-}\left(u_{0}\right), \gamma(B)=\bigcup_{u_{0} \in B} \gamma\left(u_{0}\right)$, respectivamente, as órbitas positiva, negativa e completa por $B$ quando existem.

Para todo conjunto $B \subset X^{\alpha}$, definimos o conjunto $\omega$-limite $\omega(B)$ de $B$ e o conjunto $\alpha$-limite $\alpha(B)$ de $B$ como

$$
\omega(B)=\bigcap_{s \geqslant 0} \overline{\bigcup_{t \geqslant s} T(t) B}, \quad \alpha(B)=\bigcap_{s \geqslant 0} \overline{\bigcup_{t \geqslant s} H(t, B)} .
$$

Dizemos que um conjunto $B \subset X^{\alpha}$ atrai um conjunto $C$ sob $T(t)$ se $\operatorname{dist}(T(t) C, B) \rightarrow 0$ quando $t \rightarrow \infty$. Um conjunto $S \subset X^{\alpha}$ é invariante se, para $u_{0} \in S$, existe uma órbita completa $\gamma\left(u_{0}\right)$ por $u_{0}$ de tal forma que $\gamma\left(u_{0}\right) \subset S$.

Um conjunto $\mathscr{A} \subset X^{\alpha}$ é um atrator global para $\{T(t): t \geqslant 0\}$ se é um conjunto compacto, invariante e atrai subconjuntos limitados de $X^{\alpha}$.

Lema 1.15. Um conjunto $S \subset X^{\alpha}$ é invariante se, e somente se, $T(t) S=S$, para todo $t \geqslant 0$.

Demonstração. Suponhamos primeiramente que $S$ seja invariante. Então, para todo $x \in S$, existe uma órbita completa $\gamma(x)$ de $x$ tal que $\gamma(x) \subset S$. Em particular, existe uma órbita negativa $\phi:(-\infty, 0] \rightarrow H^{1}(\Omega)$ de $x$. Fixando $t_{0} \geqslant 0$ e tomando $y=\phi\left(-t_{0}\right)$, temos $y \in S$ e $T\left(t_{0}\right) y=x$. Logo, $S \subset T(t) S$, para todo $t \geqslant 0$. Por outro lado, se $x \in S$, então $\gamma^{+}(x)=\{T(t) x: t \geqslant 0\} \subset S$, ou seja, $T(t) S \subset S$, qualquer que seja $t \geqslant 0$. Conseqüentemente, $T(t) S=S$, para todo $t \geqslant 0$.

Suponhamos agora que $T(t) S=S$, para todo $t \geqslant 0$. Se $x \in S$, existe $y_{1} \in S$ tal que $T(1) y_{1}=$ $x:=y_{0}$. Do mesmo modo, existe $y_{2} \in S$ tal que $T(2) y_{2}=y_{1}$. Prosseguindo indutivamente, obtemos $T(1) y_{n}=y_{n-1}$, para todo $n \geqslant 1$. Observemos ainda que $T(k) y_{n}=y_{n-k}$, para todo $k \leqslant n$. Seja $s \in(-\infty, 0]$, e escolhamos $n \in \mathbb{N}$ tal que $s \in(-n,-n+1]$.

Definamos $\phi:(-\infty, 0] \rightarrow X^{\alpha}$ por $\phi(s)=T(s+n) y_{n}$. Então $\phi(s) \in S$, para todo $s \leqslant 0$, $\phi(0)=T(0+1) y_{1}=x$ e, para quaisquer $0 \leqslant t \leqslant-s, s<0, s \in(-n,-n+1]$, temos $T(t) \phi(s)=$ $T(t) T(s+n) y_{n}=T(t+s+n) y_{n}$. Por outro lado, se $0 \leqslant t \leqslant-s$, então $t+s \leqslant 0$ e $t+s \in$ $(-j,-j+1]$, para algum $j \leqslant n$. Logo, $\phi(t+s)=T(t+s+j) y_{j}$. Mas

$$
\begin{aligned}
T(t) \phi(s) & =T(t+s+n) y_{n}=T(t+s+j+n-j) y_{n}=T(t+s+j) T_{0}(n-j) y_{n} \\
& =T(t+s+j) y_{n-(n-j)}=T(t+s+j) y_{j}=\phi(t+s) .
\end{aligned}
$$

Desta forma, a função $\phi$ definida acima é contínua, e então, definindo $\bar{\phi}: \mathbb{R} \rightarrow X^{\alpha}$ por

$$
\bar{\phi}(s)=\left\{\begin{aligned}
T(s) x, & s>0 \\
\phi(s), & s \leqslant 0
\end{aligned}\right.
$$


segue que $\bar{\phi}$ é uma órbita completa de $x$ que está inteiramente contida em $S$. Portanto, $S$ é invariante.

Lema 1.16. Se $S \subset X^{\alpha}$ é invariante sob $\{T(t): t \geqslant 0\}$ e $v \in S$, então existe uma órbita completa de v.

Lema 1.17. Um ponto $v \in \omega(B)$ se, e somente se, existem uma seqüência $t_{n} \rightarrow+\infty$ e $v_{n} \in B$ tais que $T\left(t_{n}\right) v_{n} \rightarrow v$.

Lema 1.18. Um ponto $v \in \alpha(B)$ se, e somente se, existem seqüências $t_{n} \rightarrow+\infty$ e $v_{n} \in B$ tais que cada $v_{n}$ possui uma única órbita negativa $\phi_{n}:(-\infty, 0] \rightarrow X^{\alpha}\left(\phi_{n}(0)=v_{n}\right)$ e $\phi_{n}\left(-t_{n}\right) v_{n} \rightarrow v$. $\diamond$

Lema 1.19. Para todo conjunto limitado $B \subset X^{\alpha}$, o conjunto $\gamma^{+}(B)$ é limitado e $T(t) \gamma^{+}(B)$ é compacto, para todo $t>0$.

Demonstração. Como a imersão $X^{\alpha} \hookrightarrow X^{\beta}$ é compacta para $\alpha>\beta \geqslant 0$ e $T(t): X^{\alpha} \rightarrow X^{\beta}$ é limitado, segue que $T(t): X^{\alpha} \rightarrow X^{\alpha}$ é compacto. Usando a Fórmula da Variação das Constantes, as estimativas usuais e as hipóteses sobre $B$ e $f$, segue que $\gamma^{+}(B)$ é limitado em $X^{\alpha}$ sempre que $B$ é limitado em $X^{\alpha}$. Logo, como $\gamma^{+}(B)$ é limitado, temos que $\{T(s) B: s \geqslant t\}=T(t) \gamma^{+}(B)$ é compacto, e o resultado segue.

Lema 1.20. Para todo $u_{0} \in X^{\alpha}, \omega\left(u_{0}\right)$ é não-vazio, conexo, compacto, invariante e atrai $u_{0}$. $\diamond$

Lema 1.21. Seja $u_{0} \in X^{\alpha}$ seja tal que existe uma órbita negativa $\phi:(-\infty, 0] \rightarrow X^{\alpha}$ de $u_{0}$ e tal que $\overline{\phi((-\infty, 0])}$ seja compacto. Definamos

$$
\alpha_{\phi}\left(u_{0}\right)=\left\{v \in X^{\alpha}: \text { existe } t_{n} \rightarrow \infty \text { tal que } \phi\left(-t_{n}\right) \rightarrow v\right\} .
$$

Então $\alpha_{\phi}\left(u_{0}\right)$ é não-vazio, conexo, compacto e invariante.

Demonstração. Notemos inicialmente que $\alpha_{\phi}\left(u_{0}\right)=\bigcap_{t \geqslant 0} \overline{\phi((-\infty, t])}$, e deste fato segue claramente que $\alpha_{\phi}\left(u_{0}\right)$ é não-vazio, conexo e compacto. Mostremos agora que $\alpha_{\phi}\left(u_{0}\right)$ é invariante. De fato, se $v \in \alpha_{\phi}\left(u_{0}\right)$, então existe uma seqüência $t_{n} \rightarrow+\infty$ tal que $\phi\left(-t_{n}\right) \rightarrow v$. Da continuidade de $T(t): X^{\alpha} \rightarrow X^{\alpha}$, segue que $T(t) \phi\left(-t_{n}\right)=\phi\left(t-t_{n}\right) \rightarrow T(t) v$, e então $T(t) v \in$ $\alpha_{\phi}\left(u_{0}\right)$. Por outro lado, se $w \in \alpha_{\phi}\left(u_{0}\right)$, existe uma seqüência $t_{n} \rightarrow \infty$ (sem perda de generalidade, assumiremos que $\left.t_{n} \geqslant t, n \in \mathbb{N}\right)$ tal que $\phi\left(-t_{n}\right) \rightarrow w$. Como $\left\{\phi\left(-t_{n}-t\right): n \in \mathbb{N}\right\}$ é relativamente compacto, tomando subseqüências se necessário, existe $z \in X^{\alpha}$ tal que $\phi\left(-t_{n}-t\right) \rightarrow z$ e $z \in \alpha_{\phi}\left(u_{0}\right)$. Da unicidade do limite, segue que $T(t) z=w$. 
Lema 1.22. Existe constante $N>0$ tal que, para cada conjunto limitado $B \subset X^{\alpha}$, existe um instante $\tau_{B}>0$ de tal forma que

$$
\sup _{t \geqslant \tau_{B}} \sup _{w \in T(t) B}\|w\|_{X^{\alpha}} \leqslant N
$$

Além disso,

$$
\sup _{\substack{B \subset X^{\alpha} \\ B \text { limitado }}} \sup _{v \in \omega(B)}\|v\|_{X^{\alpha}} \leqslant N .
$$

Demonstração. Dados um conjunto limitado $B \subset X^{\alpha}$ e $v \in B$, segue de (1.10) que

$$
T(t) v=e^{-A_{0} t} v+\int_{0}^{t} e^{-A_{0}(t-s)} f(T(s) v) d s
$$

Das hipóteses sobre $f$ e $A$, segue a existência de uma constante $K$ tal que $\|f(w)\|_{X} \leqslant K$, para todo $w \in X^{\alpha} \mathrm{e}$

$$
\|T(t) v\|_{X^{\alpha}} \leqslant M e^{-\beta t}\|v\|_{X^{\alpha}}+M K \int_{0}^{t}(t-s)^{-\alpha} e^{-\beta(t-s)} d s
$$

Como $v$ está em um subconjunto limitado de $X^{\alpha}$, existe $\tau_{B}$ tal que

$$
\|T(t) v\|_{X^{\alpha}} \leqslant 1+M K \beta^{-\alpha} \Gamma(1-\alpha)=: N, \quad t \geqslant \tau_{B}
$$

Sendo $N$ independente de $B \subset X^{\alpha}$, a demonstração está concluída.

Lema 1.23. Se $B \subset X^{\alpha}$ é um conjunto limitado, então $\omega(B)$ é não-vazio, compacto, invariante e atrai $B$ sob $\{T(t): t \geqslant 0\}$. Além disso, se B é conexo, então $\omega(B)$ é conexo.

Teorema 1.24. Se $B_{N}=\left\{u \in X^{\alpha}:\|u\|_{X^{\alpha}} \leqslant N\right\}$, então $\omega\left(B_{N}\right)$ é o atrator global para $\{T(t)$ : $t \geqslant 0\}$.

Demonstração. Claramente, $\omega(B)$ é compacto, invariante, conexo e atrai $B_{N}$. Notemos primeiramente que para todo conjunto limitado $B \subset X^{\alpha}$, existe $\tau_{B}$ tal que $T(t) B \subset B_{N}$, para todo $t \geqslant \tau_{B}$. Como $B_{N}$ é atraído por $\omega\left(B_{N}\right)$ e $B_{N}$ atrai $B$, o resultado segue.

Deste resultado e do lema 1.22 segue que

Corolário 1.25. O atrator $\omega\left(B_{N}\right)$ do semigrupo $\{T(t): t \geqslant 0\}$ é limitado.

Lema 1.26. Para todo conjunto limitado $B \subset X^{\alpha}$, para o qual o conjunto $\overline{\gamma^{-}(B)}$ é não-vazio $e$ compacto, o conjunto, $\alpha(B)$ é não-vazio, compacto e invariante. 


\subsubsection{Caracterização de atratores para sistemas gradientes}

Dizemos que o semigrupo $\{T(t): t \geqslant 0\}$ tem estrutura gradiente se existe uma função contínua $V: X^{\alpha} \rightarrow \mathbb{R}$ que satisfaz

(i) $\mathbb{R}^{+} \ni t \mapsto V\left(T(t) u_{0}\right) \in \mathbb{R}$ é não-crescente

(ii) $\mathscr{E}=\left\{u \in X^{\alpha}: A u+f(u)=0\right\}=\left\{u_{0} \in X^{\alpha}: V\left(T(t) u_{0}\right)=V\left(u_{0}\right)\right.$, para todo $\left.t \in \mathbb{R}\right\}$.

Definimos a variedade estável de $u_{0}^{*} \in \mathscr{E}$ como

$$
\mathrm{W}^{s}\left(u_{0}^{*}\right)=\left\{u_{0} \in X: u\left(t, u_{0}\right) \rightarrow u_{0}^{*} \text { quando } t \rightarrow \infty\right\}
$$

e o variedade instável de $u_{0}^{*} \in \mathscr{E}$ como

$\mathrm{W}^{u}\left(u_{0}^{*}\right)=\left\{u_{0} \in X: u\left(t, u_{0}\right)\right.$ está definido para todo $t \leqslant 0$ e $u\left(t, u_{0}\right) \rightarrow u_{0}^{*}$ quando $\left.t \rightarrow-\infty\right\}$.

Mostramos nesta seção que se o semigrupo $\{T(t): t \geqslant 0\}$ tem estrutura gradiente e o conjunto $\mathscr{E}$ consiste apenas de pontos isolados, então o atrator $\mathscr{A}$ pode ser escrito como a união das variedades instáveis dos elementos de $\mathscr{E}$.

Lema 1.27. Se todos os pontos de $\mathscr{E}$ são isolados, então para todo $u_{0} \in X^{\alpha}$, temos que $\omega\left(u_{0}\right)=$ $\left\{u_{0}^{*}\right\}$, para algum $u_{0}^{*} \in \mathscr{E}$. Como conseqüência deste fato, $T(t) u_{0} \rightarrow u_{0}^{*}$ quando $t \rightarrow \infty$.

Demonstração. Notemos inicialmente que se $v \in \omega\left(u_{0}\right)$, então existe uma seqüência $t_{n} \rightarrow \infty$ tal que $T\left(t_{n}\right) u_{0} \rightarrow v$. Como $V\left(T(t) u_{0}\right)$ é decrescente e limitada inferiormente, existe um $\ell \in \mathbb{R}$ tal que $V\left(T(t) u_{0}\right) \rightarrow \ell$ quando $t \rightarrow \infty$. Além disso, como $T(t) T\left(t_{n}\right) u_{0} \rightarrow T(t) v$ e $V$ é contínua, temos que $V(T(t) v)=\ell$, para todo $t \in \mathbb{R}$. Assim, como $V\left(T\left(t_{n}\right) u_{0}\right) \rightarrow V(v)$, segue que $V(v)=$ $\ell=V(T(t) v)$ e $v \in \mathscr{E}$. Como o conjunto $\mathscr{E}$ consiste de apenas um número finito de pontos e $\omega\left(u_{0}\right)$ é conexo, segue que $\omega\left(u_{0}\right)=\left\{u_{0}^{*}\right\}$, para algum $u_{0}^{*} \in \mathscr{E}$.

O próximo resultado garante que se existe uma órbita negativa de $u_{0}$ que é limitada, então $u_{0}$ está na variedade invariante de algum $u_{0}^{*} \in \mathscr{E}$.

Lema 1.28. Suponhamos que $u_{0} \in X^{\alpha}$ seja tal que existe uma órbita negativa $\phi:(-\infty, 0] \rightarrow X^{\alpha}$ de $u_{0}$ e que $\overline{\phi((-\infty, 0])}$ seja compacto. Se $\mathscr{E}$ possui apenas pontos isolados, então existe $u_{0}^{*} \in \mathscr{E}$ de tal forma que $\alpha_{\phi}\left(u_{0}\right)=\left\{u_{0}^{*}\right\}$. Como conseqüência deste fato, temos que $\phi(t) \rightarrow u_{0}^{*}$ quando $t \rightarrow-\infty$. 
Demonstração. Notemos inicialmente que se $v \in \alpha_{\phi}\left(u_{0}\right)$, então existe uma seqüência $t_{n} \rightarrow \infty$ tal que $\phi\left(-t_{n}\right) \rightarrow v$. Como $V(\phi(-t))$ é crescente e limitada superiormente, existe um $r \in \mathbb{R}$ de tal modo que $V(\phi(-t)) \rightarrow r$ quando $t \rightarrow \infty$. Como anteriormente, temos $V(T(t) v)=r$, para todo $t \in \mathbb{R}$, e $v \in \mathscr{E}$. Como o conjunto $\mathscr{E}$ consiste de apenas um número finito de pontos e $\alpha_{\phi}\left(u_{0}\right)$ é conexo, resulta que $\alpha_{\phi}\left(u_{0}\right)=\left\{u_{0}^{*}\right\}$, para algum $u_{0}^{*} \in \mathscr{E}$. Além disso, para qualquer seqüência $t_{n} \rightarrow \infty,\left\{\phi\left(-t_{n}\right)\right\}$ possui uma subseqüência que converge para $u_{0}^{*}$, e portanto $\phi(t) \rightarrow u_{0}^{*}$ quando $t \rightarrow-\infty$.

Lema 1.29. Seja S um subconjunto relativamente compacto de $X^{\alpha}$, invariante $\operatorname{sob}\{T(t): t \geqslant 0\}$. Se $v \in S$, então existem $v^{+}, v^{-} \in \mathscr{E}$ tais que $v \in \mathrm{W}^{u}\left(v^{+}\right)$e $v \in \mathrm{W}^{s}\left(v^{-}\right)$.

Demonstração. Seja $v \in S$. Como $S$ é invariante, existe uma órbita completa $\mathbb{R} \ni t \mapsto T(t) v \in S$ de $v$. Da compacidade de $\bar{S}$, segue que esta órbita completa é compacta. Do Lema 1.27, existe $u_{1}^{*} \in \mathscr{E}_{0}$ tal que $T(t) v \rightarrow u_{1}^{*}$. Da mesma forma, do Lema 1.28, existe $u_{2}^{*} \in S$ tal que $\phi(t) v \rightarrow u_{2}^{*}$ quando $t \rightarrow-\infty$. Fazendo $v^{-}=u_{1}^{*}$, e $v^{+}=u_{2}^{*}$, segue o resultado.

Deste lema resulta o seguinte resultado:

Teorema 1.30. Seja $\mathscr{A}$ o atrator para $\{T(t): t \geqslant 0\}$. Então $\mathscr{A}=\bigcup_{u_{0}^{*} \in \mathscr{E}} \mathrm{W}^{u}\left(u_{0}^{*}\right)$.

\subsubsection{Continuidade de atratores}

Sejam $X$ um espaço de Banach, $\Lambda$ um espaço topológico e $J_{\lambda} \subset X$, para cada $\lambda \in \Lambda$. Denotemos por $d(\cdot, \cdot): X \times X \rightarrow \mathbb{R}^{+}$a métrica induzida pela norma de $X$. Dizemos que:

(a) a família $\left\{J_{\lambda}: \lambda \in \Lambda\right\}$ é semicontínua superiormente em $\lambda=\lambda_{0}$ se $\sup _{u_{\lambda} \in J_{\lambda}} d\left(u_{\lambda}, J_{\lambda_{0}}\right) \stackrel{\lambda \rightarrow \lambda_{0}}{\longrightarrow} 0$.

(b) a família $\left\{J_{\lambda}: \lambda \in \Lambda\right\}$ é semicontínua inferiormente em $\lambda=\lambda_{0}$ se $\sup _{u \in J_{\lambda_{0}}} d\left(u, J_{\lambda}\right) \stackrel{\lambda \rightarrow \lambda_{0}}{\longrightarrow} 0$.

O lema seguinte fornece uma definição alternativa de semicontinuidade superior e inferior.

Lema 1.31. (i) A família $\left\{J_{\lambda}: \lambda \in \Lambda\right\}$ é semicontínua superiormente em $\lambda=\lambda_{0}$ se toda seqüência $\left\{u_{\lambda_{n}}\right\}$, com $u_{\lambda_{n}} \in J_{\lambda_{n}}, n \in \mathbb{N}, \lambda_{n} \rightarrow \lambda_{0}$, possui uma subseqüência convergente para um elemento de $J_{\lambda_{0}}$.

(ii) Se $J_{\lambda_{0}}$ é compacto e para todo $u \in J_{\lambda_{0}}$, existe uma seqüência $\left\{u_{\lambda_{n}}\right\}$ com $u_{\lambda_{n}} \in J_{\lambda_{n}}, n \in$ $\mathbb{N}, \lambda_{n} \rightarrow \lambda_{0}$, tal que $u_{\lambda_{n}} \rightarrow u$, então $\left\{J_{\lambda}: \lambda \in \Lambda\right\}$ é semicontínua inferiormente em $\lambda=\lambda_{0}$. 
Demonstração. Provemos primeiramente o item (i). Para tanto suponhamos, por absurdo, que a família $\left\{J_{\lambda}: \lambda \in \Lambda\right\}$ não seja semicontínua superiormente em $\lambda=\lambda_{0}$. Então existem $\eta>0$ e $\lambda_{n} \rightarrow \lambda_{0}$ tais que $\sup d\left(u_{\lambda_{n}}, J_{\lambda_{0}}\right)>\eta$. Desta forma, para cada $n \in \mathbb{N}$, existe $\bar{u}_{n} \in J_{\lambda_{n}}$ tal que $d\left(\bar{u}_{\lambda_{n}}, J_{\lambda_{0}}\right)>\frac{\eta}{2}$. Agora, por hipótese, a seqüência $\left\{\bar{u}_{\lambda_{n}}\right\}$ admite uma subseqüiência convergente, que denotaremos também por $\left\{\bar{u}_{\lambda_{n}}\right\}$, para, digamos, $\bar{u}_{0} \in J_{\lambda_{0}}$ quando $\lambda_{n} \rightarrow \lambda_{0}$. Por outro lado,

$$
d\left(\bar{u}_{\lambda_{n}}, \bar{u}_{0}\right)>d\left(\bar{u}_{\lambda_{n}}, J_{\lambda_{0}}\right)>\frac{\eta}{2}
$$

para cada $n \in \mathbb{N}$, uma contradição.

Para provar (ii), suponhamos que a família $\left\{J_{\lambda}: \lambda \in \Lambda\right\}$ não seja semicontínua inferiormente em $\lambda=\lambda_{0}$. Então existem $\eta>0$ e $\lambda_{n} \rightarrow \lambda_{0}$ tais que $\sup _{u \in J_{\lambda_{0}}} d\left(u, J_{\lambda_{n}}\right)>\eta$. Como $J_{\lambda_{0}}$ é compacto, existe $u_{0}=u_{0}(n) \in J_{\lambda_{0}}$ tal que $d\left(u_{0}, J_{\lambda_{n}}\right)=\sup _{u \in J_{\lambda_{0}}} d\left(u, J_{\lambda_{n}}\right)$, para cada $n \in \mathbb{N}$. Além disso, existem uma subseqüência de $u_{0}(n)$, que denotaremos da mesma forma, e $\bar{u}_{0} \in J_{\lambda_{0}}$ de tal modo que $u_{0}(n) \rightarrow \bar{u}_{0}$ quando $n \rightarrow \infty$. Por hipótese, existe uma seqüência $\left\{u_{n}\right\}, \operatorname{com} u_{n} \in J_{\lambda_{n}}, n \in \mathbb{N}$, tal que $u_{n} \rightarrow \bar{u}_{0}$, ou seja, $d\left(u_{n}, \bar{u}_{0}\right) \rightarrow 0$ quando $n \rightarrow \infty$. Mas, para cada $n \in \mathbb{N}$,

$$
d\left(\bar{u}_{0}, u_{n}\right)>d\left(\bar{u}_{0}, J_{\lambda_{n}}\right)=\sup _{u \in J_{\lambda_{0}}} d\left(u, J_{\lambda_{n}}\right)>\eta
$$

contradizendo o fato de que $u_{n} \rightarrow \bar{u}_{0}$ quando $\lambda_{n} \rightarrow \lambda_{0}$.

Seja $\left\{T_{\lambda}(t): t \geqslant 0\right\}$ uma família de semigrupos tais que, para cada $\lambda \in \Lambda$, o semigrupo $\left\{T_{\lambda}(t): t \geqslant 0\right\}$ possua um atrator global $\mathscr{A}_{\lambda}$.

O seguinte teorema garante que, se a união dos atratores é relativamente compacta em $X$ e a família de semigrupos $\left\{T_{\lambda}(t): \lambda \in \Lambda, t \geqslant 0\right\}$ é contínua com relação ao parâmetro $\lambda$, então a família de atratores $\left\{\mathscr{A}_{\lambda}\right\}$ é semicontínua superiormente.

Teorema 1.32. Suponhamos que o conjunto $\cup\left\{\mathscr{A}_{\lambda}: \lambda \in \Lambda\right\}$ seja relativamente compacto em $X$ e que para $u_{\lambda} \stackrel{\lambda \rightarrow \lambda_{0}}{\longrightarrow} u_{\lambda_{0}}$ em $X$, nós tenhamos $\left\|T_{\lambda}(t) u_{\lambda}-T_{\lambda_{0}}(t) u_{\lambda_{0}}\right\| \rightarrow 0$ quando $\lambda \rightarrow \lambda_{0}$, para todo $t \in \mathbb{R}^{+}$. Então a família de atratores $\left\{\mathscr{A}_{\lambda}: \lambda \in \Lambda\right\}$ é semicontínua superiormente em $\lambda=\lambda_{0}$

Demonstração. Se $\left\{u_{\lambda_{n}}\right\}$ é uma seqüência tal que $u_{\lambda_{n}} \in \mathscr{A}_{\lambda_{n}}, n \in \mathbb{N}$, segue da compacidade do conjunto $\cup\left\{\mathscr{A}_{\lambda}: \lambda \in \Lambda\right\}$ que existe $u_{\lambda_{0}} \in X$, com $u_{\lambda_{n}} \rightarrow u_{\lambda_{0}}$ quando $\lambda_{n} \rightarrow \lambda_{0}$. Para mostrarmos que $u_{\lambda_{0}} \in \mathscr{A}_{\lambda_{0}}$, basta provarmos que existe uma solução completa limitada que passa por $u_{\lambda_{0}}$. 
Para cada $n \in \mathbb{N}$, temos uma órbita completa limitada

$$
\begin{aligned}
\phi_{n} & : \mathbb{R} \rightarrow X \\
t & \mapsto \phi_{n}\left(t, u_{\lambda_{n}}\right)
\end{aligned}
$$

por $u_{\lambda_{n}}$. Para $t \geqslant 0$, segue da continuidade dos semigrupos não-lineares que

$$
\phi_{n}\left(t, u_{\lambda_{n}}\right)=T_{\lambda_{n}}\left(t, u_{\lambda_{n}}\right) \stackrel{\lambda_{n} \rightarrow \lambda_{0}}{\longrightarrow} T_{\lambda_{0}}\left(t, u_{\lambda_{0}}\right)
$$

Para $t<0$, construímos a órbita por $u_{\lambda_{0}}$ do seguinte modo: para $t \in(-k,-k+1], k \in \mathbb{Z}^{+}$, consideramos a seqüência $\left\{\phi_{n}\left(-k, u_{\lambda_{n}}\right)\right\}_{n \in \mathbb{N}}$ em $\overline{\bigcup_{\lambda \in \Lambda} \mathscr{A}_{\lambda}}$. Seguindo o raciocínio que anteriormente foi usado para a seqüência $\left\{u_{\lambda_{n}}\right\}$, obtemos que $\phi_{n}\left(-k, u_{\lambda_{n}}\right) \rightarrow \psi_{0}\left(-k, u_{\lambda_{0}}\right)$. Portanto,

$$
\begin{aligned}
\phi_{n}\left(t, u_{\lambda_{n}}\right) & =\phi_{n}\left(t+k, \phi_{n}\left(-k, u_{\lambda_{n}}\right)\right) \\
& =T_{\lambda_{n}}\left(t+k, \phi_{n}\left(-k, u_{\lambda_{n}}\right)\right) \rightarrow T_{\lambda_{0}}\left(t+k, \psi_{0}\left(-k, u_{\lambda_{0}}\right)\right):=\tilde{\phi}_{0}(t, u) .
\end{aligned}
$$

Finalmente, definindo

$$
\phi_{0}\left(t, u_{\lambda_{0}}\right)= \begin{cases}T_{\lambda_{0}}\left(t, u_{\lambda_{0}}\right), & \text { para } t \geqslant 0 \\ \tilde{\phi}_{0}\left(t, u_{\lambda_{0}}\right), & \text { para } t<0\end{cases}
$$

segue que $\phi_{0}\left(t, u_{\lambda_{0}}\right)$ é uma órbita completa limitada por $u_{\lambda_{0}}$. Portanto, $u_{\lambda_{0}} \in \mathscr{A}_{\lambda_{0}}$.

\subsection{Teoremas de imersões de Sobolev}

De uma forma geral, as propriedades de imersão de Espaços de Sobolev dependem das propriedades de regularidade do domínio $\Omega$. Os resultados desta seção, bem como suas demonstrações, podem ser encontrados em Adams [1] e Brezis [7].

Dados um ponto $x \in \mathbb{R}^{N}$, uma bola aberta $B_{1}$ com centro em $x$, e uma bola aberta $B_{2}$ que não contém $x$, o conjunto $C_{x}=B_{1} \cap\left\{x+\lambda(y-x): y \in B_{2}, \lambda>0\right\}$ é um cone finito em $\mathbb{R}^{N}$ com vértice em $x$.

Dizemos que um domínio $\Omega \subset \mathbb{R}^{N}$ tem a propriedade do cone se existe um cone finito $C$ de tal forma que cada ponto $x \in \Omega$ é o vértice de um cone finito $C_{x}$ contido em $\Omega$ e congruente a C.

Teorema 1.33 (Rellich-Kondrachov). Suponhamos que $\Omega \subset \mathbb{R}^{N}$ seja limitado e de classe $\mathscr{C}^{1}$. Então 
(i) $W^{1, p}(\Omega) \subset L^{q}(\Omega)$, para todo $q \in\left[1, p^{*}\right)$, onde $\frac{1}{p^{*}}=\frac{1}{p}-\frac{1}{N}$ e $p<N$,

(ii) $W^{1, p}(\Omega) \subset L^{q}(\Omega)$, para todo $q \in[1, \infty)$ e $p=N$,

(iii) $W^{1, p}(\Omega) \subset C(\bar{\Omega})$, onde $p>N$,

com imersões compactas.

Teorema 1.34. Suponhamos que $\Omega$ seja um domínio que tenha a propriedade do cone em $\mathbb{R}^{N}$, e sejam $s>0$ e $1<p<N$. Então as seguintes imersões são contínuas:

(i) Se $N>$ sp, então $W^{s, p} \rightarrow L^{r}(\Omega)$, para $p \leqslant r \leqslant \frac{N p}{N-s p}$.

(ii) Se $N=s p$, então $W^{s, p} \rightarrow L^{r}(\Omega)$, para $p \leqslant r<\infty$.

(iii) Se $N<(s-j)$ p par algum inteiro não-negativo $j$, então $W^{s, p} \rightarrow C_{B}^{j}(\Omega)$.

Se $m \geqslant 1$ e $1 \leqslant p<\infty$, então

(iv) $W^{m, p}(\Omega) \subset L^{p^{*}}(\Omega)$, onde $\frac{1}{p^{*}}=\frac{1}{p}-\frac{1}{N} e 1 \leqslant p<N$,

(v) $W^{1, p}(\Omega) \subset L^{q}(\Omega)$, para todo $q \in[p, \infty)$ e $p=N$,

(vi) $W^{1, p}(\Omega) \subset L^{\infty}(\Omega)$, onde $p>N$,

com imersões contínuas.

\subsubsection{Teorema do Traço}

A caracterização dos traços em $\partial \Omega$ de funções definidas em $W^{m, p}(\Omega)$ têm importantes aplicações no estudo de problemas não-homogêneos com condições de fronteira não-lineares para operadores diferenciais definidos em $\Omega$.

Um domínio $\Omega$ de $\mathbb{R}^{N}$ possui a propriedade de $C^{m}$-regularidade uniforme se existem uma cobertura aberta, enumerável e localmente finita $\left\{U_{j}\right\}_{j \in \mathbb{N}}$ de sua fronteira $\partial \Omega$, e uma seqüência $\left\{\phi_{j}\right\}_{j \in \mathbb{N}}$ de transformações $m$-suaves e injetoras, com $\phi_{j}: U_{j} \rightarrow B_{\mathbb{R}^{N}}(0,1)$, de tal maneira que

(i) existe $\delta>0$ tal que $\bigcup_{j=1}^{\infty} \phi_{j}^{-1}\left(B_{\mathbb{R}^{N}}\left(0, \frac{1}{2}\right)\right) \supset \Omega_{\delta}$, onde $\Omega_{\delta}=\{x \in \Omega: d(x, \partial \Omega)<\delta\}$;

(ii) existe um inteiro positivo $m$ tal que toda coleção contendo $m+1$ conjuntos da cobertura $\left\{U_{j}\right\}_{j \in \mathbb{N}}$ possui intersecção vazia; 
(iii) para cada $j, \phi_{j}\left(U_{j} \cap \Omega\right)=\left\{x \in B_{\mathbb{R}^{N}}(0,1): x_{N}>0\right\}$, onde $x=\left(x_{1}, x_{2}, \cdots, x_{N}\right)$;

(iv) se $\left(\phi_{j, 1}, \phi_{j, 2}, \cdots, \phi_{j, n}\right)$ e $\left(\psi_{j, 1}, \psi_{j, 2}, \cdots, \psi_{j, N}\right)$ denotam as componentes de $\phi_{j}$ e $\psi_{j}=\phi_{j}^{-1}$ respectivamente, então existe uma constante positiva $M$ de tal modo que, para todo multiíndice $\alpha, \operatorname{com}|\alpha| \leqslant m$, e $1 \leqslant i, j \leqslant N$,

$$
\begin{aligned}
\left|D^{\alpha} \phi_{j, i}(x)\right| & \leqslant M, \quad x \in U_{j} \\
\left|D^{\alpha} \psi_{j, i}(x)\right| & \leqslant M, \quad x \in B_{\mathbb{R}^{N}}(0,1)
\end{aligned}
$$

Para facilitar, diremos apenas que o domínio $\Omega$ é $C^{m}$-regular em $\mathbb{R}^{N}$.

Recordando que a restrição de funções em $C_{o}^{\infty}\left(\mathbb{R}^{N}\right)$ a $\Omega$ é densa em $W^{m, p}(\Omega)$, para $u \in$ $C_{o}^{\infty}\left(\mathbb{R}^{N}\right)$ definimos a seguinte aplicação linear

$$
u \mapsto \gamma u=\left(\gamma_{0} u, \gamma_{1} u, \cdots, \gamma_{m-1} u\right), \quad \gamma_{j} u=\left.\frac{\partial^{j} u}{\partial n^{j}}\right|_{\partial \Omega},
$$

onde $\left.\frac{\partial^{j} u}{\partial n^{j}}\right|_{\partial \Omega}$ denota a restrição a $\partial \Omega$ da $j$-ésima derivada direcional na direção da normal interior a $\partial \Omega$.

Teorema 1.35. Se $\Omega$ é um domínio $C^{m}$-regular em $\mathbb{R}^{N}$ e $1<p<\infty$, então a aplicação $\gamma$ dada em 1.13 pode ser estendida por continuidade a um isomorfismo e um homeomorfismo de $W^{m, p}(\Omega) /$ Ker $\gamma$ sobre $\prod_{k=0}^{m-1} W^{m-k-\frac{1}{p}, p}(\partial \Omega)$.

Ao observarmos o Teorema 1.35 podemos considerar a aplicação

$$
W^{m, p} \ni u \stackrel{i}{\mapsto} i(u)=\left.u\right|_{\partial \Omega} \in W^{m-\frac{1}{p}, p}(\partial \Omega)
$$

e assim constatarmos que ele nos fornece ambas implicações, direta e recíproca, de teoremas de imersão para o espaço $W^{m, p}(\Omega)$ no sentido de que a aplicação $i$ mapeia continuamente o espaço $W^{m, p}(\Omega)$ no espaço $W^{m-\frac{1}{p}, p}(\partial \Omega)$, e reciprocamente, se $v$ é uma função em $W^{m-\frac{1}{p}}(\partial \Omega)$, então existe uma função $u \in W^{m, p}(\Omega) \operatorname{com} v=\left.u\right|_{\partial \Omega}$ e ainda

$$
\|u\|_{W^{m, p}(\Omega)} \leqslant K\|v\|_{W^{m-\frac{1}{p}}}
$$

ou seja, o espaço $W^{m, p}(\Omega)$ também é mapeado continuamente no espaço $W^{m-\frac{1}{p}}(\partial \Omega)$.

Uma abordagem mais detalhada do Teorema do Traço e suas implicações pode ser encontrada em Adams [1]. 


\section{Capítulo}

\section{Equações de reação e difusão com condições de fronteira não-lineares}

Nosso objetivo neste capítulo é escrever o problema proposto numa forma semilinear abstrata e introduzir os espaços de funções que nos serão adequados para estudá-lo. Com este intuito, reservamos a primeira seção para combinar resultados de existência, unicidade e regularidade de soluções para equações de reação e difusão com condições de fronteira não-lineares e também estudamos existência e limitação uniforme de atratores para semigrupos gerados por estas equações. Nas Seções 2.2 e 2.3 empregamos os resultados obtidos na seção anterior aos problemas $(2.6)$ e (2.8).

\subsection{Problemas parabólicos com condições de fronteira não- lineares}

Sejam $\Omega$ um domínio limitado e suave do $\mathbb{R}^{n}, \Gamma=\partial \Omega$ e consideremos o seguinte problema de reação e difusão com condições não-lineares na fronteira

$$
\begin{cases}u_{t}-\operatorname{div}(p(x) \nabla u)+\lambda u=f(u) & \text { em } \Omega \\ p(x) \frac{\partial u}{\partial \vec{n}}=g(u) & \text { em } \Gamma\end{cases}
$$

onde $p \in \mathscr{C}^{1}(\bar{\Omega})$ e as não-linearidades $f, g: \mathbb{R} \rightarrow \mathbb{R}$ satisfazem determinadas condições de crescimento e dissipatividade que caracterizaremos posteriormente.

Para estudar o comportamento assintótico das soluções da equação (2.1), precisamos determinar em que espaços trabalharemos. Com este objetivo, introduzimos nesta seção espaços de funções e operadores para que o problema (2.1) possa ser escrito em uma forma semilinear abstrata. 
Consideraremos os espaços Sobolev usuais $H^{1}(\Omega)$ e $W^{1, p}(\Omega)$, e os espaços de traço $H^{\frac{1}{2}}(\Gamma)$ e $W^{1-\frac{1}{p}, p}(\Gamma)$. Denotaremos por $H^{-s}$ o espaço dual de $H^{s}$, para $\Omega$ ou $\Gamma$. O produto de dualidade entre estes espaços será denotado por $\langle,\rangle_{-s, s}$ e, em particular, $\langle$,$\rangle denotará o produto em$ $L^{2}$, sendo que escreveremos $\langle,\rangle_{\Omega} \mathrm{e}\langle,\rangle_{\Gamma}$ para diferenciar se nos referimos a funções sobre $\Omega$ ou $\Gamma$ quando se fizer necessário.

Denotaremos por $\gamma$ o operador traço definido em $H^{s}(\Omega)$, com valores em $H^{s-\frac{1}{2}}(\Gamma)$, para $s>\frac{1}{2}$. Além disso, para uma função $u \in H^{s}(\Omega)$, identificaremos seu traço, $\gamma(u) \in H^{s-\frac{1}{2}}(\Gamma)$, com a forma linear $\gamma(u) \in H^{s-\frac{1}{2}}(\Gamma) \subset H^{-s}(\Omega)$ de tal forma que, para qualquer $\phi \in H^{s}(\Omega)$,

$$
\langle\gamma(u), \phi\rangle_{-s, s}:=\langle u, \phi\rangle_{\Gamma}:=\int_{\Gamma} \gamma(u) \gamma(\phi) d x
$$

ou seja, usamos as imersões $H^{s-\frac{1}{2}}(\Gamma) \subset L^{2}(\Gamma) \subset H^{-s}(\Omega) \subset H^{-1}(\Omega)$. Além disso, do Teorema do Traço, o operador traço $\gamma: W^{1, q}(\Omega) \rightarrow W^{1-\frac{1}{q}}(\Gamma)$ é um isomorfismo.

Consideraremos também o operador derivada normal, relativo ao operador difusão $-\operatorname{div}(p(x)$ $\nabla u)$, definido da seguinte forma: se

$$
u \in Z:=\left\{z \in H^{1}(\Omega):-\operatorname{div}(p(x) \nabla z) \in L^{2}(\Omega)\right\}
$$

então $\frac{\partial u}{\partial \vec{n}} \in H^{-\frac{1}{2}}(\Gamma)$ e é definido por

$$
\begin{aligned}
\left\langle\frac{\partial u}{\partial \vec{n}}, \gamma(v)\right\rangle_{-\frac{1}{2}, \frac{1}{2}} & =-\int_{\Omega}-\operatorname{div}(p(x) \nabla u) v d x+\int_{\Omega} p(x) \nabla u \nabla v d x \\
& =\int_{\Omega} \operatorname{div}(p(x) v \nabla u) d x
\end{aligned}
$$

qualquer que seja $v \in H^{1}(\Omega)$.

Sob estas condições e assumindo que $\lambda>0$, introduzimos o isomorfismo isométrico canônico entre $H^{1}(\Omega)$ e seu dual, $H^{-1}(\Omega)$, de tal maneira que, para $u, \phi \in H^{1}(\Omega)$,

$$
\langle L(u), \phi\rangle_{-1,1}=\int_{\Omega} p(x) \nabla u \nabla \phi d x+\int_{\Omega} \lambda u \phi d x .
$$

Desta forma, podemos reescrever (2.2) como

$$
\langle L(u), v\rangle_{-1,1}=\langle-\operatorname{div}(p(x) \nabla u)+\lambda u, v\rangle+\left\langle\frac{\partial u}{\partial \vec{n}}, \gamma(v)\right\rangle_{-\frac{1}{2}, \frac{1}{2}} .
$$

Também consideramos em $H^{1}(\Omega)$ o produto escalar

$$
a(u, v)=\int_{\Omega} p(x) \nabla u \nabla v d x=\langle L u, v\rangle_{-1,1}
$$


que nos fornece uma norma em $H^{1}(\Omega)$, equivalente à norma usual.

Suponhamos que $u$ seja uma solução de (2.1) e seja $\varphi \in H^{1}(\Omega)$. Então

$$
u_{t} \varphi-\operatorname{div}(p(x) \nabla u) \varphi+\lambda u \varphi=f(u) \varphi
$$

de modo que integrando por partes resulta que

$$
\int_{\Omega} u_{t} \varphi d x+\int_{\Omega} p(x) \nabla u \nabla \varphi d x+\int_{\Omega} \lambda u \varphi d x=\int_{\Omega} f(u) \varphi d x+\int_{\Gamma} g(\gamma(u)) \gamma(\varphi) d x .
$$

Notemos também que

$$
\langle g(\gamma(u), \gamma(\varphi))\rangle_{\Gamma}=\int_{\Gamma} g(\gamma(u)) \gamma(\varphi) d x=\int_{\Gamma} \gamma(g(u) \gamma(\varphi)) d x=\langle\gamma(g(u)), \gamma(\varphi)\rangle_{-1,1} .
$$

Logo,

$$
\left\langle u_{t}, \varphi\right\rangle_{-1,1}+\langle A u, \varphi\rangle_{-1,1}=\langle f(u), \varphi\rangle_{-1,1}+\langle g(\gamma(u)), \gamma(\varphi)\rangle_{-1,1}
$$

onde $\langle,\rangle_{-1,1}$ representa o produto de dualidade entre $\left(H^{1}(\Omega)\right)^{\prime}$ e $H^{1}(\Omega)$ e $A u: H^{1}(\Omega) \rightarrow \mathbb{R}$, $\operatorname{com} H^{1}(\Omega) \ni \varphi \mapsto\langle A u, \varphi\rangle=\int_{\Omega} p(x) \nabla u \nabla \varphi d x+\int_{\Omega} \lambda u \varphi d x$

Em $L^{2}(\Omega)$, definimos $A: \mathscr{D}(A) \subset L^{2}(\Omega) \rightarrow L^{2}(\Omega)$ como o operador dado por

$$
\begin{aligned}
\mathscr{D}(A) & =\left\{u \in H^{2}(\Omega): \frac{\partial u}{\partial \vec{n}}=0 \text { em } \Gamma\right\} \\
A u & =-\operatorname{div}(p(x) \nabla u)+\lambda u, \quad u \in \mathscr{D}(A),
\end{aligned}
$$

$\operatorname{com} \lambda>0$.

Assim, podemos escrever o problema (2.1) na forma abstrata

$$
\left\{\begin{array}{l}
u_{t}+A u=h(u) \\
u(0)=u_{0}
\end{array}\right.
$$

onde $h$ é definida como $h(u)=f_{\Omega}(u)+g_{\Gamma}(u)$, no sentido que

$$
\left\langle f_{\Omega}(u)+g_{\Gamma}(u), \phi\right\rangle=\int_{\Omega} f(u) \phi d x+\int_{\Gamma} g(\gamma(u)) \gamma(\phi) d x,
$$

para funções-teste $\phi$ escolhidas apropriadamente.

Proposição 2.1. O operador A definido acima é positivo, auto-adjunto, e tem resolvente compacto em $L^{2}(\Omega)$. Em particular, A é um operador setorial em $L^{2}(\Omega)$ e suas potências fracionárias verificam $X^{\alpha} \subset H^{2 \alpha}(\Omega)$, para $\alpha \geqslant 0$ e em particular

$$
X^{1}=\mathscr{D}(A), \quad H^{\frac{1}{2}}=H^{1}(\Omega), \quad X^{0}=L^{2}(\Omega), \quad X^{-\frac{1}{2}}=H^{-1}(\Omega),
$$


onde convencionamos $H^{-1}(\Omega):=\left(H^{1}(\Omega)\right)^{\prime}$.

A realização de $L$ em $L^{2}(\Omega)$, ou seja, a restrição de $L$ ao domínio $\mathscr{D}=\left\{u \in H^{1}(\Omega): L u \in\right.$ $\left.L^{2}(\Omega)\right\}$ coincide com A e $\mathscr{D}=\mathscr{D}(A)$. Além disso, L é um operador setorial em $H^{-1}(\Omega)$ com domínio $H^{1}(\Omega)$.

\subsubsection{Existências local e global de solução}

Os resultados e a notação que serão utilizados nesta subseção são provenientes de Carvalho, Oliva, Pereira e Rodríguez-Bernal [10]. Para mais detalhes podem ser consultados também Arrieta, Carvalho e Rodríguez-Bernal [4, 5].

Suponhamos que $R$ seja um operador setorial em um espaço de Hilbert $X, \operatorname{com} \operatorname{Re}(\sigma(\mathrm{R}))>$ 0 . Podemos então definir as potências fracionárias $R^{\alpha}$ de $R$, bem como os espaços de potência fracionária $X^{\alpha}:=\mathscr{D}\left(R^{\alpha}\right)$, munidos da norma do gráfico, $\alpha \in \mathbb{R}$, onde $X^{-\alpha}=\left(X^{\alpha}\right)^{\prime}$, para $\alpha>0$. Além disso, $R$ é setorial em $X^{\alpha}$ com domínio $X^{\alpha+1}$, para qualquer $\alpha$, veja Henry [15].

Teorema 2.2. Suponhamos que $h: X^{\alpha} \rightarrow X^{\beta}$ seja localmente Lipschitz e limitada sobre conjuntos limitados, onde $0 \leqslant \alpha-\beta<1$. então o problema parabólico abstrato

$$
\left\{\begin{array}{l}
u_{t}+R u=h(u) \\
u(0)=u_{0} \in X^{\alpha}
\end{array}\right.
$$

tem uma única solução localmente definida, dada pela Fórmula da Variação das Constantes

$$
u(t)=e^{-R t} u_{0}+\int_{0}^{t} e^{-R(t-r)} h(u(r)) d r
$$

onde $e^{-R t}$ denota o semigrupo analítico gerado por $-R$. Além disso, $u$ verifica

$$
u \in C\left([0, T), X^{\alpha}\right) \cap C\left((0, T), X^{\beta+1}\right), \quad u_{t} \in C\left((0, T), X^{\gamma}\right)
$$

para todo $\gamma<\beta+1$ e a equação é verificada em $X^{\beta}$. Além disso, ou a solução está definida para todo $t \geqslant 0$, ou a solução "explode", na norma $X^{\alpha}$, em tempo finito.

Aplicamos este resultado ao operador $A$ definido na seção anterior, considerando, para tanto, aplicações não-lineares da forma $h(u):=f_{\Omega}(u)+g_{\Gamma}(u)$, onde, ao menos, $f_{\Omega}: X^{\alpha} \rightarrow L^{2}(\Omega)$ e $g_{\Gamma}: X^{\alpha} \rightarrow H^{-\frac{1}{2}}(\Gamma)$, para algum $\alpha>0$. Notemos que $h$ age em funções teste $\phi \in H^{r}(\Omega)$, para $r>\frac{1}{2}$, como $\langle h(u), \phi\rangle=\left\langle f_{\Omega}(u), \phi\right\rangle_{\Omega}+\left\langle g_{\Gamma}(u), \phi\right\rangle_{\Gamma}$. Dependendo das propriedades extras de regularidade de $g_{\Gamma}$, iremos considerar

$$
h: X^{\alpha} \rightarrow X^{\beta}
$$


para $\alpha>0$ e $\beta \leqslant 0$ escolhidos apropriadamente. Investiguemos tais valores apropriados, lembrando primeiramente que, para os resultados abstratos, devemos ter $0 \leqslant \alpha-\beta<1$.

Como estamos considerando o caso em que $g \neq 0$, devemos ter $\beta<0$, caso contrário, poderíamos tomar $\beta=0$. Para que possamos ler a equação em $H^{-1}(\Omega)$, vamos considerar também $-\frac{1}{2} \leqslant \beta<0$. As estimativas de energia na solução exigem que $u, u_{t} \in H^{1}(\Omega)$, para $t>0$, e então segue do Teorema 2.2 que $\beta$ deve satisfazer $\beta+1>\frac{1}{2}$, ou seja, $\beta>-\frac{1}{2}$.

Por outro lado, se queremos que o termo não-linear $g(u)$ dependa dos valores de $u$ em $\Gamma$, devemos ter $\alpha>\frac{1}{4}$ a fim de que o traço de $u$ esteja bem definido; caso contrário, podemos tomar $\alpha \geqslant 0$. Pelo fato de querermos que o dado inicial esteja ao menos em $H^{1}(\Omega)$, precisamos ter $\alpha \geqslant \frac{1}{2}$.

Notemos, finalmente, que no caso de termos não-nulos na fronteira, existem, naturalmente, limitações superiores para $\alpha$ e $\beta$. Em verdade, $\alpha<\frac{3}{4}$, já que para $\alpha \geqslant \frac{3}{4}$, o espaço $X^{\alpha}$ incorpora a condição de fronteira $\frac{\partial u}{\partial \vec{n}}=0$ e, com relação à $\beta$, da regularidade no Teorema 2.2, devemos ter $\beta+1<\frac{3}{4}$, ou seja, $\beta<-\frac{1}{4}$.

Em resumo, se $g \neq 0$, então

$$
\frac{1}{4}<\alpha<\frac{3}{4}, \quad-\frac{1}{2}<\beta<-\frac{1}{4} \quad \text { e } \quad 0 \leqslant \alpha-\beta<1,
$$

enquanto que, se $g=0$, temos o caso canônico $\beta=0,0 \leqslant \alpha<1$.

Com isto, temos o seguinte resultado:

Teorema 2.3. Suponhamos que $f_{\Omega}, g_{\Gamma}, \alpha$ e $\beta$ satisfaçam as condições acima e

$$
h: X^{\alpha} \rightarrow X^{\beta}
$$

seja localmente Lipschitz contínua e limitada sobre conjuntos limitados. Então, para cada $u_{0} \in X^{\alpha}$, existe uma única solução localmente definida de

$$
\left\{\begin{array}{l}
u_{t}+A u=h(u) \\
u(0)=u_{0} \in X^{\alpha}
\end{array}\right.
$$

dada por

$$
u(t)=e^{-A t} u_{0}+\int_{0}^{t} e^{-A(t-r)} h(u(r)) d r
$$

onde $e^{-A t}$ denota o semigrupo analítico gerado por - A que verifica

$$
u \in C\left([0, T), X^{\alpha}\right) \cap C\left((0, T), X^{\beta+1}\right), \quad u_{t} \in C\left((0, T), X^{\gamma}\right)
$$


para todo $\gamma<\beta+1 e$

$$
\int_{\Omega} u_{t} \phi d x+\int_{\Omega} p(x) \nabla u \nabla \phi d x+\int_{\Omega} \lambda u \phi d x+\int_{\Omega} f(u) \phi d x+\langle g(\gamma(u)), \gamma(\phi)\rangle_{\Gamma}
$$

para toda $\phi \in H^{1}(\Omega)$. Em particular, temos

$$
\begin{cases}u+A u=f(u) & \text { em } \Omega \\ p(x) \frac{\partial u}{\partial \vec{n}}=g(u) & \text { em } \Gamma\end{cases}
$$

e, ou a solução está definida para todo $t>0$, ou ela explode em tempo finito na norma $X^{\alpha}$.

Em particular, se

$$
\begin{aligned}
& f_{\Omega}: X^{\alpha} \rightarrow L^{2}(\Omega) \\
& g_{\Gamma}: X^{\alpha} \rightarrow L^{2}(\Gamma) \quad \text { ou } \quad H^{-r}(\Gamma), \quad \text { com } \quad 0 \leqslant r \leqslant \frac{1}{2}
\end{aligned}
$$

são funções não-lineares localmente Lipschitz, para $\alpha+\frac{r}{2}<\frac{3}{4}$, então existe $\beta$ tal que $-\frac{1}{2}<$ $\beta<-\frac{1}{4}$ verifica todas as afirmações acima.

Consideremos agora a condição de crescimento :

(C) Se $n=2$, assumimos que para cada $\eta>0$ existe uma constante $C_{\eta}>0$ tal que, para $j=f, g$, temos

$$
|j(u)-j(v)| \leqslant C_{\eta}\left(e^{\eta|u|^{2}}+e^{\eta|v|^{2}}\right)|u-v|, \text { para quaisquer } u, v \in \mathbb{R}
$$

ou, se $n \geqslant 3$, assumimos que existe uma constante $C>0$ tal que

$$
\begin{aligned}
& |f(u)-f(v)| \leqslant C|u-v|\left(|u|^{\frac{4}{n-2}}+|v|^{\frac{4}{n-2}}+1\right) \\
& |g(u)-g(v)| \leqslant C|u-v|\left(|u|^{\frac{2}{n-2}}+|v|^{\frac{2}{n-2}}+1\right),
\end{aligned}
$$

para quaisquer $u, v \in \mathbb{R}$.

Sob estas condições, o problema (2.1) está localmente bem posto em $X^{\alpha}$.

Para obtermos que as soluções de (2.1) estão globalmente definidas, assumiremos a seguinte condição de sinal sobre os termos não-lineares:

(S) Assumamos que existam $B_{0}, C_{0} \in \mathbb{R}$ e $B_{1}, C_{1} \leqslant 0$ de tal forma que

$$
u f(u) \leqslant-C_{0} u^{2}+C_{1}|u|
$$




$$
u g(u) \leqslant-B_{0} u^{2}+B_{1}|u|
$$

para todo $u \in \mathbb{R}$.

Então temos o seguinte resultado acerca da existência global das soluções:

Teorema 2.4. Assumamos que as condições de crescimento $(\mathbf{C})$ e de sinal $\mathbf{( S )}$ estejam satisfeitas. Então, para todo $u_{0} \in X^{\alpha}$ a solução $u\left(t, u_{0}\right)$ da equação (2.1) começando em $u_{0}$ existe para $t \geqslant 0$.

\subsubsection{Existência de atratores e estimativas uniformes}

Para provarmos a existência de um atrator global para o problema (2.1) devemos assumir também uma condição de dissipatividade, que apresentamos a seguir. Em Arrieta, Carvalho e Rodríguez-Bernal [3, 4, 5] podem ser encontrados mais detalhes sobre existência e limitação uniforme de atratores para este problema.

(D) Assumamos que a condição (S) esteja satisfeita e sejam $C_{0}$ e $B_{0}$ as constantes de (S). Assumamos que seja positivo o primeiro autovalor, $\mu_{1}$, do problema

$$
\begin{cases}-\operatorname{div}(p(x) \nabla u)+\left(\lambda+C_{0}\right) u=\mu u & \text { em } \Omega \\ p(x) \frac{\partial u}{\partial \vec{n}}+B_{0} u=0 & \text { em } \Gamma .\end{cases}
$$

Sob esta condição, temos o seguinte resultado:

Teorema 2.5. Suponhamos que as condições de crescimento $(\mathbf{C})$, de sinal $\mathbf{( S )}$ e de dissipatividade (D) sejam válidas. Então o semigrupo $\{T(t): t \geqslant 0\}$ associado a (2.1) tem um atrator global, $\mathscr{A}$, em $X^{\alpha}$.

Além disso, este atrator é uniformemente limitado em $L^{\infty}(\Omega)$. Mais precisamente, temos o seguinte resultado:

Proposição 2.6. Suponhamos que as condições de crescimento (C) e dissipatividade (D) estejam satisfeitas. Denotemos por $\phi$ a solução de

$$
\begin{cases}\operatorname{div}(p(x) \nabla \phi)+\left(\lambda+C_{0}\right) \phi=C_{1}, & \text { em } \Omega \\ p(x) \frac{\partial \phi}{\partial \vec{n}}+B_{0} \phi=B_{1} & \text { em } \Gamma .\end{cases}
$$

Então $0 \leqslant \phi \in L^{\infty}(\Omega), \lim _{t \rightarrow \infty}\left|u\left(t, x, u_{0}\right)\right| \leqslant \phi(x)$, uniformemente em $x \in \bar{\Omega}$ e para $u_{0}$ em subconjuntos limitados de $X^{\alpha}$. Em particular, para cada $v \in \mathscr{A}$, temos $|v(x)| \leqslant \phi(x)$. 


\subsection{Problema perturbado}

Sejam $\Omega \subset \mathbb{R}^{n}$ um domínio limitado e suave, $\Gamma=\partial \Omega$ e $\varepsilon \in\left(0, \varepsilon_{0}\right]$ um parâmetro. Consideremos a equação de reação e difusão

$$
\begin{cases}u_{t}^{\varepsilon}-\operatorname{div}\left(p_{\varepsilon}(x) \nabla u^{\varepsilon}\right)+\lambda u^{\varepsilon}=f\left(u^{\varepsilon}\right) & \text { em } \Omega \\ p_{\varepsilon}(x) \frac{\partial u^{\varepsilon}}{\partial \vec{n}}=g\left(u^{\varepsilon}\right) & \text { em } \Gamma \\ u^{\varepsilon}(0)=u_{0}^{\varepsilon} & \end{cases}
$$

onde $\lambda>0$, as não-linearidades $f, g \in \mathscr{C}^{2}(\mathbb{R})$ satisfazem as condições e crescimento (C) e de sinal (S) dadas na seção anterior e os coeficientes de difusão $p_{\varepsilon}: \Omega \subset \mathbb{R}^{n} \rightarrow \mathbb{R}$ são funções regulares e limitadas em $\Omega$, satisfazendo

$$
0<m_{0} \leqslant p_{\varepsilon}(x) \leqslant M_{\varepsilon}
$$

para todo $x \in \Omega$ e $0<\varepsilon \leqslant \varepsilon_{0}$.

Procedendo de modo inteiramente análogo à seção anterior, podemos reescrever o problema (2.6) como uma equação semilinear abstrata

$$
\left\{\begin{array}{l}
u_{t}^{\varepsilon}+A_{\varepsilon} u^{\varepsilon}=h\left(u^{\varepsilon}\right) \\
u^{\varepsilon}(0)=u_{0}^{\varepsilon}
\end{array}\right.
$$

onde, para $X=L^{2}(\Omega), A_{\varepsilon}$ é o operador linear dado por

$$
\begin{aligned}
A_{\varepsilon}: \mathscr{D}\left(A_{\mathcal{\varepsilon}}\right) \subset X & \rightarrow X \\
u & \mapsto A_{\varepsilon} u=-\operatorname{div}\left(p_{\varepsilon}(x) \nabla u\right)+\lambda u,
\end{aligned}
$$

sendo $\mathscr{D}\left(A_{\varepsilon}\right)=\left\{u \in \mathrm{H}^{1}(\Omega):-\operatorname{div}\left(p_{\varepsilon}(x) \nabla u\right) \in X, \frac{\partial u}{\partial \vec{n}}=0\right.$ em $\left.\Gamma\right\}$ e $h$ definida como $h(u)=$ $f_{\Omega}(u)+g_{\Gamma}(u)$, no sentido que

$$
\left\langle f_{\Omega}(u)+g_{\Gamma}(u), \phi\right\rangle=\int_{\Omega} f(u) \phi d x+\int_{\Gamma} g(\gamma(u)) \gamma(\phi) d x,
$$

para funções-teste $\phi$ escolhidas apropriadamente.

Vejamos algumas propriedades dos operadores $A_{\varepsilon}, \varepsilon \in\left(0, \varepsilon_{0}\right]$. O resultado seguinte pode ser encontrado em Rodríguez-Bernal [18]:

Teorema 2.7. Para $\lambda>0$, o operador $B_{\mathcal{\varepsilon}}$, definido pela forma bilinear

$$
a_{\varepsilon}(u, v)=\left\langle B_{\varepsilon} u, v\right\rangle_{-1,1}=\int_{\Omega} p_{\varepsilon}(x) \nabla u \nabla v d x+\int_{\Omega} \lambda u v d x
$$


é um isomorfismo entre $H^{1}(\Omega)$ e seu dual $H^{-1}(\Omega)$. A realização de $B_{\varepsilon}$ em $L^{2}(\Omega)$ sob condição de Neumann na fronteira, que denotaremos por $A_{\mathcal{E}}$, é um operador positivo, auto-adjunto e com resolvente compacto, com domínio

$$
\mathscr{D}\left(A_{\varepsilon}\right)=\left\{u \in \mathrm{H}^{1}(\Omega):-\operatorname{div}\left(p_{\varepsilon}(x) \nabla u\right) \in \mathrm{L}^{2}(\Omega), \frac{\partial u}{\partial \vec{n}}=0 \text { em } \Gamma\right\},
$$

e para $u \in \mathscr{D}\left(A_{\varepsilon}\right)$,

$$
A_{\varepsilon} u=-\operatorname{div}\left(p_{\varepsilon}(x) \nabla u\right)+\lambda u
$$

Demonstração. Observemos inicialmente que a forma bilinear $a_{\varepsilon}$ é coerciva em $H^{1}(\Omega)$ para $\lambda>0$, pois

$$
\begin{aligned}
a_{\varepsilon}(u, u) & =\int_{\Omega} p_{\varepsilon}(x)|\nabla u|^{2} d x+\int_{\Omega} \lambda|u|^{2} d x \\
& \geqslant m_{0} \int_{\Omega}|\nabla u|^{2} d x+\int_{\Omega} \lambda|u|^{2} d x \\
& \geqslant \min \left\{m_{0}, \lambda\right\}\left(\int_{\Omega}|\nabla u|^{2} d x+\int_{\Omega}|u|^{2} d x\right) \\
& =C\|u\|_{H^{1}(\Omega)}^{2} .
\end{aligned}
$$

Desta forma, $B_{\varepsilon}$ é um operador injetivo, já que $\left\langle B_{\varepsilon} u, u\right\rangle=0$ implica $u=0$. Claramente, a forma bilinear $a_{\varepsilon}$ é simétrica, e a continuidade segue de

$$
\begin{aligned}
a_{\mathcal{\varepsilon}}(u, v) & =\int_{\Omega} p_{\mathcal{\varepsilon}}(x) \nabla u \nabla v d x+\int_{\Omega} \lambda u v d x \\
& \leqslant M_{\mathcal{E}}\|\nabla u\|_{L^{2}(\Omega)}\|\nabla v\|_{L^{2}(\Omega)}+\lambda\|u\|_{L^{2}(\Omega)}\|v\|_{L^{2}(\Omega)} \\
& \leqslant \max \left\{M_{\mathcal{E}}, \lambda\right\}\|u\|_{H^{1}(\Omega)}\|v\|_{H^{1}(\Omega)} .
\end{aligned}
$$

Segue então que $B_{\varepsilon} \in \mathscr{L}\left(H^{1}(\Omega), H^{-1}(\Omega)\right)$ e, pelo Teorema de Lax-Milgram, qualquer que seja $\varphi \in H^{-1}(\Omega)$, existe um único $u \in H^{1}(\Omega)$ tal que

$$
a_{\varepsilon}(u, v)=\langle\varphi, v\rangle, \quad \text { para todo } v \in H^{1}(\Omega),
$$

ou seja,

$$
\left\langle B_{\varepsilon} u, v\right\rangle=\langle\varphi, v\rangle, \quad \text { para todo } v \in H^{1}(\Omega)
$$

e $B_{\varepsilon} u=\varphi$ em $H^{1}(\Omega)$, o que mostra que o operador $B_{\varepsilon}$ é um isomorfismo. 
Consideremos agora o operador $A_{\varepsilon}: \mathscr{D}\left(A_{\varepsilon}\right) \subset L^{2}(\Omega) \rightarrow L^{2}(\Omega)$. O domínio de $A_{\varepsilon}$ é dado de forma natural por

$$
\mathscr{D}\left(A_{\varepsilon}\right)=\left\{u \in H^{1}(\Omega): B_{\varepsilon} u \in L^{2}(\Omega), \frac{\partial u}{\partial \vec{n}}=0 \mathrm{em} \Gamma\right\} .
$$

Vamos mostrar que $\mathscr{D}\left(A_{\mathcal{\varepsilon}}\right)=H_{0}^{1}(\Omega) \cap\left\{u \in H^{2}(\Omega): \frac{\partial u}{\partial \vec{n}}=0\right.$ em $\left.\Gamma\right\}$. Para tanto, seja $u \in$ $\mathscr{D}\left(A_{\mathcal{\varepsilon}}\right)$. Então $B_{\mathcal{\varepsilon}} u \in L^{2}(\Omega)$ e, para toda $\varphi \in H^{1}(\Omega)$, temos

$$
\int_{\Omega} p_{\varepsilon}(x) \nabla u^{\varepsilon} \nabla \varphi d x+\int_{\Omega} \lambda u \varphi d x=\int_{\Omega} B_{\varepsilon} u \varphi d x,
$$

ou seja, $u$ é solução fraca do problema de Neumann

$$
\begin{cases}-\operatorname{div}\left(p_{\varepsilon}(x) \nabla u\right)+\lambda u=B_{\varepsilon} u, & \text { em } \Omega \\ p_{\varepsilon}(x) \frac{\partial u}{\partial \vec{n}}=0 & \text { em } \Gamma\end{cases}
$$

e $u \in H^{2}(\Omega)$. A outra inclusão é imediata. Podemos então escrever

$$
\begin{aligned}
\left\langle B_{\varepsilon} u, v\right\rangle_{L^{2}(\Omega)} & =\int_{\Omega} p_{\varepsilon}(x) \nabla u \nabla v d x+\int_{\Omega} \lambda u v d x \\
& =\int_{\Omega}-\operatorname{div}\left(p_{\varepsilon}(x) \nabla u\right) v d x+\int_{\Gamma} p_{\varepsilon}(x) \frac{\partial u}{\partial \vec{n}} v d x+\int_{\Omega} \lambda u v d x \\
& =\left\langle-\operatorname{div}\left(p_{\varepsilon}(x) \nabla u\right)+\lambda u, v\right\rangle_{L^{2}(\Omega)}
\end{aligned}
$$

Como $u \in L^{2}(\Omega)$, dizer que $B_{\varepsilon} u \in L^{2}(\Omega)$ equivale a dizer que $-\operatorname{div}\left(p_{\varepsilon}(x) \nabla u\right) \in L^{2}(\Omega)$. $\operatorname{Logo}, \mathscr{D}\left(A_{\varepsilon}\right)=\left\{u \in H^{1}(\Omega):-\operatorname{div}\left(p_{\varepsilon}(x) \nabla u\right) \in L^{2}(\Omega), \frac{\partial u}{\partial \vec{n}}=0\right.$ em $\left.\Gamma\right\}$ e $A_{\varepsilon}$ é dado por

$$
A_{\varepsilon} u=-\operatorname{div}\left(p_{\varepsilon}(x) \nabla u\right)+\lambda u, \text { para todo } u \in \mathscr{D}\left(A_{\varepsilon}\right) .
$$

Ainda, a identidade $a_{\varepsilon}(u, v)=\left\langle A_{\varepsilon} u, v\right\rangle_{L^{2}(\Omega)}$ nos dá que o operador $A_{\varepsilon}$ é positivo e simétrico. Como $A_{\varepsilon}$ é sobrejetivo, segue que $A_{\varepsilon}$ é auto-adjunto.

Resta-nos verificar que $A_{\varepsilon}$ tem resolvente compacto. Como $A_{\varepsilon}$ é bijetivo e fechado, temos que $A_{\varepsilon}{ }^{-1}$ é fechado, e portanto segue do Teorema do Gráfico Fechado que $A_{\varepsilon}{ }^{-1}$ é contínuo. Logo, $0 \in \rho\left(A_{\varepsilon}\right)$, e então basta provarmos que $A_{\varepsilon}{ }^{-1}$ é compacto. Para tanto, seja $\left\{g_{\varepsilon}\right\} \subset L^{2}(\Omega)$ tal que $\left\|g_{\varepsilon}\right\|_{L^{2}(\Omega)} \leqslant 1$. Temos

$$
\left\langle A_{\varepsilon} v^{\varepsilon}, v^{\varepsilon}\right\rangle=\int_{\Omega} p_{\varepsilon}(x)\left|\nabla v^{\varepsilon}\right|^{2} d x+\int_{\Omega} \lambda\left|v^{\varepsilon}\right|^{2} d x=\int_{\Omega} g_{\varepsilon} v^{\varepsilon} d x,
$$


e assim, se $C_{1}=\min \left\{m_{0}, \lambda\right\}$ e $C_{2}$ é a constante de imersão de $H^{1}(\Omega)$ em $L^{2}(\Omega)$, então segue da Desigualdade de Young que

$$
C_{1}\left\|v^{\varepsilon}\right\|_{H^{1}(\Omega)}^{2} \leqslant \int_{\Omega} p_{\varepsilon}(x)\left|\nabla v^{\varepsilon}\right|^{2} d x+\lambda\left\|v^{\varepsilon}\right\|_{L^{2}(\Omega)}^{2} \leqslant \frac{2}{\lambda}\left\|g_{\varepsilon}\right\|_{L^{2}(\Omega)}^{2}+\frac{C_{2} \lambda}{2}\left\|v^{\varepsilon}\right\|_{L^{2}(\Omega)}^{2}
$$

e

$$
\left\|v^{\varepsilon}\right\|_{H^{1}(\Omega)}^{2} \leqslant C_{3}
$$

onde $C_{3}=\frac{2}{\lambda}\left(C_{1}-\frac{C_{2} \lambda}{2}\right)^{-1}$.

Logo, $A_{\varepsilon}^{-1} \in \mathscr{L}\left(L^{2}(\Omega), H^{1}(\Omega)\right)$ e portanto é um operador compacto de $L^{2}(\Omega)$ nele mesmo, já que a inclusão $H^{1}(\Omega) \hookrightarrow L^{2}(\Omega)$ é compacta.

Deste modo, o operador $\left(\mu-A_{\mathcal{\varepsilon}}\right)^{-1}$ é compacto, para todo $\mu \in \rho\left(A_{\varepsilon}\right)$.

Teorema 2.8. Para cada $\varepsilon \in\left(0, \varepsilon_{0}\right]$, o operador $A_{\varepsilon}: \mathscr{D}\left(A_{\varepsilon}\right) \subset L^{2}(\Omega) \rightarrow L^{2}(\Omega)$ é setorial e

$$
\left\|\left(\mu+A_{\varepsilon}\right)^{-1}\right\|_{\mathscr{L}\left(L^{2}(\Omega)\right)} \leqslant \frac{M}{|\mu+\lambda|}, \quad \text { para todo } \mu \in \Sigma_{-\lambda, \phi},
$$

para alguma constante $M$ independente de $\varepsilon$.

Demonstração. Como $\left\langle A_{\varepsilon} u, u\right\rangle_{L^{2}(\Omega)} \geqslant \lambda\langle u, u\rangle_{L^{2}(\Omega)}$, resulta que o operador $-A_{\varepsilon}$ é dissipativo, satisfazendo $\left\langle-A_{\varepsilon} u, u\right\rangle_{L^{2}(\Omega)} \leqslant-\lambda\langle u, u\rangle_{L^{2}(\Omega)}$, para todo $u \in H^{1}(\Omega)$.

Temos que $W\left(-A_{\varepsilon}\right) \subset(-\infty,-\lambda]$, sendo

$$
W\left(-A_{\varepsilon}\right)=\left\{\left\langle-A_{\varepsilon} u, u\right\rangle_{L^{2}(\Omega)}: u \in \mathscr{D}\left(A_{\varepsilon}\right),\|u\|_{L^{2}(\Omega)}=1\right\}
$$

a imagem numérica de $-A_{\varepsilon}$. Desta forma, $\mathscr{R}:=\mathbb{C} \backslash(-\infty,-\lambda] \subset \mathbb{C} \backslash W\left(-A_{\varepsilon}\right)$ é aberto e conexo. Temos ainda que $0 \in \mathscr{R} \cap \rho\left(-A_{\mathcal{E}}\right)$. Do Teorema 1.7 resulta que, se $\mu \in \mathscr{R}$, então

$$
\left\|\left(\mu-\left(-A_{\varepsilon}\right)\right)^{-1}\right\|_{\mathscr{L}\left(L^{2}(\Omega)\right)} \leqslant \frac{1}{\operatorname{dist}\left(\mu, W\left(-A_{\varepsilon}\right)\right)} .
$$

Logo, se $0<\phi<\frac{\pi}{2}$ e $\mu \in \Sigma_{-\lambda, \phi}:=\{w \in \mathbb{C}:|\arg (w+\lambda)|<\pi-\phi\}$, temos

$$
\operatorname{dist}\left(\mu, W\left(-A_{\varepsilon}\right)\right) \geqslant \operatorname{dist}(\mu,(-\infty,-\lambda])=|\mu+\lambda| \sin (\pi-\arg (\mu+\lambda)) \geqslant|\mu+\lambda| \sin \phi,
$$

obtendo assim

$$
\left\|\left(\mu+A_{\varepsilon}\right)^{-1}\right\|_{\mathscr{L}\left(L^{2}(\Omega)\right)} \leqslant \frac{M}{|\mu+\lambda|}, \quad \text { para todo } \mu \in \Sigma_{-\lambda, \phi},
$$

em que $M=\frac{1}{\sin \phi}$ independe de $\varepsilon$. 


\subsection{Problema limite}

Sejam $\Omega_{0}$ um sub-domínio suave de $\Omega$, com $\bar{\Omega}_{0} \subset \Omega$ e $m$ um inteiro positivo tal que $\Omega_{0}=$ $\bigcup_{i=1}^{m} \Omega_{0, i}$, onde $\Omega_{0, i}$ são sub-domínios suaves e conexos de $\Omega$ com $\bar{\Omega}_{0, i} \cap \bar{\Omega}_{0, j}=\varnothing$, para $i \neq j$. Denotemos por $\Omega_{1}=\Omega \backslash \bar{\Omega}_{0}$, e $\Gamma_{0, i}=\partial \Omega_{0, i}, \Gamma_{0}=\cup_{i=1}^{m} \Gamma_{0, i}$ as fronteiras de $\Omega_{0, i}$ e $\Omega_{0}$, respectivamente. Notemos que $\partial \Omega_{1}=\Gamma \cup \Gamma_{0}$, onde $\Gamma=\partial \Omega$.

Assumimos que a difusão é grande em $\Omega_{0}$ quando $\varepsilon \rightarrow 0$, mais precisamente,

$$
p_{\varepsilon}(x) \rightarrow\left\{\begin{array}{cl}
p_{0}(x), & \text { uniformemente sobre } \Omega_{1},\left(p_{0} \in \mathscr{C}^{1}\left(\bar{\Omega}_{1},(0, \infty)\right)\right) ; \\
\infty, & \text { uniformemente sobre subconjuntos compactos de } \Omega_{0} .
\end{array}\right.
$$

Como vimos anteriormente, o problema limite de (2.6) quando $\varepsilon \rightarrow 0$ é dado por

$$
\begin{cases}u_{t}-\operatorname{div}\left(p_{0}(x) \nabla u\right)+\lambda u=f(u) & \text { em } \Omega_{1} \\ u_{\mid \Omega_{0, i}}:=u_{\Omega_{0, i}} & \text { em } \Omega_{0, i}, i=1, \ldots, m \\ \dot{u}_{\Omega_{0, i}}+\frac{1}{\left|\Omega_{0, i}\right|} \int_{\Gamma_{0, i}} p_{0}(x) \frac{\partial u}{\partial \vec{n}} d x+\int_{\Omega_{0, i}} \lambda u_{\Omega_{0, i}} d x=f\left(u_{\Omega_{0, i}}\right) & i=1, \ldots, m \\ p_{0}(x) \frac{\partial u}{\partial \vec{n}}=g(u) & \text { em } \Gamma \\ u(0)=u_{0} . & \end{cases}
$$

Seja $u$ uma solução de (2.8). Multiplicando a primeira equação por $\varphi \in H_{\Omega_{0}}^{1}(\Omega):=\{u \in$ $H^{1}(\Omega): u$ é constante em $\left.\Omega_{0}\right\}$ e usando que $\int_{\Omega} \operatorname{div}\left(p_{0}(x) \nabla u\right) \varphi d x=\int_{\Omega_{1}} \operatorname{div}\left(p_{0}(x) \nabla u\right) \varphi d x$, podemos escrever a equação (2.8) na forma semilinear abstrata

$$
\left\{\begin{array}{l}
u_{t}+A_{0} u=h(u) \\
u(0)=u_{0}
\end{array}\right.
$$

onde

$$
\begin{aligned}
A_{0} u & =\left(-\operatorname{div}\left(p_{0}(x) \nabla u\right)+\lambda u\right) \chi_{\Omega_{1}} \\
& +\sum_{i=1}^{m} \frac{1}{\left|\Omega_{0, i}\right|}\left(\int_{\Gamma_{0, i}} p_{0}(x) \frac{\partial u}{\partial \vec{n}} d x+\int_{\Omega_{0, i}} \lambda u_{\Omega_{0, i}}\right) \chi_{\Omega_{0, i}}
\end{aligned}
$$

com

$$
\mathscr{D}\left(A_{0}\right)=\left\{u \in \mathrm{H}_{\Omega_{0}}^{1}(\Omega):-\operatorname{div}\left(p_{0}(x) \nabla u\right) \in \mathrm{L}^{2}\left(\Omega_{1}\right), \frac{\partial u}{\partial \vec{n}}=0 \mathrm{em} \Gamma\right\} .
$$

Analogamente ao caso dos operadores $A_{\varepsilon}$ do problema perturbado, podemos demonstrar os seguintes resultados acerca do operador $A_{0}$ : 
Teorema 2.9. Para todo $\lambda>0$, a forma bilinear

$$
a_{0}(u, v)=\int_{\Omega_{1}} p_{0}(x) \nabla u \nabla v d x+\int_{\Omega} \lambda u v d x
$$

com $u, v \in H_{\Omega_{0}}^{1}(\Omega)$, define um isomorfismo $B_{0}$ entre $H_{\Omega_{0}}^{1}(\Omega)$ e seu dual $H_{\Omega_{0}}^{-1}(\Omega)$. A $L_{\Omega_{0}}^{2}(\Omega)$ realização de $B_{0}$ com condição de Neumann na fronteira é um operador não-limitado, positivo, auto-adjunto e com resolvente compacto $A_{0}$, com domínio

$$
\mathscr{D}\left(A_{0}\right)=\left\{u \in H_{\Omega_{0}}^{1}(\Omega):-\operatorname{div}\left(p_{0}(x) \nabla u\right) \in L^{2}\left(\Omega_{1}\right), \frac{\partial u}{\partial \vec{n}}=0 \text { em } \Gamma\right\}
$$

e para $u \in \mathscr{D}\left(A_{0}\right)$,

$$
\begin{aligned}
A_{0} u & =\left(-\operatorname{div}\left(p_{0}(x) \nabla u\right)+\lambda u\right) \chi_{\Omega_{1}} \\
& +\sum_{i=1}^{m}\left(\frac{1}{\left|\Omega_{0, i}\right|} \int_{\Gamma_{0, i}} p_{0}(x) \frac{\partial u}{\partial \vec{n}} d x+\int_{\Omega_{0, i}} \lambda u_{\Omega_{0, i}}\right) \chi_{\Omega_{0, i}} .
\end{aligned}
$$

Teorema 2.10. O operador $A_{0}: \mathscr{D}\left(A_{0}\right) \subset L_{\Omega_{0}}^{2}(\Omega) \rightarrow L_{\Omega_{0}}^{2}(\Omega)$ é setorial.

É fácil ver que a estimativa setorial obtida no Teorema 2.8 continua válida para $\varepsilon=0$, com o mesmo valor de $M$. Desta forma, podemos condensar os Teoremas 2.8 e 2.10 na seguinte condição de estabilidade:

(E) Para cada $\varepsilon \in\left[0, \varepsilon_{0}\right]$, existem constantes $0<\phi<\frac{\pi}{2}$ e $M_{E} \geqslant 1$ de tal maneira que $\Sigma_{-\lambda, \phi}=$ $\{\mu \in \mathbb{C}:|\arg (\mu+\lambda)|<\pi-\phi\} \subset \rho\left(-A_{\varepsilon}\right) e$

$$
\left\|\left(\mu+A_{\varepsilon}\right)^{-1}\right\|_{\mathscr{L}(X)} \leqslant \frac{M_{E}}{|\mu+\lambda|}, \quad \text { para todo } \mu \in \Sigma_{-\lambda, \phi} .
$$




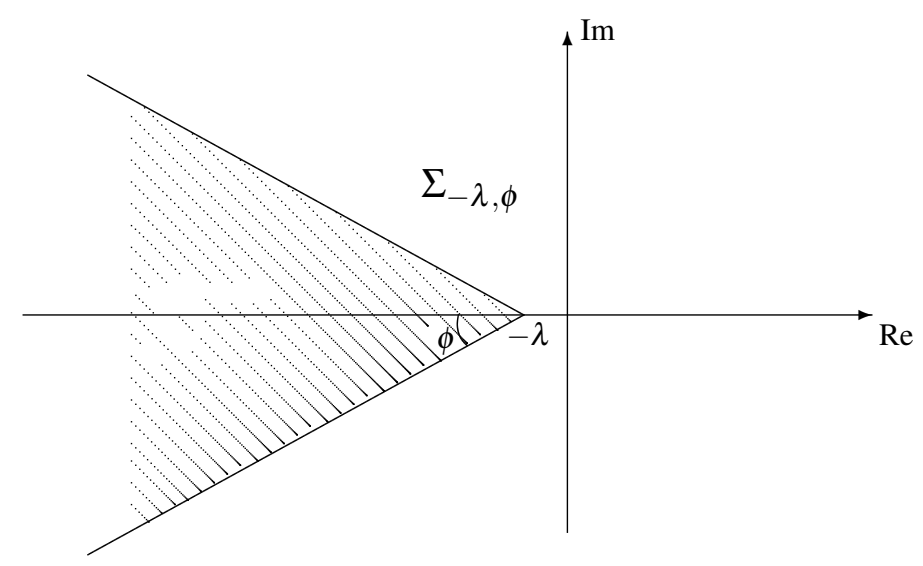

Figura 1: Setor $\Sigma_{-\lambda, \phi}$

Observemos que os resultados acima nos permitem definir os espaços de potências fracionárias $X_{\mathcal{\varepsilon}}^{\alpha}:=\mathscr{D}(A)_{\mathcal{\varepsilon}}^{\alpha}$ dos operadores $A_{\varepsilon}, \varepsilon \in\left[0, \varepsilon_{0}\right]$, e $0 \leqslant \alpha \leqslant 1$. Tais espaços verificam $X_{\varepsilon}^{\alpha} \subset H^{2 \alpha}(\Omega)$ e, em particular, $X_{\mathcal{\varepsilon}}^{\frac{1}{2}}=H^{1}(\Omega)$, munido do produto interno

$$
\langle\phi, \psi\rangle_{X_{\varepsilon}^{\frac{1}{2}}}=\int_{\Omega} p_{\varepsilon}(x) \nabla \phi \nabla \psi d x+\int_{\Omega} \lambda \phi \psi d x
$$

e $X_{0}^{\frac{1}{2}}=H_{\Omega_{0}}^{1}(\Omega)$, com o produto interno

$$
\langle\phi, \psi\rangle_{X_{0}^{\frac{1}{2}}}=\int_{\Omega_{1}} p_{0}(x) \nabla \phi \nabla \psi d x+\int_{\Omega} \lambda \phi \psi d x .
$$

Para simplificar, passaremos a denotar por $\langle,\rangle_{-\frac{1}{2}, \frac{1}{2}}$ o produto de dualidade entre $X_{\varepsilon}^{-\frac{1}{2}} \mathrm{e}$ $X_{\mathcal{\varepsilon}}^{\frac{1}{2}}$ e por $\langle,\rangle_{-s, s}$ o produto de dualidade entre $X_{\mathcal{\varepsilon}}^{-\frac{s}{2}}$ e $X_{\mathcal{\varepsilon}}^{\frac{s}{2}}, \varepsilon \in\left[0, \varepsilon_{0}\right]$.

Assim, definimos a não-linearidade $h: X_{\mathcal{\varepsilon}}^{\frac{1}{2}} \rightarrow X_{\mathcal{\varepsilon}}^{-\frac{s}{2}}$ de (2.7) e (2.9) por $h:=f_{\Omega}+g_{\Gamma}$, no sentido que

$$
\left\langle f_{\Omega}(u)+g_{\Gamma}(u), \phi\right\rangle_{-s, s}=\int_{\Omega} f(u) \phi d x+\int_{\Gamma} g(\gamma(u)) \gamma(\phi) d x,
$$

para funções $\phi \in X^{\frac{s}{2}}$.

Além disso, os operadores $-A_{\varepsilon}, \varepsilon \in\left[0, \varepsilon_{0}\right]$, geram semigrupos analíticos $\left\{e^{-A_{\varepsilon} t}: t \geqslant 0\right\}$ em $X_{\mathcal{\varepsilon}}^{\alpha}$, para os quais temos a seguinte estimativa:

$$
\left\|e^{-A_{\varepsilon} t}\right\|_{\mathscr{L}\left(X_{\varepsilon}^{\beta}, X_{\varepsilon}^{\alpha}\right)} \leqslant M_{\omega} t^{\beta-\alpha} e^{-\omega t}, \omega<\lambda, \text { para todo } t>0
$$


onde a constante $M_{\omega}$ independe de $\varepsilon$.

Ademais, se $u^{\varepsilon}\left(t, u_{0}^{\varepsilon}\right)$ denota uma solução de (2.6), e $u^{0}\left(t, u_{0}^{0}\right)$ representa uma solução de (2.8), podemos definir, para cada $\varepsilon \in\left[0, \varepsilon_{0}\right]$, um $C_{0}$-semigrupo não-linear $T_{\mathcal{\varepsilon}}(t): X_{\mathcal{\varepsilon}}^{\alpha} \rightarrow X_{\mathcal{\varepsilon}}^{\alpha}$ através da relação

$$
T_{\varepsilon}(t) u_{0}^{\varepsilon}:=u^{\varepsilon}\left(t, u_{0}^{\varepsilon}\right)
$$

Com o intuito de simplificar, denotaremos a solução de (2.8) apenas por $u\left(t, u_{0}\right)$.

Para garantirmos que o semigrupo gerado pela equação (2.8) possui um atrator global, basta assumirmos a condição de dissipatividade

( $\left.\mathbf{D}_{0}\right)$ Assumamos que a condição $(\mathbf{S})$ esteja satisfeita e sejam $C_{0}$ e $B_{0}$ as constantes de (S). Assumamos que seja positivo o primeiro autovalor, $\mu_{1}$ do problema limite

$$
\begin{cases}-\operatorname{div}\left(p_{0}(x) \nabla u\right)+\left(\lambda+C_{0}\right) u=\mu u & \text { em } \Omega_{1} \\ u_{\mid \Omega_{0, i}}:=u_{\Omega_{0, i}} & \text { em } \Omega_{0, i}, i=1, \ldots, m \\ \frac{1}{\left|\Omega_{0, i}\right|} \int_{\Gamma_{0, i}} p_{0}(x) \frac{\partial u}{\partial \vec{n}} d x+\int_{\Omega_{0, i}}\left(\lambda+C_{0}\right) u_{\Omega_{0, i}} d x=\mu\left(u_{\Omega_{0, i}}\right) & i=1, \ldots, m \\ p_{0}(x) \frac{\partial u}{\partial \vec{n}}+B_{0} u=0 & \text { em } \Gamma .\end{cases}
$$

e utilizarmos os resultados obtidos na seção precedente.

A existência de um atrator global para o problema perturbado (2.6) seguirá da convergência dos autovalores do problema perturbado para os autovalores do problema limite, que estudaremos no próximo capítulo. De fato, se o primeiro autovalor do problema limite (2.12) é positivo, a convergência de autovalores implicará que, para $\varepsilon>0$ suficientemente pequeno, o primeiro autovalor do problema perturbado será também positivo. Isto nos dará uma condição de dissipatividade sobre o problema perturbado. 


\section{Capítulo}

\section{Teoria linear}

O comportamento espectral dos operadores lineares desempenha um papel fundamental na análise das propriedades de continuidade da dinâmica não-linear. Reservamos então este capítulo para estudar o comportamento da parte linear dos operadores. Na primeira seção provamos a convergência compacta dos operadores resolventes com o objetivo de usá-la como ferramenta para analisarmos a convergência espectral dos operadores lineares. Na Seção 3.2 estudamos o comportamento do espectro dos operadores $A_{\varepsilon}, \varepsilon \in\left[0, \varepsilon_{0}\right]$. Mais precisamente, obtemos condições necessárias e suficientes para garantir que os autovalores e as autofunções de $A_{\varepsilon}$ convirjam para os autovalores e autofunções do operador $A_{0}$ quando $\varepsilon \rightarrow 0$. Este fato está provado em Rodríguez-Bernal [18], mas neste trabalho é uma conseqüência direta da convergência compacta dos operadores resolventes. Na Seção 3.3, utilizamos os resultados obtidos sobre convergência espectral dos operadores lineares para mostrar um teorema de aproximação do tipo Trotter-Kato para os semigrupos lineares. Este caminho para obter a continuidade dos semigrupos lineares foi usado por Arrieta e Carvalho [2].

Primeiramente vamos considerar os problemas de autovalores

$$
\begin{cases}-\operatorname{div}\left(p_{\varepsilon}(x) \nabla u^{\varepsilon}\right)+\lambda u^{\varepsilon}=\mu u^{\varepsilon} & \text { em } \Omega \\ p_{\varepsilon}(x) \frac{\partial u^{\varepsilon}}{\partial \vec{n}}=0 & \text { em } \Gamma .\end{cases}
$$

e o problema limite

$$
\begin{cases}-\operatorname{div}\left(p_{0}(x) \nabla u\right)+\lambda u=\mu u & \text { em } \Omega_{1} \\ \frac{1}{\left|\Omega_{0, i}\right|} \int_{\Gamma_{0, i}} p_{0}(x) \frac{\partial u}{\partial \vec{n}} d x+\int_{\Omega_{0, i}} \lambda u_{\Omega_{0, i}} d x=\mu u_{\Omega_{0, i}} & i=1, \ldots, m \\ p_{0}(x) \frac{\partial u}{\partial \vec{n}}=0 & \text { em } \Gamma .\end{cases}
$$


Utilizando a notação introduzida anteriormente, queremos mostrar que $A_{\mathcal{\varepsilon}}^{-1} \rightarrow A_{0}^{-1}$ compactamente quando $\varepsilon \rightarrow 0$. Deste fato seguirá a convergência dos autovalores e autofunções associadas a estes operadores. Para tanto, vamos estudar as propriedades de convergência das soluções do problema

$$
\begin{cases}-\operatorname{div}\left(p_{\varepsilon}(x) \nabla u^{\varepsilon}\right)+\lambda u^{\varepsilon}=h^{\varepsilon} & \text { em } \Omega \\ p_{\varepsilon}(x) \frac{\partial u^{\varepsilon}}{\partial \vec{n}}=0 & \text { em } \Gamma\end{cases}
$$

para a solução do problema limite

$$
\begin{cases}-\operatorname{div}\left(p_{0}(x) \nabla u\right)+\lambda u=h_{0} & \text { em } \Omega_{1} \\ \frac{1}{\left|\Omega_{0, i}\right|} \int_{\Gamma_{0, i}} p_{0}(x) \frac{\partial u}{\partial \vec{n}} d x+\int_{\Omega_{0, i}} \lambda u_{\Omega_{0, i}} d x=h_{\Omega_{0, i}} & i=1, \ldots, m \\ p_{0}(x) \frac{\partial u}{\partial \vec{n}}=0 & \text { em } \Gamma\end{cases}
$$

sempre que $X_{\varepsilon} \ni h_{\varepsilon} \stackrel{\varepsilon \rightarrow 0}{\longrightarrow} h_{0} \in X_{0}$ em $X$.

Vejamos algumas definições:

Dizemos que uma seqüência $\left\{u^{\varepsilon}\right\} \operatorname{com} u^{\varepsilon} \in X_{\mathcal{\varepsilon}}^{\frac{1}{2}}, X_{\varepsilon}^{\frac{1}{2}}-$ converge para $u \in X_{0}^{\frac{1}{2}}$ quando $\varepsilon \rightarrow 0$ se $\left\|u^{\varepsilon}-u\right\|_{X_{\varepsilon}^{\frac{1}{2}}} \stackrel{\varepsilon \rightarrow 0}{\longrightarrow} 0$ e dizemos que $\left\{u^{\varepsilon}\right\}$ converge $X_{\varepsilon}^{\frac{1}{2}}$-fracamente para $u \in X_{0}^{\frac{1}{2}}$ quando $\varepsilon \rightarrow 0$ se

$$
\int_{\Omega} p_{\varepsilon}(x) \nabla u^{\varepsilon} \nabla \phi d x+\int_{\Omega} \lambda u^{\varepsilon} \phi d x \rightarrow \int_{\Omega_{1}} p_{0}(x) \nabla u \nabla \phi d x+\int_{\Omega} \lambda u \phi d x, \text { para toda } \phi \in X_{0}^{\frac{1}{2}} .
$$

Notemos que $\left\{u^{\varepsilon}\right\} X_{\varepsilon}^{\frac{1}{2}}$-converge para $u \in X_{0}^{\frac{1}{2}}$ quando $\varepsilon \rightarrow 0$ se, e somente se,

$$
\int_{\Omega_{1}} p_{\varepsilon}(x)\left|\nabla u^{\varepsilon}-\nabla u\right|^{2} d x+\int_{\Omega} \lambda\left(u^{\varepsilon}-u\right)^{2} d x \stackrel{\varepsilon \rightarrow 0}{\longrightarrow} 0 \quad \text { e } \quad \int_{\Omega_{0}} p_{\varepsilon}(x)\left|\nabla u^{\varepsilon}\right|^{2} d x \stackrel{\varepsilon \rightarrow 0}{\longrightarrow} 0 .
$$

Denotamos por $X_{\mathcal{\varepsilon}}^{-\frac{1}{2}}$ o espaço dual de $X_{\mathcal{\varepsilon}}^{\frac{1}{2}}, 0 \leqslant \varepsilon \leqslant \varepsilon_{0}$. Como $X_{0}^{\frac{1}{2}}$ é um subespaço fechado de $X_{\varepsilon}^{\frac{1}{2}}$, dada $h_{\varepsilon} \in X_{\mathcal{\varepsilon}}^{-\frac{1}{2}}$, temos que $h_{\varepsilon}$ é um funcional linear limitado em $X_{\mathcal{\varepsilon}}^{\frac{1}{2}}$ e sua restrição a $X_{0}^{\frac{1}{2}}$, que também denotamos por $h_{\varepsilon}$, é um elemento de $X_{0}^{-\frac{1}{2}}$.

Dadas seqüências $\left\{\varepsilon_{n}\right\} \rightarrow 0,\left\{h_{\varepsilon_{n}}\right\}, h_{\varepsilon_{n}} \in X_{\varepsilon_{n}}^{-\frac{1}{2}}, n \in \mathbb{N}$, e $h_{0} \in X_{0}^{-\frac{1}{2}}$, dizemos que $h_{\varepsilon_{n}}$ converge para $h_{0}$ e denotamos $h_{\varepsilon_{n}} \rightarrow h_{0}$ se $\left\|h_{\varepsilon_{n}}-h_{0}\right\|_{X_{0}^{-\frac{1}{2}}} \stackrel{n \rightarrow \infty}{\longrightarrow} 0$ e $h_{\varepsilon_{n}}\left(u_{\varepsilon_{n}}\right) \rightarrow h_{0}(u)$ sempre que $u_{\varepsilon_{n}}$ $X_{\varepsilon_{n}}^{\frac{1}{2}}$-converge para $u$. 


\subsection{Convergência compacta do resolvente}

O resultado seguinte está destinado a mostrar que a família de soluções de (3.3) converge para uma solução do problema limite (3.4).

Teorema 3.1. Sejam $\left\{\varepsilon_{n}\right\}$ uma seqüência em $\left(0, \varepsilon_{0}\right]$ que converge para zero, $\left\{h_{\varepsilon_{n}}\right\}, h_{\varepsilon_{n}} \in$ $X_{\varepsilon_{n}}^{-\frac{1}{2}}, n \in \mathbb{N}$, de tal maneira que $\left\|h_{\varepsilon_{n}}\right\|_{X_{\varepsilon_{n}}^{-\frac{1}{2}}} \leqslant 1$ para todo $n \in \mathbb{N} e\left\{u_{\varepsilon_{n}}\right\}$ satisfazendo $A_{\varepsilon_{n}} u_{\varepsilon_{n}}=h_{\varepsilon_{n}}$. Então existe uma subseqüência de $\left\{u_{\varepsilon_{n}}\right\}$, que denotamos também por $\left\{u_{\varepsilon_{n}}\right\}$, e $u \in X_{0}^{\frac{1}{2}}$ de tal modo que $\left\{u_{\varepsilon_{n}}\right\}$ converge para $u X_{\varepsilon}^{\frac{1}{2}}$-fracamente e fortemente em $X$. Além disso, se $\left\{h_{\varepsilon_{n}}\right\} \subset$ $X_{\mathcal{\varepsilon}}^{-\frac{1}{2}}$ converge $X_{\mathcal{\varepsilon}}^{-\frac{1}{2}}$-fracamente para $h \in X_{0}^{-\frac{1}{2}}$, então $\left\{u_{\varepsilon_{n}}\right\}$ converge para $u X_{\mathcal{\varepsilon}}^{\frac{1}{2}}$-fracamente e fortemente em $X$, onde u é a solução de

$$
A_{0} u=h
$$

Demonstração: Notemos inicialmente que, se $\left\|h_{\varepsilon_{n}}\right\|_{X_{\varepsilon_{n}}^{-\frac{1}{2}}} \leqslant 1$, então existe uma constante $C_{1}$ de tal maneira que $\left\|h_{\varepsilon_{n}}\right\|_{X_{0}^{-\frac{1}{2}}} \leqslant C_{1}$. Como $X_{0}^{-\frac{1}{2}}$ é reflexivo, existem uma subseqüência, que denotaremos também por $\left\{h_{\varepsilon_{n}}\right\}$, e $h_{0} \in X_{0}^{-\frac{1}{2}}$ de tal maneira que $\left\langle h_{\varepsilon_{n}}, \phi_{0}\right\rangle_{-\frac{1}{2}, \frac{1}{2}} \stackrel{n \rightarrow \infty}{\longrightarrow}\left\langle h_{0}, \phi_{0}\right\rangle_{-\frac{1}{2}, \frac{1}{2}}$, para toda $\phi_{0} \in X_{0}^{\frac{1}{2}}$. Além disso,

$$
\begin{aligned}
\left|\left\langle h_{\varepsilon_{n}}, \phi_{\varepsilon_{n}}\right\rangle_{-\frac{1}{2}, \frac{1}{2}}-\left\langle h_{0}, \phi_{0}\right\rangle_{-\frac{1}{2}, \frac{1}{2}}\right| & \leqslant\left|\left\langle h_{\varepsilon_{n}}, \phi_{\varepsilon_{n}}\right\rangle_{-\frac{1}{2}, \frac{1}{2}}-\left\langle h_{\varepsilon_{n}}, \phi_{0}\right\rangle_{-\frac{1}{2}, \frac{1}{2}}\right|+\left|\left\langle h_{\varepsilon_{n}}, \phi_{0}\right\rangle_{-\frac{1}{2}, \frac{1}{2}}-\left\langle h_{0}, \phi_{0}\right\rangle_{-\frac{1}{2}, \frac{1}{2}}\right| \\
& \leqslant\left\|h_{\varepsilon_{n}}\right\|_{X_{\varepsilon_{n}}^{-\frac{1}{2}}}\left\|\phi_{\varepsilon_{n}}-\phi_{0}\right\|_{X_{\varepsilon_{n}}^{\frac{1}{2}}+o(1)}
\end{aligned}
$$

quando $n \rightarrow \infty$, ou seja, $\left\langle h_{\varepsilon_{n}}, \phi_{\varepsilon_{n}}\right\rangle_{-\frac{1}{2}, \frac{1}{2}} \rightarrow\left\langle h_{0}, \phi_{0}\right\rangle_{-\frac{1}{2}, \frac{1}{2}}$ sempre que $\left\{\phi_{\varepsilon_{n}}\right\} X_{\varepsilon_{n}}^{\frac{1}{2}}$-converge para $\phi_{0}$ quando $n \rightarrow \infty$.

Observemos ainda que

$$
\left\|\left.u_{\varepsilon_{n}}\right|_{X_{\varepsilon_{n}}^{\frac{1}{2}}} ^{2}=\int_{\Omega} p_{\varepsilon_{n}}(x)\left|\nabla u_{\varepsilon_{n}}\right|^{2} d x+\lambda \int_{\Omega}\left|u_{\varepsilon_{n}}\right|^{2} d x \leqslant\right\| h_{\varepsilon_{n}}\left\|_{X_{\varepsilon_{n}}^{-\frac{1}{2}}}\right\| u_{\varepsilon_{n}} \|_{X_{\varepsilon_{n}}^{\frac{1}{2}}},
$$

de modo que $\left\|u_{\varepsilon_{n}}\right\|_{X_{\varepsilon_{n}}^{\frac{1}{2}}} \leqslant 1$. Ainda, como $0<m_{0} \leqslant p_{\varepsilon}(x)$, para todo $x \in \Omega$ e para todo $0<\varepsilon \leqslant \varepsilon_{0}$, então

$$
m_{0} \int_{\Omega}\left|\nabla u_{\varepsilon_{n}}\right|^{2} d x+\lambda \int_{\Omega}\left|u_{\varepsilon_{n}}\right|^{2} d x \leqslant \|\left. u_{\varepsilon_{n}}\right|_{X_{\varepsilon_{n}}^{\frac{1}{2}}} ^{2} \leqslant 1
$$

ou seja, $\left\{\left\|u_{\varepsilon_{n}}\right\|_{H^{1}(\Omega)}\right\}$ é limitada. Logo, existem uma subseqüência $\left\{u_{\varepsilon_{n_{k}}}\right\}$, que denotaremos simplesmente por $\left\{u_{\varepsilon_{n}}\right\}$, e $u \in H^{1}(\Omega)$ de tal forma que $u_{\varepsilon_{n}} \stackrel{n \rightarrow \infty}{\longrightarrow} u$ fracamente em $H^{1}(\Omega) \mathrm{e}$ 
fortemente em $L^{2}(\Omega)$. Igualmente, segue de (3.5) que

$$
m_{0} \int_{K}\left|\nabla u_{\varepsilon_{n}}\right|^{2} d x+\lambda \int_{K}\left|u_{\varepsilon_{n}}\right|^{2} d x \leqslant 1
$$

para todo subconjunto aberto $K \operatorname{com} K \subset \subset \Omega$ e, sendo $H^{1}(K)$ reflexivo, podemos assumir que $u_{\varepsilon_{n}} \stackrel{n \rightarrow \infty}{\longrightarrow} u$ fracamente em $H^{1}(K)$. Logo, $\int_{K}|\nabla u|^{2} d x \leqslant \liminf _{n \rightarrow \infty} \int_{K}\left|\nabla u_{\varepsilon_{n}}\right|^{2} d x$. Por outro lado, existe uma constante $C_{2}>0$ de tal modo que

$$
\inf _{x \in K}\left\{p_{\varepsilon_{n}}(x)\right\} \int_{K}\left|\nabla u_{\varepsilon_{n}}\right|^{2} d x \leqslant \int_{K} p_{\varepsilon_{n}}(x)\left|\nabla u_{\varepsilon_{n}}\right|^{2} d x \leqslant C_{2},
$$

e, como $p_{\varepsilon_{n}}(x) \stackrel{n \rightarrow \infty}{\longrightarrow} \infty$ sobre subconjuntos compactos de $\Omega_{0}$, temos que $\left.\lim _{n \rightarrow \infty} \int\left|\nabla u_{K}\right|_{\varepsilon_{n}}\right|^{2}=0$. Temos ainda que $u$ é constante em $K$, pois $\int_{K}|\nabla u|^{2} \leqslant \liminf _{n \rightarrow \infty} \int_{K}\left|\nabla u_{\varepsilon_{n}}\right|^{2} \rightarrow 0$. Agora, como $\Omega_{0}=\bigcup_{\substack{i=1 \\ K_{i} \subset \subset \Omega}}^{\infty} K_{i}$, segue que $u \in X_{0}^{\frac{1}{2}}$.

Suponhamos agora que $X_{\varepsilon_{n}}^{\frac{1}{2}} \ni \phi_{\varepsilon_{n}} X_{\varepsilon_{n}}^{\frac{1}{2}}-$ convirja para $\phi_{0} \in X_{0}^{\frac{1}{2}}$. Neste caso, $\phi_{\varepsilon_{n}}$ converge para $\phi_{0}$ em $X$ e, como $u_{\varepsilon_{n}} \rightarrow u$ em $X$, segue que $\int_{\Omega} u_{\varepsilon_{n}} \phi_{\varepsilon_{n}} d x \stackrel{n \rightarrow \infty}{\longrightarrow} \int_{\Omega} u \phi_{0} d x$. Logo,

$$
\int_{\Omega_{1}} p_{\varepsilon_{n}}(x) \nabla u_{\varepsilon_{n}} \nabla \phi_{\varepsilon_{n}} d x+\int_{\Omega} \lambda u_{\varepsilon_{n}} \phi_{\varepsilon_{n}} d x \stackrel{n \rightarrow \infty}{\longrightarrow} \int_{\Omega_{1}} p_{0}(x) \nabla u \nabla \phi_{0} d x+\int_{\Omega} \lambda u \phi_{0} d x .
$$

Desta forma, se $\phi \in X_{0}^{\frac{1}{2}}$,

$$
\begin{aligned}
\int_{\Omega} p_{\varepsilon_{n}}(x) \nabla u_{\varepsilon_{n}} \nabla \phi d x+\int_{\Omega} \lambda u_{\varepsilon_{n}} \phi d x & =\int_{\Omega_{1}} p_{\varepsilon_{n}}(x) \nabla u_{\varepsilon_{n}} \nabla \phi d x+\int_{\Omega} \lambda u_{\varepsilon_{n}} \phi d x \\
& \rightarrow \int_{\Omega_{1}} p_{0}(x) \nabla u \nabla \phi d x+\int_{\Omega} \lambda u \phi d x,
\end{aligned}
$$

e, de $A_{\varepsilon_{n}} u_{\varepsilon_{n}}=h_{\varepsilon_{n}}$ e da convergência $\left\langle h_{\varepsilon_{n}}, \phi\right\rangle_{-\frac{1}{2}, \frac{1}{2}} \rightarrow\left\langle h_{0}, \phi\right\rangle$, segue que $\left\langle A_{0} u, \phi\right\rangle_{-\frac{1}{2}, \frac{1}{2}}=\left\langle h_{0}, \phi\right\rangle_{-\frac{1}{2}, \frac{1}{2}}$, para toda $\phi \in X_{0}^{\frac{1}{2}}$, ou seja,

$$
\left\langle A_{0} u, \phi\right\rangle_{-\frac{1}{2}, \frac{1}{2}}=\int_{\Omega_{1}} p_{0}(x) \nabla u \nabla \phi d x+\int_{\Omega} \lambda u \phi d x=\left\langle h_{0}, \phi\right\rangle_{-\frac{1}{2}, \frac{1}{2}} .
$$

Para $h_{0} \in X_{0}^{-\frac{1}{2}}$, temos

$$
0=\int_{\Omega_{1}} p_{0}(x) \nabla u \nabla \phi d x+\int_{\Omega_{1}} \lambda u \phi d x-\int_{\Omega_{1}} h_{0} \phi d x+\int_{\Omega_{0}} \lambda u \phi d x-\int_{\Omega_{0}} h_{0} \phi d x
$$


para toda $\phi \in X_{0}^{\frac{1}{2}}$. Ademais, se $\phi \in C_{c}^{1}\left(\Omega_{1}\right) \subset X_{0}^{\frac{1}{2}}$, então $p u_{x_{j}} \in H^{1}\left(\Omega_{1}\right), 1 \leqslant j \leqslant N$, e

$$
\begin{aligned}
0 & =\int_{\Omega_{1}} p_{0}(x) \nabla u \nabla \phi+\int_{\Omega_{1}} \lambda u \phi d x-\int_{\Omega_{1}} h_{0} \phi d x \\
& =-\int_{\Omega_{1}} \operatorname{div}\left(p_{0}(x) \nabla u\right) \phi d x+\int_{\Omega_{1}} \lambda u \phi d x-\int_{\Omega_{1}} h_{0} \phi d x .
\end{aligned}
$$

Segue portanto que

$$
-\operatorname{div}\left(p_{0}(x) \nabla u\right)+\lambda u=h_{0} \text { quase sempre em } \Omega_{1}
$$

e além disso, supondo por um momento que $m=1$, usando (3.7) e integrando por partes em (3.6), obtemos

$$
0=\int_{\Gamma_{0}} p_{0}(x) \phi \frac{\partial u}{\partial \vec{n}} d x+\int_{\Omega_{0}} \lambda u \phi d x-\int_{\Omega_{0}} h_{0} \phi d x=\phi_{\Omega_{0}}\left(\int_{\Gamma_{0}} p_{0}(x) \frac{\partial u}{\partial \vec{n}} d x+\left|\Omega_{0}\right| \lambda u_{\Omega_{0}}-\left|\Omega_{0}\right| h_{\Omega_{0}}\right)
$$

e

$$
\frac{1}{\left|\Omega_{0}\right|} \int_{\Gamma_{0}} p_{0}(x) \frac{\partial u}{\partial \vec{n}} d x+\lambda u_{\Omega_{0}}=h_{\Omega_{0}}
$$

Introduzimos a seguir um pouco da terminologia que será utilizada adiante.

Suponhamos que $Y$ seja um espaço de Banach e $Y_{0}$ um subespaço fechado de $Y$. Dizemos que a família de operadores $\left\{S_{\varepsilon} \in \mathscr{L}(Y): \varepsilon \in(0,1]\right\}$ converge para $S \in \mathscr{L}\left(Y_{0}\right)$ quando $\varepsilon \rightarrow 0$ se $S_{\varepsilon} v_{\varepsilon} \stackrel{\varepsilon \rightarrow 0}{\longrightarrow} S v$ em $Y$, sempre que $Y \ni v_{\varepsilon} \stackrel{\varepsilon \rightarrow 0}{\longrightarrow} v \in Y_{0}$ em $Y$, e denotamos esta convergência por $S_{\varepsilon} \stackrel{\varepsilon \rightarrow 0}{\longrightarrow} S$.

Observemos que a convergência acima não representa a convergência forte de operadores, uma vez que o vetor limite deve estar necessariamente em um subespaço específico $Y_{0}$ de $Y$. Situações mais gerais podem ser vistas em Carvalho e Piskarev [9].

Sejam $Y$ um espaço de Banach e $Y_{0}$ um subespaço fechado de $Y$. Uma família de operadores $\left\{S_{\varepsilon} \in \mathscr{L}(Y): \varepsilon \in(0,1]\right\}$ é $Y_{0}$-coletivamente compacta se cada operador $S_{\varepsilon}$ é compacto, $\varepsilon \in(0,1]$, e, para toda família limitada $\left\{v_{\varepsilon} \in Y: \varepsilon \in(0,1]\right\}$, existe uma subseqüência $\varepsilon_{n} \rightarrow 0$ tal que $\left\{S_{\varepsilon_{n}} v_{\varepsilon_{n}}\right\}$ converge para um elemento de $Y_{0}$. Dizemos que uma família de operadores $\left\{S_{\varepsilon} \in \mathscr{L}(Y): \varepsilon \in(0,1]\right\}$ converge $Y_{0^{-}}$compactamente para $S \in \mathscr{L}\left(Y_{0}\right)$ se a família é $Y_{0^{-}}$ coletivamente compacta e $S_{\varepsilon} \stackrel{\varepsilon \rightarrow 0}{\longrightarrow} S$. 
Caso não haja dúvida quanto ao espaço $Y_{0}$, utilizaremos simplesmente os termos família coletivamente compacta e convergência compacta para referirmo-nos aos termos definidos anteriormente.

Um dos resultados necessários para obtermos a continuidade do espectro e a continuidade uniforme do operador resolvente é a convergência compacta $A_{\varepsilon}{ }^{-1} \rightarrow A_{0}{ }^{-1}$ quando $\varepsilon \rightarrow 0$. Prosseguimos então com este resultado.

Teorema 3.2. A família $\left\{A_{\mathcal{\varepsilon}}^{-1}: X \rightarrow X, 0<\varepsilon \leqslant \varepsilon_{0}\right\}$ converge compactamente para o opera$\operatorname{dor} A_{0}^{-1}: X_{0} \rightarrow X_{0}$ quando $\varepsilon \rightarrow 0$.

Demonstração. Seja $\left\{h_{\varepsilon}\right\} \subset X$ tal que $h_{\mathcal{\varepsilon}} \rightarrow h \in X_{0}$ em $X$. Então $h_{\varepsilon} \rightarrow h$ em $X_{\mathcal{\varepsilon}}^{-\frac{1}{2}}$ e $\left\{h_{\varepsilon}\right\}_{X_{\varepsilon}^{-\frac{1}{2}}}$ é limitada. Como $A_{\varepsilon}: X_{\mathcal{\varepsilon}}^{\frac{1}{2}} \rightarrow X_{\varepsilon}^{-\frac{1}{2}}$ é isomorfismo, temos $A_{\varepsilon}{ }^{-1} h_{\varepsilon}:=u^{\varepsilon} \in X_{\mathcal{\varepsilon}}^{\frac{1}{2}}$. Além disso, pelo Teorema 3.1, existe $u \in X_{0}^{\frac{1}{2}}$ de tal forma que $\left\{u^{\varepsilon}\right\}$ converge para $u X_{\varepsilon}^{\frac{1}{2}}$-fracamente e fortemente em $X$. Logo,

$$
A_{\varepsilon}{ }^{-1} h_{\varepsilon} \stackrel{\varepsilon \rightarrow 0}{\longrightarrow} A_{0}{ }^{-1} h \mathrm{em} X
$$

o que nos dá a convergência $A_{\varepsilon} \stackrel{-1}{\stackrel{\varepsilon \rightarrow 0}{\longrightarrow}} A_{0}^{-1}$.

Provemos agora que a família $\left\{A_{\mathcal{\varepsilon}}{ }^{-1}: X \rightarrow X, 0<\varepsilon \leqslant \varepsilon_{0}\right\}$ é coletivamente compacta. Claramente, para cada $0<\varepsilon \leqslant \varepsilon_{0}$ fixado, o operador $A_{\varepsilon}{ }^{-1}: X \rightarrow X$ é compacto, já que $A_{\varepsilon}{ }^{-1}: X_{\varepsilon}^{\frac{1}{2}} \rightarrow$ $X_{\varepsilon}^{-\frac{1}{2}}$ é isomorfismo e $X_{\varepsilon}^{\frac{1}{2}} \hookrightarrow X$ compactamente. Seja agora $\left\{h_{\varepsilon}\right\}$ uma família limitada em $X$. Então $\left\{h_{\varepsilon}\right\}_{X_{\varepsilon}^{-\frac{1}{2}}}$ é limitada e, se $v^{\varepsilon}$ é tal que $A_{\varepsilon} v^{\varepsilon}=h_{\varepsilon}$, temos

$$
\int_{\Omega} p_{\varepsilon}(x)\left|\nabla v^{\varepsilon}\right|^{2} d x+\int_{\Omega} \lambda\left|v^{\varepsilon}\right|^{2} d x=\left\langle h_{\varepsilon}, v^{\varepsilon}\right\rangle_{-\frac{1}{2}, \frac{1}{2}} \leqslant\left\|h_{\mathcal{\varepsilon}}\right\|_{X_{\mathcal{\varepsilon}}^{-\frac{1}{2}}}\left\|v^{\varepsilon}\right\|_{X_{\varepsilon}^{\frac{1}{2}}},
$$

e $\left\{v^{\varepsilon}\right\}_{X_{\varepsilon}^{\frac{1}{2}}}$ é limitada e possui uma subseqüência convergente em $X$ para $u \in X_{0}^{\frac{1}{2}}$, completando a demonstração.

Lema 3.3. Se $S_{\varepsilon}$ converge compactamente para $S$, então existe uma constante $M>0$ de tal forma que $\left\|S_{\varepsilon}\right\|_{\mathscr{L}(Y)} \leqslant M$, para todo $\varepsilon \in\left(0, \varepsilon_{0}\right]$.

Demonstração. Suponhamos que $\left\|S_{\mathcal{\varepsilon}}\right\|_{\mathscr{L}(Y)}$ não seja limitada. Então existem seqüências $\varepsilon_{n} \stackrel{n \rightarrow \infty}{\longrightarrow}$ 0 e $\left\{u_{n}\right\} \subset Y$ de tal forma que $\left\|S_{\varepsilon_{n}} u_{n}\right\|_{Y} \stackrel{n \rightarrow \infty}{\longrightarrow} \infty$. Como $S_{\varepsilon}$ converge compactamente para $S$ quando $\varepsilon \rightarrow 0$, existem uma subseqüência, que denotaremos também por $\left\{S_{\varepsilon_{n}} u_{n}\right\}$, e $u \in Y_{0}$ tais que $S_{\varepsilon_{n}} u_{n} \rightarrow u$ quando $n \rightarrow \infty$, uma contradição. 
Lema 3.4. Suponhamos que $\left\{S_{\varepsilon} \in \mathscr{L}(Y): \varepsilon \in(0,1]\right\}$ convirja compactamente para $S$ quando $\varepsilon \rightarrow 0$ e que, além disso, $\mathscr{N}(I+S)=\{0\}$. Então existem constantes $\varepsilon_{0}>0$ e $M>0$ tais que

$$
\left\|\left(I+S_{\mathcal{E}}\right)^{-1}\right\|_{\mathscr{L}(Y)} \leqslant M, \quad \text { para todo } \varepsilon \in\left(0, \varepsilon_{0}\right]
$$

Demonstração. Devemos mostrar primeiramente que existe um $\varepsilon_{0}$ tal que $\mathscr{N}\left(I+S_{\varepsilon}\right)=\{0\}$, todo $\varepsilon \in\left(0, \varepsilon_{0}\right]$. Suponhamos, por absurdo, que existam seqüências $\varepsilon_{n} \rightarrow 0$ e $\left\{u_{n}\right\} \subset Y, u_{n} \in$ $\mathscr{N}\left(I+S_{\varepsilon_{n}}\right), n \in \mathbb{N}$, com $\left\|u_{n}\right\|_{Y}=1$. Como $S_{\varepsilon}$ converge compactamente para $S$ quando $\varepsilon \rightarrow 0$, existem uma subseqüência, que denotaremos também por $\left\{S_{\varepsilon_{n}} u_{n}\right\}$, e $u \in Y_{0}$ tais que $S_{\varepsilon_{n}} u_{n} \rightarrow u$ quando $n \rightarrow \infty$. Logo, $0=u_{n}+S_{\varepsilon_{n}} u_{n} \rightarrow u$ e $u_{n} \rightarrow-u$, e portanto $-(I+S) u=0$, com $\|u\|_{Y}=1$, o que contradiz a hipótese.

Notemos ainda que mostrar (3.8) é equivalente a mostrar que $\left\|\left(I+S_{\varepsilon}\right) u^{\varepsilon}\right\|_{Y} \geqslant \frac{1}{M}$, para todo $\varepsilon \in\left(0, \varepsilon_{0}\right]$ para todo $u^{\varepsilon} \in Y$ com $\left\|u^{\varepsilon}\right\|_{Y}=1$. Suponhamos então que isto não seja válido, ou seja, suponhamos que existam seqüências $\varepsilon_{n} \rightarrow 0$ e $\left\{u_{n}\right\}$, com $u_{n} \in Y,\left\|u_{n}\right\|_{Y}=1$, de tal maneira que $\left\|\left(I+S_{\varepsilon_{n}}\right) u_{n}\right\| \rightarrow 0$. Denotemos também por $\left\{S_{\varepsilon_{n}} u_{n}\right\}$ a subseqüência convergente de $\left\{S_{\varepsilon_{n}} u_{n}\right\}$, e suponhamos que $S_{\varepsilon_{n}} u_{n} \rightarrow u \in Y_{0},\|u\|_{Y}=1$. Então $u_{n}+S_{\varepsilon_{n}} \rightarrow 0$ e $u_{n} \rightarrow-u$. Logo, $(I+S) u=0$, uma contradição, pois $\|u\|_{Y}=1$. Logo, a estimativa em (3.8) é válida.

Lema 3.5. Para todo $\mu \in \rho\left(-A_{0}\right)$, existem $\varepsilon_{\mu}$ tal que $\mu \in \rho\left(-A_{\varepsilon}\right)$, para todo $\varepsilon \in\left(0, \varepsilon_{\mu}\right]$, e uma constante $M_{\mu}>0$ tais que

$$
\left\|\left(\mu+A_{\varepsilon}\right)^{-1}\right\|_{\mathscr{L}(X)} \leqslant M_{\mu}, \quad \text { para todo } \varepsilon \in\left(0, \varepsilon_{\mu}\right] .
$$

Além disso, $\left(\mu+A_{\varepsilon}\right)^{-1}$ converge compactamente para $\left(\mu+A_{0}\right)^{-1}$ quando $\varepsilon \rightarrow 0$.

Demonstração. Seja $\mu \in \rho\left(-A_{0}\right)$. Então, a igualdade $\left(\mu+A_{0}\right)=A_{0}\left(I+\mu A_{0}{ }^{-1}\right)$ implica a existência de $\left(I+\mu A_{0}{ }^{-1}\right)^{-1}$. Pelo Lema 3.4, existe $\varepsilon_{\mu} \in\left(0, \varepsilon_{0}\right]$ tal que $\left(I+\mu A_{\varepsilon}{ }^{-1}\right)^{-1}$ está bem definido, para todo $\varepsilon \in\left(0, \varepsilon_{\mu}\right]$, e é uniformemente limitado. $\operatorname{Logo},\left(\mu+A_{\varepsilon}\right)^{-1}=(I+$ $\left.\mu A_{\varepsilon}{ }^{-1}\right)^{-1} A_{\varepsilon}{ }^{-1}$ existe. Como $A_{\varepsilon}{ }^{-1}$ converge compactamente para $A_{0}{ }^{-1}$ quando $\varepsilon \rightarrow 0$, temos que $\left\{\left\|A_{\mathcal{\varepsilon}}^{-1}\right\|_{\mathscr{L}(X)}: \varepsilon \in\left(0, \varepsilon_{\mu}\right]\right\}$ é limitado. Assim, a limitação de $\left\{\left(I+\mu A_{\varepsilon}{ }^{-1}\right)^{-1}: 0<\varepsilon \leqslant\right.$ $\left.\varepsilon_{\mu}\right\}$ nos garante que $\left(\mu+A_{\varepsilon}\right)^{-1}$ converge compactamente para $\left(\mu+A_{0}\right)^{-1}$ quando $\varepsilon \rightarrow 0$.

\subsection{Continuidade do espectro}

Nesta seção estudamos as propriedades de convergência espectral dos operadores lineares. O resultado seguinte nos diz que o espectro de $A_{\varepsilon}$, para $\varepsilon$ pequeno, aproxima-se do espectro de $A_{0}$. 
Como os operadores $A_{\varepsilon}, \varepsilon \in\left[0, \varepsilon_{0}\right]$, são auto-adjuntos e têm resolvente compacto, os espectros $\sigma\left(-A_{\varepsilon}\right), \varepsilon \in\left[0, \varepsilon_{0}\right]$, consistem apenas de autovalores reais e isolados.

Teorema 3.6. As seguintes afirmações são válidas:

(i) Para todo $\mu_{0} \in \sigma\left(-A_{0}\right)$, existem seqüências $\varepsilon_{n} \rightarrow 0 e\left\{\mu_{n}\right\}, \mu_{n} \in \sigma\left(-A_{\varepsilon_{n}}\right), n \in \mathbb{N}$, tais que $\mu_{n} \rightarrow \mu_{0}$ quando $n \rightarrow \infty$.

(ii) Se para alguma seqüência $\varepsilon_{n} \rightarrow 0$, temos que $\mu_{n} \rightarrow \mu_{0}$ quando $n \rightarrow \infty$, com $\mu_{n} \in \sigma\left(-A_{\varepsilon_{n}}\right)$, $n \in \mathbb{N}$, então $\mu_{0} \in \sigma\left(-A_{0}\right)$.

Demonstração. (i) Seja $\mu_{0} \in \sigma\left(-A_{0}\right)$ e consideremos $U=U\left(\mu_{0}, \delta\right)=\left\{\mu \in \mathbb{C}:\left|\mu-\mu_{0}\right| \leqslant \delta\right\}$, sendo $\delta$ escolhido de tal forma que $U \cap \sigma\left(-A_{0}\right)=\left\{\mu_{0}\right\}$. Nosso objetivo é mostrar que existe um $\bar{\varepsilon} \in\left(0, \varepsilon_{0}\right]$ tal que $U \cap \sigma\left(-A_{\varepsilon}\right) \neq \varnothing$, para $\varepsilon \in(0, \bar{\varepsilon}]$.

Mostremos primeiramente que existe $\bar{\varepsilon}>0$ tal que $\left\|\left(\mu+A_{\varepsilon}\right)^{-1}\right\|_{\mathscr{L}(X)}$ é limitado para $\varepsilon \in$ $(0, \bar{\varepsilon}]$ e $\mu \in \partial U$. Para tanto, é suficiente provarmos que $\left\|\left(I+\mu A_{\varepsilon}{ }^{-1}\right)^{-1}\right\|_{\mathscr{L}(X)}$ é limitado, para quaisquer $\varepsilon \in(0, \bar{\varepsilon}], \mu \in \partial U$, já que $\left\|A_{\varepsilon}{ }^{-1}\right\|_{\mathscr{L}(X)}$ é limitado para $\varepsilon \in\left(0, \varepsilon_{0}\right]$. Suponhamos, por absurdo, que existam seqüências $\left\{\mu_{n}\right\} \subset \partial U, \mu_{n} \rightarrow \bar{\mu} \in \partial U,\left\{u_{n}\right\} \subset X,\left\|u_{n}\right\|_{X}=1$, e $\varepsilon_{n} \rightarrow 0$ tais que

$$
\left\|\left(I+\mu_{n} A_{\varepsilon_{n}}^{-1}\right) u_{n}\right\|_{X} \rightarrow 0 \quad \text { quando } n \rightarrow \infty
$$

Observemos agora que

$$
\mu_{n} A_{\varepsilon_{n}}^{-1} u_{n}=\left(\mu_{n}-\bar{\mu}\right) A_{\mathcal{\varepsilon}_{n}}^{-1} u_{n}+\bar{\mu} A_{\mathcal{\varepsilon}_{n}}^{-1} u_{n} \rightarrow v
$$

uma vez que $A_{\varepsilon_{n}}^{-1} u_{n}$ é relativamente compacto. Assim, como $u_{n}+\mu_{n} A_{\varepsilon_{n}}^{-1} u_{n} \rightarrow 0$, obtemos que $u_{n} \rightarrow-v$ e $\|v\|_{X}=1$. Ainda, segue que $u_{n}+\mu_{n} A_{\varepsilon_{n}}^{-1} u_{n} \rightarrow-\left(I+\bar{\mu} A_{0}{ }^{-1}\right) v$ e $\left(I+\bar{\mu} A_{0}{ }^{-1}\right) v=$ $0,\|v\|_{X}=1$, uma contradição, pois $\bar{\mu} \in \rho\left(-A_{0}\right)$.

Suponhamos, novamente por redução ao absurdo, que $U \subset \rho\left(-A_{\varepsilon}\right)$, qualquer que seja $\varepsilon \in$ $(0, \bar{\varepsilon}]$. Como a função $\mu \mapsto\left(I+\mu A_{\varepsilon}{ }^{-1}\right)^{-1}$ é holomorfa para todo $\mu \in \partial U$, segue do que fizemos anteriormente e do Teorema do Máximo Módulo que

$$
\left\|\left(I+\mu_{0} A_{\varepsilon}{ }^{-1}\right)^{-1}\right\|_{\mathscr{L}(X)} \leqslant \max _{\substack{\left|\mu-\mu_{0}\right|=\delta \\ \varepsilon \in\left(0, \varepsilon_{0}\right]}}\left\|\left(I+\mu_{0} A_{\varepsilon}{ }^{-1}\right)^{-1}\right\|_{\mathscr{L}(X)}=C_{1}<\infty
$$

para alguma constante $C_{1}>0$. Logo, para $u \in X$ e $\varepsilon_{n} \rightarrow 0$, obtemos

$$
\|u\|_{X}=\left\|\left(I+\mu_{0} A_{\varepsilon_{n}}^{-1}\right)^{-1}\left(I+\mu_{0} A_{\varepsilon_{n}}^{-1}\right) u\right\|_{X} \leqslant C_{2}\left\|\left(I+\mu_{0} A_{\varepsilon_{n}}^{-1}\right) u\right\|_{X},
$$


de maneira que $\left\|\left(I+\mu_{0} A_{0}^{-1}\right) u\right\|_{X} \geqslant \frac{1}{C_{2}}\|u\|_{X}$ e $\mu_{0} \in \rho\left(-A_{0}\right)$, uma contradição. Portanto, $U\left(\mu_{0}, \delta\right)$ contém algum ponto de $\sigma\left(-A_{\varepsilon}\right)$, para $\varepsilon$ apropriadamente pequeno.

(ii) Consideremos as seqüências $\varepsilon_{n} \rightarrow 0$ e $\left\{\mu_{n}\right\}, \mu_{n} \in \sigma\left(-A_{\varepsilon_{n}}\right)$, tais que $\mu_{n} \rightarrow \mu_{0}$ quando $n \rightarrow \infty$. Seja $\left\{u_{n}\right\} \subset X$, com $\left\|u_{n}\right\|_{X}=1$ e $\left\|\left(I+\mu_{n} A_{\varepsilon_{n}}^{-1}\right) u_{n}\right\|_{X}=0$. Então

$$
\left\|\left(I+\mu_{0} A_{\varepsilon_{n}}^{-1}\right) u_{n}\right\|_{X} \leqslant\left\|\left(I+\mu_{n} A_{\varepsilon_{n}}^{-1}\right) u_{n}\right\|_{X}+\left\|\left(\mu-\mu_{n}\right) A_{\varepsilon_{n}}^{-1} u_{n}\right\|_{X} \rightarrow 0
$$

quando $n \rightarrow \infty$. Como $\left\{A_{\mathcal{E}_{n}}^{-1}: n \in \mathbb{N}\right\}$ é coletivamente compacta e $\left\|u_{n}\right\|_{X}=1$, tomando subsequiências se necessário, resulta que $\mu_{0}\left(A_{\varepsilon_{n}}\right)^{-1} u_{n} \rightarrow v$ e $u_{n} \rightarrow-v,\|v\|_{X}=1$. Portanto, $(I+$ $\left.\mu_{0} A_{0}{ }^{-1}\right) v=0,\|v\|_{X}=1$, o que significa que $\mu \in \sigma\left(-A_{0}\right)$.

O seguinte resultado trata da convergência uniforme dos operadores resolventes sobre determinados conjuntos limitados de $X$.

Lema 3.7. Seja $K$ um subconjunto compacto de $\rho\left(-A_{0}\right)$. Então existe uma constante $\varepsilon_{K}>0$ de tal forma que $K \subset \rho\left(-A_{\varepsilon}\right)$ para todo $\varepsilon \in\left(0, \varepsilon_{K}\right] e$

$$
\sup _{\alpha \in[0,1]} \sup _{\varepsilon \in\left(0, \varepsilon_{K}\right]} \sup _{\mu \in K}\left\|\left(\mu+A_{\varepsilon}\right)^{-1}\right\|_{\mathscr{L}\left(X, X_{\varepsilon}^{\alpha}\right)}<\infty
$$

Se $f_{\Omega} \in L^{2}(\Omega), g_{\Gamma} \in L^{2}(\Gamma) e h=f_{\Omega}+g_{\Gamma} \in X_{0}^{-\frac{s}{2}}$ é definida por

$$
\langle h, \phi\rangle_{-s, s}=\int_{\Omega} f_{\Omega} \phi+\int_{\Gamma} g_{\Gamma} \phi, \quad \forall \phi \in X_{0}^{\frac{s}{2}}
$$

então estendemos $h$ a $X_{\mathcal{\varepsilon}}^{-\frac{s}{2}}$ de maneira natural, e obtemos

$$
\sup _{\mu \in K}\left\|\left(\mu+A_{\varepsilon}\right)^{-1} h-\left(\mu+A_{0}\right)^{-1} h\right\|_{X_{\varepsilon}^{\frac{1}{2}}} \stackrel{\varepsilon \rightarrow 0}{\longrightarrow} 0
$$

Além disso, se J é um subconjunto de $X_{0}^{-\frac{s}{2}}$ constituído por elementos da forma $f_{\Omega}+g_{\Gamma}$, com $f_{\Omega}$ (respectivamente $g_{\Gamma}$ ) variando em um subconjunto limitado de $L^{2}(\Omega)\left(\right.$ respectivamente $L^{2}(\Gamma)$ ), temos

$$
\sup _{\substack{\mu \in K \\ h \in J}}\left\|\left(\mu+A_{\varepsilon}\right)^{-1} h-\left(\mu+A_{0}\right)^{-1} h\right\|_{X_{\varepsilon}^{\frac{1}{2}}} \stackrel{\varepsilon \rightarrow 0}{\longrightarrow} 0 .
$$

Demonstração. Vamos primeiramente provar que existe $\varepsilon_{K}>0$ tal que $K \subset \rho\left(-A_{\varepsilon}\right)$ para todo $\varepsilon \in\left(0, \varepsilon_{K}\right]$. Suponhamos, para tanto, que existam sequiências $\varepsilon_{n} \rightarrow 0,\left\{\mu_{n}\right\} \subset K$ tais que $\mu_{n}$ é autovalor de $-A_{\varepsilon_{n}}$. Como $K$ é compacto, podemos assumir que existe $\bar{\mu} \in K$ de tal forma que $\mu_{n} \rightarrow \bar{\mu}$. Segue do Teorema 3.6, item (ii), que $\bar{\mu} \in \sigma\left(-A_{0}\right)$, o que é uma contradição. Para 
provarmos (3.9), assumamos que existam sequiências $\varepsilon_{n} \rightarrow 0,\left\{\mu_{n}\right\} \subset K, \mu_{n} \rightarrow \bar{\mu} \in K$ de tal maneira que

$$
\left\|\left(I+\mu_{n} A_{\varepsilon_{n}}^{-1}\right)^{-1}\right\|_{\mathscr{L}(X)} \rightarrow \infty .
$$

Como $\mu_{n} A_{\varepsilon_{n}}^{-1}$ converge compactamente para $\bar{\mu} A_{0}{ }^{-1}$, isto contradiz o Lema 3.4. Logo,

$$
\sup _{\substack{\mu \in K \\ \varepsilon \in\left(0, \varepsilon_{k}\right]}}\left\|\left(I+\mu A_{\varepsilon}{ }^{-1}\right)^{-1}\right\|_{\mathscr{L}(X)}<\infty .
$$

Além disso, da Desigualdade do Momento, segue a existência de uma constante $C_{1}$ de tal modo que, para quaisquer $0<\alpha<1, u \in X$, temos

$$
\begin{aligned}
\left\|A_{\varepsilon}^{\alpha}\left(\mu+A_{\varepsilon}\right)^{-1} u\right\|_{X} & \leqslant C_{1}\left\|A_{\varepsilon}\left(\mu+A_{\varepsilon}\right)^{-1} u\right\|_{X}^{\alpha}\left\|\left(\mu+A_{\varepsilon}\right)^{-1} u\right\|_{X}^{1-\alpha} \\
& \left.\leqslant C_{1} \|\left(I-\mu\left(\mu+A_{\varepsilon}\right)^{-1}\right) u\right)\left\|_{X}^{\alpha}\right\|\left(\mu+A_{\varepsilon}\right)^{-1} u \|_{X}^{1-\alpha}
\end{aligned}
$$

provando (3.9).

Fazemos a prova de (3.11) do seguinte modo: sejam $u^{\varepsilon}=\left(\mu+A_{\varepsilon}\right)^{-1} h$ e $u=\left(\mu+A_{0}\right)^{-1} h$. Então

$$
\begin{gathered}
\int_{\Omega} p_{\varepsilon}(x)\left|\nabla u^{\varepsilon}\right|^{2} d x+(\lambda+\mu) \int_{\Omega}\left(u^{\varepsilon}\right)^{2} d x=\left\langle h, u^{\varepsilon}\right\rangle_{-s, s} \\
\int_{\Omega_{1}} p_{\varepsilon}(x) \nabla u^{\varepsilon} \nabla u d x+(\lambda+\mu) \int_{\Omega} u^{\varepsilon} u d x=\langle h, u\rangle_{-s, s} \\
\int_{\Omega_{1}} p_{0}(x)|\nabla u|^{2} d x+(\lambda+\mu) \int_{\Omega} u^{2} d x=\langle h, u\rangle_{-s, s} .
\end{gathered}
$$

Assim,

$$
\begin{aligned}
\left\langle h, u^{\varepsilon}-u\right\rangle_{-s, s} & =\int_{\Omega} p_{\varepsilon}(x)\left|\nabla u^{\varepsilon}\right|^{2} d x-\int_{\Omega_{1}} p_{0}(x)|\nabla u|^{2} d x+(\lambda+\mu) \int_{\Omega}\left(u^{\varepsilon}\right)^{2} d x-(\lambda+\mu) \int_{\Omega} u^{2} d x \\
& =\int_{\Omega} p_{\varepsilon}(x)\left|\nabla u^{\varepsilon}-\nabla u\right|^{2} d x+(\lambda+\mu) \int_{\Omega}\left(u^{\varepsilon}-u\right)^{2} d x-\int_{\Omega_{1}}\left(p_{\varepsilon}(x)-p_{0}(x)\right)|\nabla u|^{2} d x \\
& +2 \int_{\Omega_{1}} p_{\varepsilon}(x) \nabla u^{\varepsilon} \nabla u d x+2(\lambda+\mu) \int_{\Omega} u^{\varepsilon} u d x-2 \int_{\Omega_{1}} p_{0}(x)|\nabla u|^{2} d x-2(\lambda+\mu) \int_{\Omega} u^{2} d x \\
& =\int_{\Omega} p_{\varepsilon}(x)\left|\nabla u^{\varepsilon}-\nabla u\right|^{2} d x+(\lambda+\mu) \int_{\Omega}\left(u^{\varepsilon}-u\right)^{2} d x-\int_{\Omega_{1}}\left(p_{\varepsilon}(x)-p_{0}(x)\right)|\nabla u|^{2} d x .
\end{aligned}
$$

Segue facilmente do Teorema 3.1 que $u^{\varepsilon} \rightarrow u X_{\varepsilon}^{\frac{1}{2}}$-fracamente. Logo, $\left\langle h, u^{\varepsilon}-u\right\rangle_{-\frac{1}{2}, \frac{1}{2}} \rightarrow 0$ e portanto $\left\langle h, u^{\varepsilon}-u\right\rangle_{-s, s} \rightarrow 0$. Além disso, como $p_{\varepsilon} \rightarrow p_{0}$ uniformemente em $\Omega_{1}$ e $\int_{\Omega_{1}}|\nabla u|^{2}<\infty$, da expressão acima concluímos que $u^{\varepsilon} X_{\varepsilon}^{\frac{1}{2}}$-converge para $u$. 
Consideremos agora $J$ um subconjunto limitado de $X_{0}^{-\frac{s}{2}}$ dado da seguinte forma: existem $M_{1}$ e $M_{2}$ tais que, se $h \in J$, então

$$
h=f_{\Omega}+g_{\Gamma}
$$

com $\left\|f_{\Omega}\right\|_{L^{2}(\Omega)} \leqslant M_{1}$ e $\left\|g_{\Gamma}\right\|_{L^{2}(\Gamma)} \leqslant M_{2}$. Denotemos por $J_{\mathcal{E}}$ o conjunto das extensões naturais $h_{\varepsilon}: X_{\varepsilon}^{\frac{s}{2}} \rightarrow \mathbb{R}$ de elementos $h: X_{0}^{\frac{s}{2}} \rightarrow \mathbb{R}$ de $J$ à $X_{\varepsilon}^{\frac{s}{2}}$. É fácil ver que

$$
\sup _{\varepsilon \in\left(0, \varepsilon_{0}\right]} \sup _{h_{\varepsilon} \in J_{\varepsilon}}\left\|h_{\varepsilon}\right\|_{X_{\varepsilon}^{-\frac{s}{2}}}<\infty
$$

Segue do fato que $\sup _{\varepsilon \in\left(0, \varepsilon_{0}\right]}\left\|u^{\varepsilon}-u\right\|_{X_{\varepsilon}^{\frac{1}{2}}}<\infty$, do fato que $\left\|u^{\varepsilon}-u\right\|_{X} \rightarrow 0$ quando $\varepsilon \rightarrow 0$ e da Desigualdade do Momento que $\left\|u^{\varepsilon}-u\right\|_{X_{\varepsilon}^{\frac{s}{2}}} \rightarrow 0$ quando $\varepsilon \rightarrow 0$. Isto garante que $\sup _{h \in J}\left\langle h, u^{\varepsilon}-\right.$ $u\rangle_{-s, s} \rightarrow 0$ quando $\varepsilon \rightarrow 0$. O resultado agora segue da identidade

$$
\left\langle h, u^{\varepsilon}-u\right\rangle_{-s, s}=\int_{\Omega} p_{\varepsilon}(x)\left|\nabla u^{\varepsilon}-\nabla u\right|^{2} d x+(\lambda+\mu) \int_{\Omega}\left(u^{\varepsilon}-u\right)^{2} d x-\int_{\Omega_{1}}\left(p_{\varepsilon}(x)-p_{0}(x)\right)|\nabla u|^{2} d x .
$$

Sejam $\mu$ um ponto isolado de $\sigma\left(-A_{0}\right)$ e $\delta>0$ tais que $\{z \in \mathbb{C}:|z-\mu| \leqslant \delta\} \cap \sigma\left(-A_{0}\right)=$ $\{\mu\}$. Definimos o auto-espaço generalizado associado a $\mu$ como

$$
W\left(\mu,-A_{0}\right)=Q_{0}(\mu) X
$$

onde

$$
Q_{0}(\mu)=\frac{1}{2 \pi i} \int_{|z-\mu|=\delta}\left(z+A_{0}\right)^{-1} d z
$$

Como $K=\{z \in \mathbb{C}:|z-\mu|=\delta\}$ é compacto e $K \subset \rho\left(-A_{0}\right)$, então, pelo lema anterior, existe um $\varepsilon_{K}$ tal que $K \subset \rho\left(-A_{\mathcal{\varepsilon}}\right)$, para $\varepsilon \in\left(0, \varepsilon_{K}\right]$. Para $\varepsilon \in\left(0, \varepsilon_{K}\right]$, o auto-espaço generalizado $W\left(\mu,-A_{\varepsilon}\right)=Q_{\varepsilon}(\mu) X$, onde

$$
Q_{\varepsilon}(\mu)=\frac{1}{2 \pi i} \int_{|z-\mu|=\delta}\left(z+A_{\varepsilon}\right)^{-1} d z
$$

Acerca das projeções $Q_{\varepsilon}$ e $Q_{0}$, temos os seguinte resultado:

Lema 3.8. Qualquer que seja $\mu \in\{z \in \mathbb{C}:|z-\mu|=\delta\}, Q_{\varepsilon}(\mu)$ converge compactamente para $Q_{0}(\mu)$ quando $\varepsilon \rightarrow 0$. 
Demonstração. Como $\left(\mu+A_{\varepsilon}\right)^{-1} \stackrel{\varepsilon \rightarrow 0}{\longrightarrow}\left(\mu+A_{0}\right)^{-1}$, para todo $\mu$ tal que $\left|\mu-\mu_{0}\right|=\delta$, e

$$
\left\{\left\|\left(\mu+A_{\varepsilon}\right)^{-1}\right\|_{\mathscr{L}(X)}: 0<\varepsilon \leqslant \varepsilon_{0}\right\}
$$

é limitado, segue do Teorema da Convergência Dominada que

$$
Q_{\varepsilon}\left(\mu_{0}\right)=\frac{1}{2 \pi i} \int_{\left|\mu-\mu_{0}\right|=\delta}\left(\mu+A_{\varepsilon}\right)^{-1} d \mu \stackrel{\varepsilon \rightarrow 0}{\longrightarrow} \frac{1}{2 \pi i} \int_{\left|\mu-\mu_{0}\right|=\delta}\left(\mu+A_{\varepsilon}\right)^{-1} d \mu=Q_{0}\left(\mu_{0}\right) .
$$

Podemos escrever

$$
Q_{\varepsilon}\left(\mu_{0}\right)=A_{\varepsilon}^{-1} \frac{1}{2 \pi i} \int_{\left|z-\mu_{0}\right|=\delta}\left(I+z A_{\varepsilon}{ }^{-1}\right)^{-1} d z .
$$

Como, do Lema (3.5), $\left\|I+z A_{\varepsilon}{ }^{-1}\right\|_{\mathscr{L}(X)}^{-1}$ é uniformemente limitado, temos

$$
\frac{1}{2 \pi i} \int_{\left|z-\mu_{0}\right|=\delta}\left(I+z A_{\varepsilon}{ }^{-1}\right)^{-1} d z
$$

é uniformemente limitada, e também, como $A_{\mathcal{\varepsilon}}{ }^{-1} \rightarrow A_{0}{ }^{-1}$ compactamente quando $\varepsilon \rightarrow 0$, resulta que $Q_{\varepsilon}\left(\mu_{0}\right)$ converge compactamente para $Q_{0}\left(\mu_{0}\right)$ quando $\varepsilon \rightarrow 0$.

Teorema 3.9. As seguintes afirmações são válidas:

(i) Existe $\varepsilon_{0}>0$ tal que $\operatorname{dim} W\left(\mu,-A_{\varepsilon}\right)=\operatorname{dim} W\left(\mu,-A_{0}\right)$, para todo $0<\varepsilon \leqslant \varepsilon_{0}$.

(ii) Para todo $u \in W\left(\mu_{0},-A_{0}\right)$, existe uma seqüência $\left\{u^{\varepsilon}\right\}, u^{\varepsilon} \in W\left(\mu_{0},-A_{\varepsilon}\right)$, tal que $u^{\varepsilon} \stackrel{\varepsilon \rightarrow 0}{\longrightarrow} u$.

(iii) Para toda seqüência $\varepsilon_{n} \rightarrow 0$, a seqüência $\left\{u_{n}\right\}, u_{n} \in W\left(\mu,-A_{\varepsilon_{n}}\right), n \in \mathbb{N}$, com $\left\|u_{n}\right\|_{X}=1$, possui uma subseqüência convergente e todo ponto limite desta seqüência pertence ao auto-espaço generalizado $W\left(\mu_{0},-A_{0}\right)$.

Demonstração. (i) Mostremos primeiramente que existe $\bar{\varepsilon} \in\left(0, \varepsilon_{0}\right]$ tal que posto $\left(Q_{\varepsilon}\left(\mu_{0}\right)\right) \geqslant$ $\operatorname{posto}\left(Q_{0}\left(\mu_{0}\right)\right), \varepsilon \in(0, \bar{\varepsilon}]$. Suponhamos então que exista uma seqüência $\varepsilon_{n} \rightarrow 0$ de tal forma que posto $\left(Q_{\varepsilon_{n}}\left(\mu_{0}\right)\right)<\operatorname{posto}\left(Q_{0}\left(\mu_{0}\right)\right)$. Seja $\left\{v_{1}, \ldots, v_{k}\right\}$ uma base para $W\left(\mu_{0},-A_{0}\right)=Q_{0}\left(\mu_{0}\right) X_{0}$; então

$$
\left\{Q_{\varepsilon_{n}}\left(\mu_{0}\right) v_{1}, \cdots Q_{\varepsilon_{n}}\left(\mu_{0}\right) v_{k}\right\}
$$

é um conjunto linearmente dependente em $X$. Escolhamos $\alpha_{1}^{n}, \cdots, \alpha_{k}^{n}$ de tal forma que $\sum_{i=1}^{k}\left|\alpha_{i}^{n}\right|=$ $1 \mathrm{e}$

$$
\sum_{i=1}^{k} \alpha_{i}^{n} Q_{\varepsilon_{n}}\left(\mu_{0}\right) v_{i}=0
$$


Tomando subseqüências se necessário, podemos assumir que existem $\alpha_{1}, \cdots, \alpha_{k}$ tais que $\alpha_{i}^{n} \rightarrow$ $\alpha_{i}, 1 \leqslant i \leqslant k$. Logo,

$$
\sum_{i=1}^{k} \alpha_{i} v_{i}=\sum_{i=1}^{k} \alpha_{i} Q_{0}\left(\mu_{0}\right) v_{i}=0 \text { e } \sum_{i=1}^{k}\left|\alpha_{i}\right|=1,
$$

contradizendo a independência linear de $\left\{v_{1}, \ldots, v_{k}\right\}$. Logo, existe um $\bar{\varepsilon}$ tal que posto $\left(Q_{\varepsilon}\left(\mu_{0}\right)\right)$ $\geqslant \operatorname{posto}\left(Q_{0}\left(\mu_{0}\right)\right)$, para $\varepsilon \in(0, \bar{\varepsilon}]$.

Assumamos agora que $\operatorname{posto}\left(Q_{\varepsilon_{n}}\left(\mu_{0}\right)\right)>\operatorname{posto}\left(Q_{0}\left(\mu_{0}\right)\right)$, para alguma seqüência $\varepsilon_{n} \rightarrow 0$. Então, para cada $n \in \mathbb{N}$, existe $u_{n} \in W\left(\mu_{0},-A_{\varepsilon_{n}}\right),\left\|u_{n}\right\|_{X}=1$, tal que $\operatorname{dist}\left(u_{n}, W\left(\mu_{0}, A_{0}\right)\right)=$ $\left\|u_{n}\right\|_{X}=1$. Da convergência compacta, podemos assumir que $Q_{\varepsilon_{n}}\left(\mu_{0}\right) u_{n}=u_{n} \rightarrow Q_{0}\left(\mu_{0}\right) u_{0}=$ $u_{0}$, e assim,

$$
1 \leqslant\left\|u_{n}-Q_{0}\left(\mu_{0}\right) u_{n}\right\|_{X}=\left\|Q_{\varepsilon_{n}}\left(\mu_{0}\right) u_{n}-Q_{0}\left(\mu_{0}\right) u_{n}\right\|_{X} \rightarrow 0
$$

uma contradição.

(ii) Sejam $u \in W\left(\mu_{0},-A_{0}\right)$ e $u^{\varepsilon}=Q_{\varepsilon}\left(\mu_{0}\right) u$. Como $Q_{\varepsilon}\left(\mu_{0}\right) u \rightarrow Q_{0}\left(\mu_{0}\right) u$ e $u=Q_{0}\left(\mu_{0}\right) u$, temos que $u^{\varepsilon} \rightarrow u$.

(iii) Suponhamos que $\varepsilon_{n} \rightarrow 0$ e consideremos $\left\{u_{n}\right\} \subset X, u_{n} \in W\left(\mu_{0},-A_{\varepsilon_{n}}\right), n \in \mathbb{N},\left\|u_{n}\right\|_{X}$ $=1$. Como $Q_{\varepsilon}\left(\mu_{0}\right) \rightarrow Q_{0}\left(\mu_{0}\right)$ compactamente quando $\varepsilon \rightarrow 0$, então $\left\{Q_{\varepsilon}\left(\mu_{0}\right): \varepsilon \in\left(0, \varepsilon_{0}\right]\right\}$ é coletivamente compacta. Logo, $u_{n}=Q_{\varepsilon_{n}}\left(\mu_{0}\right) u_{n}$ possui uma subsequiência convergente, digamos, $u_{n} \rightarrow u$, ou seja, $Q_{\varepsilon_{n}}\left(\mu_{0}\right) u_{n} \rightarrow Q_{0}\left(\mu_{0}\right) u$ e $u \in W\left(\mu_{0},-A_{0}\right)$.

\subsection{Convergência dos semigrupos lineares}

A continuidade do espectro obtida na seção anterior nos permite obter estimativas sobre o comportamento dos semigrupos lineares, que serão úteis na análise da dinâmica não-linear.

As propriedades dos operadores $A_{\mathcal{\varepsilon}}, \varepsilon \in\left[0, \varepsilon_{0}\right]$, implicam, para cada $\omega<\lambda$, na existência de uma constante $M_{\omega}$, independente de $\varepsilon$, de tal modo que

$$
\left\|e^{-A_{\varepsilon} t} u\right\|_{X_{\varepsilon}^{\frac{1}{2}}} \leqslant M_{\omega} t^{-\left(\frac{1+s}{2}\right)} e^{-\omega t}\|u\|_{X_{\varepsilon}^{-\frac{s}{2}}}
$$

para todo $t>0$ e todo $\varepsilon \in\left[0, \varepsilon_{0}\right]$.

Teorema 3.10. Sejam $K$ um subconjunto compacto de $X_{0}^{-\frac{s}{2}} e 0<\theta<\frac{1}{2}$. Se $h=f_{\Omega}+g_{\Gamma} \in K$ é como em (3.10) e estendemos $h$ de forma natural a $X_{\mathcal{\varepsilon}}^{-\frac{s}{2}}$, então existe uma função $v:\left(0, \varepsilon_{0}\right] \rightarrow$ 
$\mathbb{R}^{+}, v(\varepsilon) \stackrel{\varepsilon \rightarrow 0}{\longrightarrow} 0$, de tal forma que

$$
\sup _{h \in K}\left\|e^{-A_{\varepsilon} t} h-e^{-A_{0} t} h\right\|_{X_{\varepsilon}^{\frac{1}{2}}} \leqslant v(\varepsilon) e^{-\omega t} t^{-\theta-\frac{(1+s)}{2}}, \quad \text { para todo } t>0 .
$$

Demonstração. Sejam $\delta \in(0,1)$ e $0<\theta<\frac{1}{2}$ fixados. Trabalharemos separadamente os casos $0<t<\delta$ e $t \geqslant \delta$.

Como $h$ está em um subconjunto compacto de $X_{0}^{-\frac{s}{2}}$ e $0<t<\delta$, segue que

$$
\begin{aligned}
\left\|e^{-A_{\varepsilon} t} h-e^{-A_{0} t} h\right\|_{X_{\varepsilon}^{\frac{1}{2}}} & \leqslant 2 M_{\omega} e^{-\omega t} t^{-\left(\frac{1+s}{2}\right)} \\
& \leqslant 2 M_{\omega} e^{-\omega t} t^{\theta} t^{-\theta-\left(\frac{1+s}{2}\right)} \\
& \leqslant 2 M_{\omega} e^{-\omega t} \delta^{\theta} t^{-\theta-\left(\frac{1+s}{2}\right)} .
\end{aligned}
$$

Observemos agora que, como os operadores $A_{\varepsilon}, \varepsilon \in\left[0, \varepsilon_{0}\right]$, são setoriais, os operadores $-A_{\varepsilon}$ geram semigrupos analíticos $\left\{e^{-A_{\varepsilon} t}: t \geqslant 0\right\}$, que são dados por

$$
e^{-A_{\varepsilon} t}=\frac{1}{2 \pi i} \int_{\Gamma} e^{\mu t}\left(\mu+A_{\varepsilon}\right)^{-1} d \mu, \quad \varepsilon \in\left[0, \varepsilon_{0}\right],
$$

onde $\Gamma$ é a fronteira do setor $\Sigma_{-\lambda, \phi}$, orientada de maneira que a parte imaginária de $\mu$ cresce quando $\mu$ percorre $\Gamma$. Desta forma, para $\bar{\omega}<\lambda$, temos

$$
e^{\left(-A_{\varepsilon}+\bar{\omega} I\right) t}=\frac{1}{2 \pi i} \int_{\Gamma} e^{(\mu+\bar{\omega}) t}\left(\mu+\bar{\omega}+A_{\varepsilon}-\bar{\omega}\right)^{-1} d \mu, \quad \varepsilon \in\left[0, \varepsilon_{0}\right] .
$$

Fazendo a mudança de variáveis $\mu+\bar{\omega} \mapsto \mu$, e denotando $B_{\varepsilon}:=A_{\varepsilon}-\bar{\omega} I, \varepsilon \in\left[0, \varepsilon_{0}\right]$, podemos escrever

$$
e^{-B_{\varepsilon} t}=\frac{1}{2 \pi i} \int_{\Gamma_{0}} e^{\mu t}\left(\mu+B_{\varepsilon}\right)^{-1} d \mu, \quad \varepsilon \in\left[0, \varepsilon_{0}\right],
$$

sendo $\Gamma_{0}$ a fronteira do setor $\Sigma_{0, \phi}$. Nosso objetivo é estimar, para $t \geqslant \delta$, a diferença

$$
2 \pi t^{\frac{1+s}{2}}\left\|e^{-B_{\varepsilon} t} h-e^{-B_{0} t} h\right\|_{X_{\varepsilon}^{\frac{1}{2}}}=\left\|\int_{\Gamma_{0}} t^{\frac{1+s}{2}} e^{\mu t}\left[\left(\mu+B_{\varepsilon}\right)^{-1} h-\left(\mu+B_{0}\right)^{-1} h\right] d \mu\right\|_{X_{\varepsilon}^{\frac{1}{2}}}
$$

Vejamos que a integral do lado direito em (3.14) é convergente. Para $\mu \in \Gamma_{0}$, obtivemos previamente a estimativa setorial $\left\|\left(\mu+B_{\varepsilon}\right)^{-1}\right\|_{\mathscr{L}\left(X_{\varepsilon}^{-\frac{s}{2}}\right)} \leqslant \frac{M}{|\mu|}$, para todo $\varepsilon \in\left[0, \varepsilon_{0}\right]$. Logo,

$$
\begin{aligned}
\left\|B_{\varepsilon}\left(\mu+B_{\varepsilon}\right)^{-1} h\right\|_{X_{\varepsilon}^{-\frac{s}{2}}} & =\left\|\left(I-\mu\left(\mu+B_{\varepsilon}\right)^{-1}\right) h\right\|_{X_{\varepsilon}^{-\frac{s}{2}}} \\
& \leqslant\|h\|_{X_{\varepsilon}^{-\frac{s}{2}}}+|\mu|\left\|\left(\mu+B_{\varepsilon}\right)^{-1} h\right\|_{X_{\varepsilon}^{-\frac{s}{2}}} \\
& \leqslant M_{1}\|h\|_{X_{\varepsilon}^{-\frac{s}{2}}} .
\end{aligned}
$$


Da Desigualdade do Momento $\operatorname{com} \beta=\frac{-s}{2}<\alpha=\frac{1}{2}<\gamma=1-\frac{s}{2}$, obtemos

$$
\begin{aligned}
\left\|\left(\mu+B_{\varepsilon}\right)^{-1} h\right\|_{X_{\varepsilon}^{\frac{1}{2}}} & =\left\|B_{\varepsilon^{\frac{1}{2}}}\left(\mu+B_{\varepsilon}\right)^{-1} h\right\|_{X} \\
& \leqslant C\left\|B_{\varepsilon}{ }^{\frac{1-s}{2}}\left(\mu+B_{\varepsilon}\right)^{-1} h\right\|_{X}^{\frac{1+s}{2}}\left\|B_{\varepsilon}^{-\frac{s}{2}}\left(\mu+B_{\varepsilon}\right)^{-1} h\right\|_{X}^{\frac{1-s}{2}} \\
& =C\left\|B_{\varepsilon}\left(\mu+B_{\varepsilon}\right)^{-1} h\right\|_{X_{\varepsilon}^{-\frac{s}{2}}}^{\frac{1+s}{2}}\left\|\left(\mu+B_{\varepsilon}\right)^{-1} h\right\|_{X_{\varepsilon}^{-\frac{s}{2}}}^{\frac{1-s}{2}} \\
& \leqslant C M_{1}^{\frac{1+s}{2}}\|h\|_{X_{\varepsilon}^{-\frac{s}{2}}}^{\frac{1+s}{\mid}} \frac{M^{\frac{1-s}{2}}}{|\mu|^{\frac{1-s}{2}}}\|h\|_{X_{\varepsilon}^{-\frac{s}{2}}}^{\frac{1-s}{2}} \\
& \leqslant \frac{M_{2}}{|\mu|^{\frac{1-s}{2}}\|h\|_{X_{\varepsilon}^{-\frac{s}{2}}}}
\end{aligned}
$$

e assim, $\left\|\left(\mu+B_{\varepsilon}\right)^{-1} h-\left(\mu+B_{0}\right)^{-1} h\right\|_{X_{\varepsilon}^{\frac{1}{2}}} \leqslant \frac{2 M_{2}}{|\mu|^{\frac{1-s}{2}}}\|h\|_{X_{\varepsilon}^{-\frac{s}{2}}}$.

Desta forma, temos

$$
\begin{aligned}
&\left\|\int_{\Gamma_{0}} t^{\frac{1+s}{2}} e^{\mu t}\left[\left(\mu+B_{\varepsilon}\right)^{-1} h-\left(\mu+B_{0}\right)^{-1} h\right] d \mu\right\|_{X_{\varepsilon}^{\frac{1}{2}}} \leqslant t^{\frac{1+s}{2}} \int_{\Gamma_{0}}\left|e^{\mu t}\right| \frac{2 M_{2}}{|\mu|^{\frac{1-s}{2}}}\|h\|_{X_{\varepsilon}^{-\frac{s}{2}}} d \mu \\
& \stackrel{\gamma=\mu t}{=} 2 M_{2} t^{\frac{1+s}{2}} \int_{\Gamma_{0}}\left|e^{\gamma}\left\|\left.\gamma\right|^{\frac{s-1}{2}} t^{\frac{1-s}{2}}\right\| h \|_{X_{\varepsilon}^{-\frac{s}{2}}} \frac{d \gamma}{t}\right. \\
&=M_{3}\|h\|_{X_{\varepsilon}^{-\frac{s}{2}}}
\end{aligned}
$$

Para $t \geqslant \delta$, procedemos da seguinte forma: como a integral do lado direto em (3.14) é uniformemente convergente em $\varepsilon$, dado $\eta>0$, podemos escrever $\Gamma_{0}=\Gamma_{1}^{\eta} \cup \Gamma_{2}^{\eta}$, com $\Gamma_{1}^{\eta}$ limitada e $\Gamma_{2}^{\eta}$ satisfazendo

$$
\sup _{\varepsilon \in\left(0, \varepsilon_{0}\right]} \sup _{h \in K}\left\|t^{\frac{1+s}{2}} \int_{\Gamma_{2}^{\eta}} e^{\mu t}\left[\left(\mu+B_{\varepsilon}\right)^{-1} h-\left(\mu+B_{0}\right)^{-1} h\right] d \mu\right\|_{X_{\varepsilon}^{\frac{1}{2}}}<\frac{\eta}{2} .
$$

Sobre $\Gamma_{1}^{\eta}$, fazemos a mudança de variáveis $\beta=\mu t$ em (3.14), obtendo

$$
\left\|t_{\Gamma_{1}^{\eta}}^{\frac{1+s}{2}} \int^{\beta}\left[\left(\frac{\beta}{t}+B_{\varepsilon}\right)^{-1} h-\left(\frac{\beta}{t}+B_{0}\right)^{-1}\right] \frac{d \beta}{t}\right\|_{X_{\varepsilon}^{\frac{1}{2}}} .
$$

Agora, novamente lançando mão da Desigualdade do Momento e da estimativa setorial que obtivemos, segue que

$$
\left\|\left(\frac{\beta}{t}+B_{\varepsilon}\right)^{-1}-\left(\frac{\beta}{t}+B_{0}\right)^{-1}\right\|_{X_{\varepsilon}^{\frac{1}{2}}} \leqslant 2 \frac{M^{\frac{1+s}{2}}}{\left|\frac{\beta}{t}\right|^{\frac{1+s}{2}}}\|h\|_{X}=\frac{M_{4}}{|\beta|^{\frac{1+s}{2}}} t^{\frac{1+s}{2}}\|h\|_{X},
$$


ou seja,

$$
\sup _{h \in K}\left\|\left(\frac{\beta}{t}+B_{\varepsilon}\right)^{-1} h-\left(\frac{\beta}{t}+B_{0}\right)^{-1}\right\|_{X_{\varepsilon}^{\frac{1}{2}}} \leqslant \frac{M_{5}}{|\beta|^{\frac{1+s}{2}}} t^{\frac{1+s}{2}}
$$

Também, como $t \geqslant \delta$, temos que $\frac{1}{t} \in\left[0, \frac{1}{\delta}\right] \subset \subset \mathbb{R}$ e, para $\beta \in \Gamma_{1}^{\eta}, \frac{\beta}{t}$ está em um subconjunto compacto de $\rho\left(-B_{0}\right)$. Logo, como no Lema 3.7, garantimos a existência de uma função $\tilde{v}$ : $\left(0, \varepsilon_{0}\right] \rightarrow \mathbb{R}^{+}$, com $\tilde{v}(\varepsilon) \rightarrow 0$ quando $\varepsilon \rightarrow 0$, com

$$
\sup _{h \in K}\left\|\left(\frac{\beta}{t}+B_{\varepsilon}\right)^{-1} h-\left(\frac{\beta}{t}+B_{0}\right)^{-1}\right\|_{X_{\varepsilon}^{\frac{1}{2}}} \leqslant \tilde{v}(\varepsilon), \quad \text { para } t \geqslant \delta \text { e } \beta \in \Gamma_{1}^{\eta} .
$$

Interpolando as duas últimas expressões (com $1-2 \theta$ e $2 \theta$, respectivamente), obtemos

$$
\sup _{h \in K}\left\|\left(\frac{\beta}{t}+B_{\varepsilon}\right)^{-1} h-\left(\frac{\beta}{t}+B_{0}\right)^{-1}\right\|_{X_{\varepsilon}^{\frac{1}{2}}} \leqslant \frac{M_{5}^{1-2 \theta}}{|\beta|^{\left(\frac{1+s}{2}\right)-\theta}} t^{\left(\frac{1+s}{2}\right)-\theta} \tilde{v}(\varepsilon)^{2 \theta} .
$$

Logo, existe $\bar{\varepsilon}>0$ tal que, para $\varepsilon \in(0, \bar{\varepsilon}]$, temos

$$
\begin{gathered}
\sup _{h \in K}\left\|2 \pi t^{\theta-\left(\frac{1+s}{2}\right)} \int_{\Gamma_{1}^{\eta}} e^{\beta}\left[\left(\frac{\beta}{t}+B_{\varepsilon}\right)^{-1} h-\left(\frac{\beta}{t}+B_{0}\right)^{-1} h\right] d \beta\right\|_{X_{\varepsilon}^{\frac{1}{2}}} \\
<\int_{\Gamma_{1}^{\eta}}\left|e^{\beta}\right| \frac{M_{6}}{|\beta|^{\left(\frac{1+s}{2}\right)-\theta}} \tilde{v}(\varepsilon)^{2 \theta} d|\beta| \\
<\frac{\eta}{2},
\end{gathered}
$$

isto é,

$$
\sup _{h \in K}\left\|e^{-B_{\varepsilon} t} h-e^{-B_{0} t} h\right\|_{X_{\varepsilon}^{\frac{1}{2}}} \leqslant \frac{\eta}{4 \pi} t^{-\theta-\left(\frac{1+s}{2}\right)}+\frac{\eta}{4 \pi} t^{-\theta-\left(\frac{1+s}{2}\right)}, \quad \text { para } t \geqslant \delta .
$$

Portanto, existe uma função $v:\left(0, \varepsilon_{0}\right] \rightarrow \mathbb{R}^{+}, \operatorname{com} v(\varepsilon) \stackrel{\varepsilon \rightarrow 0}{\longrightarrow} 0$, satisfazendo

$$
\sup _{h \in K}\left\|e^{-A_{\varepsilon} t} h-e^{-A_{0} t} h\right\|_{X_{\varepsilon}^{\frac{1}{2}}} \leqslant v(\varepsilon) e^{-\omega t} t^{-\left(\frac{1+s}{2}\right)-\theta} \text {, para todo } t>0 .
$$

De forma inteiramente análoga, podemos demonstrar o seguinte resultado:

Teorema 3.11. Sejam $K$ um subconjunto compacto de $X_{0}^{\frac{1}{2}}$ e $0<\theta<\frac{1}{2}$. Então existe uma função $\tilde{v}:\left(0, \varepsilon_{0}\right] \rightarrow \mathbb{R}^{+}, \tilde{v}(\varepsilon) \stackrel{\varepsilon \rightarrow 0}{\longrightarrow} 0$, de tal forma que

$$
\sup _{u \in K}\left\|e^{-A_{\varepsilon} t} u-e^{-A_{0} t} u\right\|_{X_{\varepsilon}^{\frac{1}{2}}} \leqslant \tilde{v}(\varepsilon) e^{-\omega t} t^{-\theta-\frac{1}{2}}, \quad \text { para todo } t>0 .
$$




\section{Capítulo}

\section{Continuidade dos atratores}

Neste capítulo estudamos a continuidade da família de atratores $\left\{\mathscr{A}_{\varepsilon}: 0 \in\left(0, \varepsilon_{0}\right]\right\}$ para (2.6) e $\mathscr{A}_{0}$ para (2.8) quando $\varepsilon \rightarrow 0$. Na Seção 4.1 examinamos a continuidade dos semigrupos não-lineares, que é uma conseqüência da continuidade dos resolventes e de um teorema de aproximação do tipo Trotter-Kato obtidos no capítulo anterior, e também da Fórmula da Variação das Constantes. Destes resultados segue a semicontinuidade superior dos atratores contida na Seção 4.2 .

A demonstração da semicontinuidade inferior da família de atratores requer hipóteses e resultados adicionais, além dos já obtidos nos capítulos precedentes. Mais precisamente, assumiremos na Seção 4.3.1 que todos as soluções de equilíbrio de (2.8) são hiperbólicas. Em seguida, provamos a continuidade dos conjuntos de equilíbrio. Na Seção 4.4 provamos que as variedades instáveis de $u_{j}$, para $j=1, \ldots n_{0}$ são contínuas em $X_{\varepsilon}^{\frac{1}{2}}$ quando $\varepsilon \rightarrow 0$, utilizando a convergência do semigrupos linearizados. Concluímos, na última seção, a semicontinuidade inferior e, portanto, a continuidade da família de atratores.

Antes de prosseguirmos, voltemos por um momento à questão da existência de atratores para os semigrupos associados ao problema (2.6). Notemos que o problema (2.5) é o problema limite para

$$
\begin{cases}-\operatorname{div}\left(p_{\varepsilon}(x) \nabla u^{\varepsilon}\right)+\left(\lambda+C_{0}\right) u^{\varepsilon}=\mu u^{\varepsilon} & \text { em } \Omega \\ p_{\varepsilon}(x) \frac{\partial u^{\varepsilon}}{\partial \vec{n}}+B_{0} u^{\varepsilon}=0 & \text { em } \Gamma\end{cases}
$$

Do Teorema 3.2 e da hipótese $\left(\mathbf{D}_{0}\right)$, segue que o primeiro autovalor do problema (4.1) também é positivo, para $\varepsilon$ suficientemente pequeno. Disto segue que a seguinte condição de dissipatividade $\left(\mathbf{D}_{\varepsilon}\right)$ está satisfeita: 
$\left(\mathbf{D}_{\varepsilon}\right)$ Assumamos que a condição $(\mathbf{S})$ e a condição de dissipatividade $\left(\mathbf{D}_{0}\right)$ estejam satisfeitas e sejam $C_{0}$ e $B_{0}$ as constantes de $\mathbf{( S )}$. Então existe $\bar{\varepsilon} \in\left(0, \varepsilon_{0}\right]$ de tal maneira que o primeiro autovalor, $\mu_{1}^{\varepsilon}$, do problema

$$
\begin{cases}-\operatorname{div}\left(p_{\varepsilon}(x) \nabla u^{\varepsilon}\right)+\left(\lambda+C_{0}\right) u^{\varepsilon}=\mu u & \text { em } \Omega \\ p_{\varepsilon}(x) \frac{\partial u^{\varepsilon}}{\partial \vec{n}}+B_{0} u^{\varepsilon}=0 & \text { em } \Gamma\end{cases}
$$

é positivo, para todo $\varepsilon \in(0, \bar{\varepsilon}]$.

Com isto, segue do Teorema 2.5 que os semigrupos gerados por (2.6) têm atratores globais $\mathscr{A}_{\varepsilon}$ em $X_{\mathcal{\varepsilon}}^{\frac{1}{2}}, \varepsilon \in[0, \bar{\varepsilon}]$. Além disso, temos o seguinte resultado

Teorema 4.1. Os atratores globais $\mathscr{A}_{\mathcal{\varepsilon}} \subset X_{\mathcal{\varepsilon}}^{\frac{1}{2}}$ para os semigrupos $\left\{T_{\varepsilon}(t): t \geqslant 0\right\}, \varepsilon \in[0, \bar{\varepsilon}]$, associados aos problemas (2.6) $e(2.8)$ satisfazem

$$
\sup _{\varepsilon \in\left[0, \varepsilon_{0}\right]} \sup _{w \in \mathscr{A} \mathcal{\varepsilon}}\|w\|_{X_{\varepsilon}^{\frac{1}{2}}}<\infty
$$

$e$

$$
\sup _{\varepsilon \in\left[0, \varepsilon_{0}\right]} \sup _{w \in \mathscr{A}_{\varepsilon}}\|w\|_{L^{\infty}(\Omega)}<\infty .
$$

Demonstração: A existência local de soluções segue de [4]; enquanto a existência dos atratores e limitação uniforme em $L^{\infty}(\Omega)$ seguem de [3]. Com isto, podemos assumir, sem perda de generalidade, que as não-linearidades $f$ e $g$ são globalmente Lipschitz e globalmente limitadas. Para obtermos a estimativa remanescente, procedemos de maneira usual, utilizando a Fórmula de Variação das Constantes e (2.11).

Deste ponto em diante assumimos, sem perda de generalidade, que $f$ e $g$ são globalmente Lipschitz e uniformemente limitadas com derivadas uniformemente limitadas até segunda ordem. Mais precisamente, assumiremos que existem constantes $L_{f}, L_{g}, C_{f}$ e $C_{g}$ de tal forma que

$$
\begin{gathered}
|j(s)-j(t)| \leqslant L_{j}|t-s|, \text { para quaisquer } s, t \in \mathbb{R}, j=f, g \\
\left|f^{(i)}(s)\right| \leqslant C_{f},\left|g^{(i)}(s)\right| \leqslant C_{g}, \text { para todo } s \in \mathbb{R}, i=0,1,2 .
\end{gathered}
$$

\subsection{Continuidade dos semigrupos não-lineares}

Para estudarmos a continuidade dos semigrupos não-lineares, usaremos a seguinte formulação da Desigualdade de Gronwall, de Henry [15]: 
Lema 4.2 (Desigualdade de Gronwall generalizada). Sejam $t<r, \phi:[t, r] \rightarrow \mathbb{R}^{+}$uma função contínua, $a:[t, r] \rightarrow \mathbb{R}^{+}, b>0$ e $0<\beta \leqslant 1$. Assumamos que

$$
\phi(t) \leqslant a(t)+b \int_{t}^{r}(s-t)^{\beta-1} \phi(s) d s, t \leqslant r
$$

\section{Então}

$$
\phi(t) \leqslant \sum_{k=0}^{\infty}\left(B^{k} a\right)(t)
$$

onde

$$
B^{k} a(t)=\int_{t}^{r} \frac{(b \Gamma(\beta))^{k}}{\Gamma(k \beta)}(s-t)^{k \beta-1} a(s) d s .
$$

Além disso, se a $(t)$ é constante, com $a(t) \equiv a$, então

$$
\phi(t) \leqslant a E_{\beta}\left((b \Gamma(\beta))^{\frac{1}{\beta}}\right)(s-t) \leqslant a c e^{(b \Gamma(\beta))^{\frac{1}{\beta}}(r-t)} .
$$

Se $a(t)=c_{0} \int_{t}^{r}(s-t)^{-\alpha} e^{\rho(s-r)} d s, \rho>0$, então temos

$$
\phi(t) \leqslant \frac{c_{0}}{b}\left[E_{\beta}\left((b \Gamma(\beta))^{\frac{1}{\beta}}\right)(s-t)-1\right] \leqslant \frac{c_{0}}{b}\left[e^{(b \Gamma(\beta))^{\frac{1}{\beta}}(r-t)}-1\right],
$$

e, finalmente, se $\psi:[t, r] \rightarrow \mathbb{R}^{+}$é uma função contínua e $a(t)=c_{0} \int_{t}^{r}(s-t)^{-\alpha} e^{\rho s} \psi(s) d s$, $\rho>0$, então

$$
\phi(t) \leqslant c_{0} c^{\prime} \Gamma(\beta) \int_{t}^{r}(s-t)^{\beta-1} e^{\rho s} e^{d(b \Gamma(\beta))^{\frac{1}{\beta}}(s-t)} \psi(s) d s .
$$

Demonstração: Se

$$
(B \phi)(t)=b \int_{t}^{r}(s-t)^{\beta-1} \phi(s) d s
$$

segue de (4.2) que

$$
\begin{aligned}
\phi(t) & \leqslant a(t)+(B \phi)(t) \leqslant a(t)+(B a)(t)+\left(B^{2} \phi\right)(t) \\
& \leqslant \sum_{k=0}^{n-1}\left(B^{k} a\right)(t)+\left(B^{n} \phi\right)(t) .
\end{aligned}
$$

Suponhamos, por indução, que

$$
\left(B^{n-1} \phi\right)(t)=\int_{t}^{r} \frac{(b \Gamma(\beta))^{n-1}}{\Gamma((n-1) \beta)}(s-t)^{(n-1) \beta-1} \phi(s) d s .
$$


Então

$$
\begin{aligned}
B\left(B^{n-1} \phi\right)(t) & =b \int_{t}^{r}(s-t)^{\beta-1}\left[\int_{t}^{r} \frac{(b \Gamma(\beta))^{n-1}}{\Gamma((n-1) \beta)}(\theta-t)^{(n-1) \beta-1} \phi(\theta) d \theta\right] d s \\
& =\frac{b^{n} \Gamma(\beta)^{n-1}}{\Gamma((n-1) \beta)} \int_{t}^{r} \phi(\theta)\left[\int_{t}^{\theta}(s-t)^{\beta-1}(\theta-s)^{(n-1) \beta-1} d s\right] d \theta \\
& =\frac{b^{n} \Gamma(\beta)^{n-1}}{\Gamma((n-1) \beta)} \int_{t}^{r} \phi(\theta)\left[\int_{0}^{\theta-t} u^{\beta-1}(\theta-t-u)^{(n-1) \beta-1} d u\right] d \theta .
\end{aligned}
$$

Fazendo $z=\frac{u}{\theta-t}$, obtemos

$$
\begin{aligned}
\left(B^{n} \phi\right)(t) & =\frac{b^{n} \Gamma(\beta)^{n-1}}{\Gamma((n-1) \beta)} \int_{t}^{r} \phi(\theta)(\theta-t)^{\beta}\left[\int_{0}^{1} z^{\beta-1}(\theta-t)^{(n-1) \beta-1}(1-z)^{(n-1) \beta-1}\right] d \theta \\
& =\frac{b^{n} \Gamma(\beta)^{n-1}}{\Gamma((n-1) \beta)} \int_{t}^{r} \phi(\theta)(\theta-t)^{n \beta-1} d \theta \int_{0}^{1} z^{\beta-1}(1-z)^{(n-1) \beta-1} d z .
\end{aligned}
$$

Ainda, lembrando que

$$
\int_{0}^{1} z^{\beta-1}(1-z)^{(n-1) \beta-1} d z=\mathrm{B}(\beta,(n-1) \beta)=\frac{\Gamma(\beta) \Gamma((n-1) \beta)}{\Gamma(n \beta)},
$$

obtemos

$$
\left(B^{n} \phi\right)(t)=\frac{b^{n} \Gamma(\beta)^{n}}{\Gamma(n \beta)} \int_{t}^{r} \phi(\theta)(\theta-t)^{n \beta-1} d \theta
$$

Estudemos a convergência da série $\sum_{k=0}^{\infty} \frac{z^{n}}{\Gamma(n \beta)}$. Se $a_{n}=\Gamma(n \beta)^{-1}$, então

$$
\frac{a_{n+1}}{a_{n}}=\frac{\Gamma(n \beta)}{\Gamma((n+1) \beta)}=\frac{\mathrm{B}(\beta, \mathrm{n} \beta)}{\Gamma(\beta)}=\frac{1}{\Gamma(\beta)} \int_{0}^{1} t^{\beta-1}(1-t)^{n \beta-1} d t
$$

Portanto, pelo Teorema da Convergência Dominada de Lebesgue, temos que $\frac{a_{n+1}}{a_{n}} \stackrel{n \rightarrow \infty}{\longrightarrow} 0$. Logo, $B^{n} \phi(t) \rightarrow 0$ quando $n \rightarrow \infty \mathrm{e}$

$$
\phi(t) \leqslant \sum_{k=0}^{\infty}\left(B^{k} a\right)(t) .
$$

Suponhamos agora que $a(t)$ seja constante, com $a(t) \equiv a$. Então

$$
\begin{aligned}
\left(B^{n} a\right)(t) & =\int_{t}^{r} \frac{b^{n} \Gamma(\beta)^{n}}{\Gamma(n \beta)}(s-t)^{n \beta-1} d s \\
& =\frac{a b^{n} \Gamma(\beta)^{n}}{n \beta \Gamma(n \beta)}(r-t)^{n \beta} \\
& =\frac{a b^{n} \Gamma(\beta)^{n}}{\Gamma(n \beta+1)}(r-t)^{n \beta},
\end{aligned}
$$


e assim

$$
\phi(t) \leqslant a E_{\beta}\left((b \Gamma(\beta))^{\frac{1}{\beta}}(r-t) \leqslant a c e^{(b \Gamma(\beta))^{\frac{1}{\beta}}}(r-t) .\right.
$$

$$
\begin{aligned}
& \text { Se } a(t)=c_{0} \int_{t}^{r}(s-t)^{-\alpha} e^{\rho(s-r)} d s, \operatorname{com} \alpha=1-\beta \text {, então } \\
& \begin{aligned}
\left(B^{n} a\right)(t) & =c_{0} \frac{b^{n} \Gamma(\beta)^{n}}{\Gamma(n \beta)} \int_{t}^{r}(s-t)^{n \beta-1}\left[\int_{s}^{r}(\theta-s)^{-\alpha} e^{\rho(\theta-r)} d \theta\right] d s \\
& =c_{0} \frac{b^{n} \Gamma(\beta)^{n}}{\Gamma(n \beta)} \int_{t}^{r} e^{\rho(\theta-r)}\left[\int_{t}^{\theta}(s-t)^{n \beta-1}(\theta-s)^{-\alpha} d s\right] d \theta \\
& =c_{0} \frac{b^{n} \Gamma(\beta)^{n}}{\Gamma(n \beta)} \int_{t}^{r} e^{\rho(\theta-r)}\left[\int_{t}^{\theta} u^{n \beta-1}(\theta-t-u)^{-\alpha} d s\right] d \theta .
\end{aligned}
\end{aligned}
$$

Fazendo $v=\frac{u}{\theta-t}$ temos

$$
\begin{aligned}
\left(B^{n} a\right)(t) & =c_{0} \frac{b^{n} \Gamma(\beta)^{n}}{\Gamma(n \beta)} \int_{t}^{r} e^{\rho(\theta-r)}\left[\int_{0}^{1} v^{n \beta-1}(\theta-t)^{n \beta-1}(\theta-t)^{1-\alpha}(1-v)^{-1+\beta} d v\right] d \theta \\
& =c_{0} \frac{b^{n} \Gamma(\beta)^{n}}{\Gamma(n \beta)} \int_{t}^{r} e^{\rho(\theta-r)}(\theta-t)^{(n+1) \beta-1} d \theta B(\beta, n \beta) \\
& =c_{0} \frac{b^{n} \Gamma(\beta)^{n+1}}{\Gamma((n+1) \beta)} \int_{t}^{r} e^{\rho(\theta-r)}(\theta-t)^{(n+1) \beta-1} d \theta \\
& \leqslant c_{0} \frac{b^{n} \Gamma(\beta)^{n+1}}{\Gamma((n+1) \beta)} \int_{0}^{r-t} s^{(n+1) \beta-1} d s .
\end{aligned}
$$

Assim,

$$
\begin{gathered}
\sum_{n=0}^{\infty}\left(B^{n} a\right)(t) \leqslant c_{0} \sum_{n=0}^{\infty} \frac{b^{n} \Gamma(\beta)^{n+1}}{\Gamma((n+1) \beta)} \int_{0}^{r-t} s^{(n+1) \beta-1} d s=c_{0} \sum_{n=0}^{\infty} \frac{b^{n} \Gamma(\beta)^{n+1}}{\Gamma((n+1) \beta+1)}(r-t)^{(n+1) \beta} \\
\sum_{n=0}^{\infty}\left(B^{n} a\right)(t) \leqslant \frac{c_{0}}{b} \sum_{n=0}^{\infty} \frac{b^{\frac{1}{\beta}} \Gamma(\beta)^{\frac{1}{\beta}}}{\Gamma((n+1) \beta+1)}(r-t)^{(n+1) \beta}=\frac{c_{0}}{b}\left[E_{\beta}\left(b^{\frac{1}{\beta}} \Gamma(\beta)^{\frac{1}{\beta}}(r-t)\right)-1\right] .
\end{gathered}
$$

Segue que $\phi(t) \leqslant \frac{c_{0}}{b}\left[E_{\beta}\left(b^{\frac{1}{\beta}} \Gamma(\beta)^{\frac{1}{\beta}}(r-t)\right)-1\right] \leqslant \frac{c_{0}}{b}\left[e^{b^{\frac{1}{\beta}} \Gamma(\beta)^{\frac{1}{\beta}}(r-t)}-1\right]$.

Por fim, suponhamos que $a(t)=c_{0} \int_{t}^{r}(s-t)^{-\alpha} e^{\rho s} \psi(s) d s$. Então

$$
\begin{aligned}
\left(B^{n} a\right)(t) & =c_{0} \int_{t}^{r} \frac{b^{n} \Gamma(\beta)^{n}}{\Gamma(n \beta)}(s-t)^{n \beta-1}\left[\int_{s}^{r}(\theta-s)^{-\alpha} e^{\rho \theta} \psi(\theta) d \theta\right] d s \\
& =c_{0} \int_{t}^{r} \frac{b^{n} \Gamma(\beta)^{n}}{\Gamma(n \beta)} e^{\rho \theta} \psi(\theta)\left[\int_{t}^{\theta}(s-t)^{n \beta-1}(\theta-s)^{-\alpha} d s\right] d \theta \\
& =c_{0} \int_{t}^{r} \frac{b^{n} \Gamma(\beta)^{n}}{\Gamma(n \beta)} e^{\rho \theta} \psi(\theta)(\theta-t)^{(n+1) \beta-1} \mathrm{~B}(\beta, n \beta) d \theta \\
& =c_{0} \frac{b^{n} \Gamma(\beta)^{n+1}}{\Gamma((n+1) \beta)} \int_{t}^{r} e^{\rho \theta} \psi(\theta)(\theta-t)^{(n+1) \beta-1} d \theta
\end{aligned}
$$


Assim,

$$
\begin{aligned}
\phi(t) & \leqslant \sum_{n=0}^{\infty}\left(B^{n} a\right)(t)=c_{0} \int_{t}^{r} \sum_{n=0}^{\infty} \frac{b^{n} \Gamma(\beta)^{n+1}}{\Gamma((n+1) \beta)} e^{\rho \theta} \psi(\theta)(\theta-t)^{(n+1) \beta-1} d \theta \\
& =c_{0} \Gamma(\beta) \int_{t}^{r} \sum_{n=0}^{\infty} \frac{b^{n} \Gamma(\beta)^{n+1}(\theta-t)^{n \beta}}{\Gamma((n+1) \beta)} e^{\rho \theta} \psi(\theta)(\theta-t)^{\beta-1} d \theta \\
& =c_{0} \Gamma(\beta) \int_{t}^{r} \widetilde{E}_{\beta}\left((b \Gamma(\beta))^{\frac{1}{\beta}}(\theta-t)\right) e^{\rho \theta} \psi(\theta)(\theta-t)^{\beta-1} d \theta \\
& \leqslant c_{0} c^{\prime} \Gamma(\beta) \int_{t}^{r} e^{d(b \Gamma(\beta))^{\frac{1}{\beta}}(\theta-t)} e^{\rho \theta} \psi(\theta)(\theta-t)^{\beta-1} d \theta .
\end{aligned}
$$

Teorema 4.3. Suponhamos que $u \in X_{0}^{\frac{1}{2}}$ e $u^{\varepsilon} \rightarrow u$ em $X_{\varepsilon}^{\frac{1}{2}}$. Então existe uma função $\bar{v}:\left(0, \varepsilon_{0}\right] \rightarrow$ $\mathbb{R}^{+}, \operatorname{com} \bar{v}(\varepsilon) \stackrel{\varepsilon \rightarrow 0}{\longrightarrow} 0$ que satisfaz

$$
\left\|T_{\varepsilon}\left(t, u^{\varepsilon}\right)-T_{0}(t, u)\right\|_{X_{\varepsilon}^{\frac{1}{2}}} \leqslant \bar{v}(\varepsilon), t \in(0, \tau) .
$$

Demonstração. Os semigrupos não-lineares $T_{\mathcal{E}}\left(t, u^{\varepsilon}\right)$ são dados pela Fórmula da Variação das Constantes,

$$
T_{\mathcal{\varepsilon}}\left(t, u^{\varepsilon}\right)=e^{-A_{\varepsilon} t} u^{\varepsilon}+\int_{0}^{t} e^{-A_{\varepsilon}(t-r)} h\left(T_{\mathcal{\varepsilon}}\left(r, u^{\varepsilon}\right)\right) d r, \quad \varepsilon \in\left[0, \varepsilon_{0}\right] .
$$

Deste modo,

$$
\begin{aligned}
\left\|T_{\mathcal{\varepsilon}}\left(t, u^{\varepsilon}\right)-T_{0}(t, u)\right\|_{X_{\varepsilon}^{\frac{1}{2}}} \leqslant & \left\|e^{-A_{\varepsilon} t} u^{\varepsilon}-e^{-A_{0} t} u\right\|_{X_{\mathcal{\varepsilon}}^{\frac{1}{2}}} \\
& +\int_{0}^{t}\left\|e^{-A_{\varepsilon}(t-r)} h\left(T_{\mathcal{\varepsilon}}\left(r, u^{\varepsilon}\right)\right)-e^{-A_{0}(t-r)} h\left(T_{0}(r, u)\right)\right\|_{X_{\varepsilon}^{\frac{1}{2}}} d r .
\end{aligned}
$$

A partir da continuidade dos semigrupos lineares obtida na seção anterior, obtemos

$$
\begin{aligned}
\left\|e^{-A_{\varepsilon} t} u^{\varepsilon}-e^{-A_{0} t} u\right\|_{X_{\varepsilon}^{\frac{1}{2}}} & \leqslant\left\|e^{-A_{\varepsilon} t} u^{\varepsilon}-e^{-A_{\varepsilon} t} u\right\|_{X_{\varepsilon}^{\frac{1}{2}}}+\left\|e^{-A_{\varepsilon} t} u-e^{-A_{0} t} u\right\|_{X_{\varepsilon}^{\frac{1}{2}}} \\
& \leqslant\left\|e^{-A_{\varepsilon} t}\left(u^{\varepsilon}-u\right)\right\|_{X_{\varepsilon}^{\frac{1}{2}}}+o(1) \\
& \leqslant M_{\omega} e^{-\omega t}\left\|u^{\varepsilon}-u\right\|_{X_{\varepsilon}^{\frac{1}{2}}}+o(1) .
\end{aligned}
$$

De maneira natural podemos estender $h\left(T_{0}(t, u)\right): X_{0}^{\frac{s}{2}} \rightarrow \mathbb{R}$ a $h\left(T_{0}(t, u)\right): X_{\mathcal{\varepsilon}}^{\frac{s}{2}} \rightarrow \mathbb{R}$; ou seja, podemos escrever $h\left(T_{0}(t, u)\right) \in X_{\mathcal{\varepsilon}}^{-\frac{s}{2}}$. Temos então que

$$
\begin{aligned}
\int_{0}^{t} \| e^{-A_{\varepsilon}(t-r)} h\left(T_{\mathcal{\varepsilon}}\left(r, u^{\varepsilon}\right)\right)-e^{-A_{0}(t-r)} & h\left(T_{0}(r, u)\right) \|_{X_{\varepsilon}^{\frac{1}{2}}} d r \leqslant \\
\leqslant & \int_{0}^{t}\left\|e^{-A_{\varepsilon}(t-r)} h\left(T_{\mathcal{\varepsilon}}\left(r, u^{\varepsilon}\right)\right)-e^{-A_{\mathcal{\varepsilon}}(t-r)} h\left(T_{0}(r, u)\right)\right\|_{X_{\varepsilon}^{\frac{1}{2}}} d r \\
& +\int_{0}^{t}\left\|e^{-A_{\varepsilon}(t-r)} h\left(T_{0}(r, u)\right)-e^{-A_{0}(t-r)} h\left(T_{0}(r, u)\right)\right\|_{X_{\varepsilon}^{\frac{1}{2}}} d r .
\end{aligned}
$$


Para $r \in[0, t]$, temos que $h\left(T_{0}(r, u)\right)$ está em um subconjunto compacto de $X_{0}^{-\frac{s}{2}}$, de modo que podemos afirmar que a integral $\int_{0}^{t}\left\|\left[e^{-A_{\varepsilon}(t-r)}-e^{-A_{0}(t-r)}\right] h\left(T_{0}(r, u)\right)\right\|_{X_{\varepsilon}^{\frac{1}{2}}} d r$ é $o(1)$ quando $\varepsilon \rightarrow 0$.

Além disso, se $L_{h}$ é a constante de Lipschitz de $h$, então

$$
\begin{aligned}
\int_{0}^{t}\left\|e^{-A_{\varepsilon}(t-r)}\left[h\left(T_{\mathcal{E}}\left(r, u^{\varepsilon}\right)\right)-h\left(T_{0}(r, u)\right)\right]\right\|_{X_{\varepsilon}^{\frac{1}{2}}} d r \leqslant & \\
& \left.\leqslant \int_{0}^{t} M_{\omega}(t-r)^{-\left(\frac{1+s}{2}\right)} e^{-\omega(t-r)} L_{h} \| T_{\mathcal{E}}\left(r, u^{\varepsilon}\right)-T_{0}(r, u)\right) \|_{X_{\varepsilon}^{\frac{1}{2}}} d r \\
& \left.=M_{\omega} L_{h} \int_{0}^{t}(t-r)^{-\left(\frac{1+s}{2}\right)} e^{-\omega(t-r)} \| T_{\mathcal{E}}\left(r, u^{\varepsilon}\right)-T_{0}(r, u)\right) \|_{X_{\varepsilon}^{\frac{1}{2}}} d r .
\end{aligned}
$$

Logo,

$$
\begin{aligned}
e^{\omega t}\left\|T_{\varepsilon}\left(t, u^{\varepsilon}\right)-T_{0}(t, u)\right\|_{X_{\varepsilon}^{\frac{1}{2}} \leqslant} & M_{\omega}\left\|u^{\varepsilon}-u\right\|_{X_{\varepsilon}^{\frac{1}{2}}}+o(1) e^{\omega t} \\
& +M_{\omega} L_{h} \int_{0}^{t}(t-r)^{-\left(\frac{1+s}{2}\right)} e^{\omega r}\left\|T_{\varepsilon}\left(r, u^{\varepsilon}\right)-T_{0}(r, u)\right\|_{X_{\varepsilon}^{\frac{1}{2}}} d r .
\end{aligned}
$$

Observemos agora que os dois primeiros termos do lado direito da desigualdade acima podem ser majorados por $\tilde{M} v(\varepsilon), \tilde{M}=\tilde{M}(\tau)$, quando $t \in(0, \tau)$ e $v(\varepsilon) \stackrel{\varepsilon \rightarrow 0}{\longrightarrow} 0$. Deste modo, se $\varphi(t)=e^{\omega t}\left\|T_{\varepsilon}\left(t, u^{\varepsilon}\right)-T_{0}(t, u)\right\|_{X_{\varepsilon}^{\frac{1}{2}}}$, temos

$$
\varphi(t) \leqslant \tilde{M} v(\varepsilon)+M_{\omega} L_{h} \int_{0}^{t}(t-r)^{-\left(\frac{1+s}{2}\right)} \varphi(r) d r \quad \text { em }(0, \tau), \tau<\infty
$$

Do Lema 4.2 segue então que

$$
\varphi(t) \leqslant 2 \tilde{M} v(\varepsilon) C
$$

com $C=C\left(M_{\omega}, L, \tau\right)$ constante.

Portanto, existe uma função $\bar{v}:\left(0, \varepsilon_{0}\right] \rightarrow \mathbb{R}^{+}, \operatorname{com} \bar{v}(\varepsilon) \stackrel{\varepsilon \rightarrow 0}{\longrightarrow} 0$ satisfazendo

$$
\left\|T_{\varepsilon}\left(t, u^{\varepsilon}\right)-T_{0}(t, u)\right\|_{X_{\varepsilon}^{\frac{1}{2}}} \leqslant \bar{v}(\varepsilon), \quad t \in(0, \tau)
$$

\subsection{Semicontinuidade superior dos atratores}

Estamos agora aptos a provar o seguinte resultado:

Teorema 4.4. A família de atratores $\left\{\mathscr{A}_{\varepsilon}: \varepsilon \in\left[0, \varepsilon_{0}\right]\right\}$ é semicontínua superiormente em $\varepsilon=0$. 
Demonstração. Consideremos seqüências $\left\{u_{n}\right\}, u_{n} \in \mathscr{A}_{\varepsilon_{n}}, n \in \mathbb{N}$, e $\varepsilon_{n} \rightarrow 0$. Como, do Teorema 4.1. $\sup _{\varepsilon \in\left[0, \varepsilon_{0}\right] w \in \mathscr{A}_{\varepsilon}}\|w\|_{X_{\varepsilon}^{\frac{1}{2}}}<\infty, \overline{\bigcup_{\varepsilon \in\left[0, \varepsilon_{0}\right]} \mathscr{A}_{\varepsilon}}$ é compacto em $X$, existe $u \in X$ tal que $u_{n} \rightarrow u$ fortemente em $X$ e $X_{\varepsilon}^{\frac{1}{2}}$-fracamente. Também temos que

$$
\int_{\Omega} p_{\varepsilon_{n}}(x)\left|\nabla u_{n}^{2}\right| d x \leqslant C_{1} \text {, para alguma constante } C_{1}>0 \text {. }
$$

Logo, procedendo como no Teorema 3.1, concluímos que $u \in X_{0}^{\frac{1}{2}}$. Resta-nos mostrar que $u \in$ $\mathscr{A}_{0}$. Para tanto, é suficiente provarmos que existe uma órbita completa limitada por $u$. Para cada $n \in \mathbb{N}$, temos uma órbita completa limitada

$$
\begin{aligned}
\varphi_{n} & : \mathbb{R} \rightarrow X_{\varepsilon_{n}}^{\frac{1}{2}} \\
t & \mapsto \varphi_{n}\left(t, u_{n}\right)
\end{aligned}
$$

por $u_{n}$. Para $t \geqslant 0$, segue da continuidade dos semigrupos não-lineares que

$$
\varphi_{n}\left(t, u_{n}\right)=T_{\varepsilon_{n}}\left(t, u_{n}\right) \rightarrow T_{0}(t, u) .
$$

Para $t<0$, construímos a órbita por $u$ do seguinte modo: para $t \in(-k,-k+1], k \in \mathbb{Z}^{+}$, consideramos a seqüência $\left\{\varphi_{n}\left(-k, u_{n}\right)\right\}_{n \in \mathbb{N}}$ em $\overline{\bigcup_{\varepsilon \in\left(0, \varepsilon_{0}\right]} \mathscr{A}_{\varepsilon}}$. Seguindo o raciocínio que anteriormente foi usado para a seqüência $\left\{u_{n}\right\}$, obtemos que $\varphi_{n}\left(-k, u_{n}\right) \rightarrow \psi_{0}(-k, u)$. Portanto,

$$
\varphi_{n}\left(t, u_{n}\right)=\varphi_{n}\left(t+k, \varphi_{n}\left(-k, u_{n}\right)\right)=T_{\varepsilon_{n}}\left(t+k, \varphi_{n}\left(-k, u_{n}\right)\right) \rightarrow T_{0}\left(t+k, \varphi_{0}(-k, u)\right):=\tilde{\varphi}_{0}(t, u) .
$$

Finalmente, definindo

$$
\varphi_{0}(t, u)= \begin{cases}T_{0}(t, u), & \text { para } t \geqslant 0 \\ \tilde{\varphi}_{0}(t, u), & \text { para } t<0\end{cases}
$$

segue que $\varphi_{0}(t, u)$ é uma órbita completa limitada por $u$. Portanto, $u \in \mathscr{A}_{0}$.

\subsection{Continuidade dos conjuntos de equilíbrio}

As soluções de equilíbrio de (2.6) e (2.8) são aquelas que independem do tempo, ou seja, as soluções de equilíbrio de $(2.6), \varepsilon \in\left(0, \varepsilon_{0}\right]$, são as soluções da equação elíptica

$$
A_{\varepsilon} u-h(u)=0
$$


e as soluções de

$$
A_{0} u-h(u)=0
$$

são as soluções de equilíbrio do problema limite (2.8).

Definimos $\mathscr{E}_{\varepsilon}=\left\{u: A_{\varepsilon} u-h(u)=0\right\}$ e denotamos a variedade instável de $u_{*}^{\varepsilon} \in \mathscr{E}_{\varepsilon}$ por $\mathrm{W}^{u}\left(u_{*}^{\varepsilon}\right)$, ou seja,

$$
\mathrm{W}^{u}\left(u_{*}^{\varepsilon}\right)=\left\{\eta \in X_{\varepsilon}^{\frac{1}{2}}: u(t, \eta) \text { está definido para todo } t \leqslant 0 \text { e } u(t, \eta) \rightarrow u_{*}^{\varepsilon} \text { quando } t \rightarrow-\infty\right\},
$$

para $\varepsilon \in\left[0, \varepsilon_{0}\right]$.

Antes de darmos mais detalhes de sobre como construir $\mathrm{W}^{u}\left(u_{*}\right)$ ao menos em uma vizinhança de $u_{*}$, vamos olhar por um momento para um problema linear. Considerando a mudança de varáveis $v=u-u_{0}^{*}$ no problema (2.8), somando e subtraindo $h^{\prime}\left(u_{*}\right) v$ ao lado direito, obtemos

$$
\left\{\begin{array}{l}
\dot{v}+\bar{A}_{0} v=h\left(v+u_{*}\right)-h\left(u_{*}\right)-h^{\prime}\left(u_{*}\right) v \\
v(0)=u_{0}-u_{*}=v_{0} .
\end{array}\right.
$$

onde $\bar{A}_{0}=\left(A_{0}-h^{\prime}\left(u_{*}\right)\right)$. Nesta equação, para $v$ muito pequeno, a parte não-linear é muito pequena. É natural então considerarmos o que acontece quando desprezamos a não-linearidade, ou seja, o que acontece com a equação

$$
\left\{\begin{array}{l}
\dot{v}+\bar{A}_{0} v=0 \\
v(0)=v_{0}
\end{array}\right.
$$

Se $Q_{0}^{+}$é a projeção definida pelo espectro de $\bar{A}_{0}$ do lado direito do eixo imaginário, segue que para $v_{0} \in Q_{0} X_{0}^{\frac{1}{2}}$, a solução $v\left(t, v_{0}\right)$ de (4.4) existe para todo $t \leqslant 0$ e $v\left(t, v_{0}\right) \rightarrow 0$ quando $t \rightarrow-\infty$ e $v+u_{*} \rightarrow u_{*}$ quando $t \rightarrow-\infty$. Este fato serve como inspiração para o que queremos fazer. Quando perturbamos (4.4) com uma não-linearidade muito pequena, devemos observar soluções de (4.3) que existem para todo $t \leqslant 0$. Naturalmente, o dado inicial para o qual tais soluções existem não estará em $Q_{0}^{+} X_{0}^{\frac{1}{2}}$, mas em uma variedade não-linear próxima.

\subsubsection{Linearização em torno de um ponto de equilíbrio}

Reservamos esta seção para os resultados concernentes à existência e à convergência das soluções de $\left(\mathrm{E}_{\varepsilon}\right)$ e $\left(\mathrm{E}_{0}\right)$.

Seja $u_{*}$ uma solução de $\left(\mathrm{E}_{\varepsilon}\right)$, e consideremos a mudança de variáveis $v=u-u_{*}$ no problema (2.8). Então, ao adicionarmos e subtrairmos o termo $h^{\prime}\left(u_{*}\right) v$, obtemos

$$
\left\{\begin{array}{l}
\dot{v}+\bar{A}_{0} v=h\left(v+u_{*}\right)-h\left(u_{*}\right)-h^{\prime}\left(u_{*}\right) v \\
v(0)=u-u_{*}=v_{0}
\end{array}\right.
$$


onde $\bar{A}_{0}:=A_{0}-h^{\prime}\left(u_{*}\right)$.

Dizemos que uma solução $u_{*}$ de $\left(\mathrm{E}_{0}\right)$ é hiperbólica se $\sigma\left(A_{0}-h^{\prime}\left(u_{*}\right)\right)$ é disjunto do eixo imaginário.

Assumiremos daqui em diante a seguinte condição de hiperbolicidade:

(H) $\sigma\left(A_{0}-h^{\prime}\left(u_{*}\right)\right) \cap\{\mu \in \mathbb{C}: \operatorname{Re} \mu=0\}=\varnothing$.

Teorema 4.5 (Teorema de Schauder). Sejam E um espaço vetorial normado e $B \subset$ E compacto e convexo. Então toda função contínua $T: B \rightarrow B$ possui um ponto fixo.

A proposição seguinte garante que $\mathscr{E}_{0}=\left\{u: A_{0} u-h(u)=0\right\}$ tem cardinalidade finita.

Proposição 4.6. $S e \mathscr{E}_{0}=\left\{u: A_{0} u-h(u)=0\right\}$ tem apenas pontos isolados, então $\mathscr{E}_{0}$ tem cardinalidade finita. Todo ponto de equilíbrio hiperbólico $u_{*}$ de (2.7) é isolado.

Demonstração. Observemos primeiramente que a não-linearidade $h: X_{0}^{\frac{1}{2}} \rightarrow X_{0}^{-\frac{s}{2}}$ é uniformemente limitada:

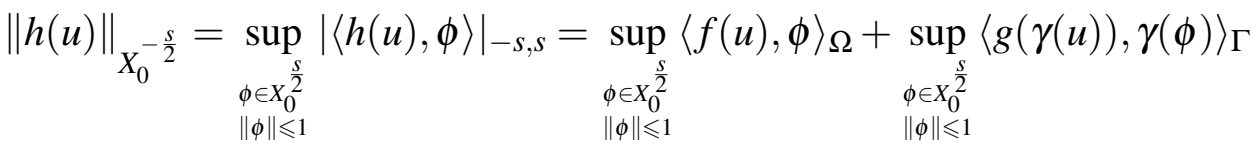

$$
\begin{aligned}
& \leqslant \sup _{\substack{\phi \in X_{0}^{\frac{s}{2}} \\
\|\phi\| \leqslant 1}} \int_{\Omega} f(u(x)) \phi(x) d x+\sup _{\substack{\phi \in X_{0}^{\frac{s}{2}} \\
\|\phi\| \leqslant 1}} \int_{\Gamma} g(\gamma(u(x))) \gamma(\phi(x)) d x \\
& \leqslant \sup _{\substack{\phi \in X_{0}^{2} \\
\|\phi\| \leqslant 1}}\left(\int_{\Omega} f(u(x))^{2} d x\right)^{\frac{1}{2}}\left(\int_{\Omega} \phi(x)^{2} d x\right)^{\frac{1}{2}} \\
& +\sup _{\substack{\frac{s}{2} \\
\phi \in X_{0}}}\left(\int_{\Gamma} g(\gamma(u(x)))^{2} d x\right)^{\frac{1}{2}}\left(\int_{\Gamma} \gamma(\phi(x))^{2} d x\right)^{\frac{1}{2}} \\
& \|\phi\| \leqslant 1 \\
& \leqslant C_{f} \sup _{s}\|\phi\|_{L^{2}(\Omega)}+C_{g} \sup _{s}\|\gamma(\phi)\|_{L^{2}(\Gamma)} \\
& \begin{array}{ll}
\phi \in X_{0}^{\frac{s}{2}} & \phi \in X_{0}^{\frac{s}{2}} \\
\|\phi\| \leqslant 1 & \|\phi\| \leqslant 1
\end{array} \\
& \leqslant C_{1} C_{f} \sup _{\substack{\phi \in X_{0}^{\frac{s}{2}} \\
\|\phi\| \leqslant 1}}\|\phi\|_{X_{0}^{\frac{s}{2}}}+C_{2} C_{g} \sup _{\substack{\phi \in X_{0}^{\frac{s}{2}} \\
\|\phi\| \leqslant 1}}\|\phi\|_{X_{0}^{\frac{s}{2}}} \\
& \leqslant C_{h},
\end{aligned}
$$

onde $C_{1}$ e $C_{2}$ são constantes de imersão e $C_{h}=C_{1} C_{f}+C_{2} C_{g}$. Logo, a aplicação

$$
X_{0}^{\frac{1}{2}} \ni u \mapsto A_{0}^{-1} h(u) \in X_{0}^{1-\frac{s}{2}} \subset \subset X_{0}^{\frac{1}{2}}
$$


é compacta de $X_{0}^{\frac{1}{2}}$ em $X_{0}^{\frac{1}{2}}$ e, além disso, se $C_{3}$ é a constante de imersão de $X_{0}^{1-\frac{s}{2}}$ em $X_{0}^{\frac{1}{2}}$, então esta aplicação leva $X_{0}^{\frac{1}{2}}$ na bola $B_{X_{0}^{\frac{1}{2}}}\left(0, C_{3} C_{h}\right)$, pois

$$
\begin{aligned}
\left\|A_{0}^{-1} h(u)\right\|_{X_{0}^{\frac{1}{2}}} & \leqslant C_{3}\left\|A_{0}^{-1} h(u)\right\|_{X^{1-\frac{s}{2}}}=C_{3}\left\|A_{0}^{1-\frac{s}{2}} A_{0}^{-1} h(u)\right\|_{X_{0}} \\
& =C_{3}\left\|A_{0}^{-\frac{s}{2}} h(u)\right\|_{X_{0}}=C_{3}\|h(u)\|_{X_{0}^{-\frac{s}{2}}} \leqslant C_{3} C_{h} .
\end{aligned}
$$

Segue portanto do Teorema de Schauder que $\left(\mathrm{E}_{0}\right)$ tem ao menos uma solução.

Já que $A_{0}$ tem resolvente compacto, segue que o conjunto $\mathscr{E}_{0}=\left\{u: A_{0} u-h(u)=0\right\}$ é compacto em $X_{0}^{\frac{1}{2}}$. Se mostrarmos que toda solução de $\left(\mathrm{E}_{0}\right)$ é isolada, então estará provado que existe apenas um número finito delas. Para tanto, notemos que $u \in \mathscr{E}_{0}$ é uma solução de $\left(\overline{\mathrm{E}_{0}}\right)$ se, e somente se, $u$ é ponto fixo de

$$
\Phi(u)=-\left(A_{0}-h^{\prime}\left(u_{*}\right)\right)^{-1}\left(h^{\prime}\left(u_{*}\right) u-h(u)\right) .
$$

Vamos mostrar que existe $\delta>0$ tal que $\Phi: \overline{B_{X_{0}^{\frac{1}{2}}}\left(u_{*}, \delta\right)} \rightarrow \overline{B_{X_{0}^{\frac{1}{2}}}\left(u_{*}, \delta\right)}$ é uma contração. Neste caso, $u_{*}$ será o único elemento de $\mathscr{E}_{0}$ em $B=\overline{B_{X_{0}^{\frac{1}{2}}}\left(u_{*}, \delta\right)}$ e, portanto, isolado. Para ver isto, observemos que se $u, v \in B$, então

$$
\begin{aligned}
\|\Phi(u)-\Phi(v)\|_{X_{0}^{\frac{1}{2}}} & =\left\|\left(A_{0}-h^{\prime}\left(u_{*}\right)\right)^{-1}\left[h(u)-h(v)-h^{\prime}\left(u_{*}\right)(u-v)\right]\right\|_{X_{0}^{\frac{1}{2}}} \\
& =\left\|A_{0}^{-\frac{s}{2}} A_{0}^{\frac{s}{2}} A_{0}^{\frac{1}{2}}\left(A_{0}-h^{\prime}\left(u_{*}\right)\right)^{-1}\left[h(u)-h(v)-h^{\prime}\left(u_{*}\right)(u-v)\right]\right\|_{X_{0}} \\
& =\left\|A_{0}^{\frac{1+s}{2}}\left(A_{0}-h^{\prime}\left(u_{*}\right)\right)^{-1}\left[h(u)-h(v)-h^{\prime}\left(u_{*}\right)(u-v)\right]\right\|_{X_{0}^{-\frac{s}{2}}} \\
& =\left\|A_{0}^{-\left(\frac{1-s}{2}\right)} A_{0}\left(A_{0}-h^{\prime}\left(u_{*}\right)\right)^{-1}\left[h(u)-h(v)-h^{\prime}\left(u_{*}\right)(u-v)\right]\right\|_{X_{0}^{-\frac{s}{2}}} \\
& \leqslant C_{4}\left\|h(u)-h(v)-h^{\prime}\left(u_{*}\right)(u-v)\right\|_{X_{0}^{-\frac{s}{2}}} \\
& \leqslant C_{4}\left\|\left[h^{\prime}(\theta u-(1-\theta) v)-h^{\prime}\left(u_{*}\right)\right](u-v)\right\|_{X_{0}^{\frac{-s}{2}}} \\
& \leqslant C_{5}\left\|(\theta u-(1-\theta) v)-u_{*}\right\|_{X_{0}^{\frac{1}{2}}\|u-v\|_{X_{0}^{\frac{1}{2}}}} \\
& \leqslant 2 C_{5} \delta\|u-v\|_{X_{0}^{\frac{1}{2}} \cdot}
\end{aligned}
$$

Assim, escolhendo $\delta$ tal que $2 C_{5} \delta<\frac{1}{2}$, obtemos que $\Phi$ é uma contração. Notemos ainda que se $v \in B$, então $\left\|\Phi(v)-u_{*}\right\|_{X_{0}^{\frac{1}{2}}}=\left\|\Phi(v)-\Phi\left(u_{*}\right)\right\|_{X_{0}^{\frac{1}{2}}} \leqslant\left\|v-u_{*}\right\|_{X_{0}^{\frac{1}{2}}}<\delta$, o que mostra que $\Phi(B) \subset B$, ou seja, $\Phi$ tem um único ponto fixo em $B$ e o resultado está demonstrado. 
A semicontinuidade superior da família dos conjuntos de equilíbrio $\left\{\mathscr{E}_{\varepsilon}: \varepsilon \in\left(0, \varepsilon_{0}\right]\right\}$ é uma conseqüência imediata da convergência compacta:

Proposição 4.7. Suponhamos que exista uma seqüência $\varepsilon_{n} \rightarrow 0^{+}$tal que $\left(\mathrm{E}_{\varepsilon}\right)$ tenha uma solução $u_{\varepsilon_{n}}$, para cada $n \in \mathbb{N}$. Então, tomando subseqüências se necessário, existe uma solução $u_{*}$ de $\left(\underline{\mathrm{E}_{0}}\right)$ de forma que $\left\|u_{\varepsilon_{n}}-u_{*}\right\|_{X_{\varepsilon}^{\frac{1}{2}}} \rightarrow 0$ quando $\varepsilon \rightarrow 0$.

Demonstração. Como $0 \in \rho\left(A_{\varepsilon_{n}}\right)$, temos

$$
u_{\varepsilon_{n}}=A_{\varepsilon_{n}}^{-1} h\left(u_{\varepsilon_{n}}\right)
$$

Temos que a família $\left\{A_{\varepsilon_{n}}^{-1}: n \in \mathbb{N}\right\}$ é coletivamente compacta e também que $\left|h\left(u_{\varepsilon_{n}}\right)\right| \leqslant C_{h}$, para todo $n \in N$, e assim existem uma subseqüência que denotaremos também por $u_{\varepsilon_{n}}$, e $u_{*}$ tais que $u_{\varepsilon_{n}} \rightarrow u_{*}$ em $X_{\varepsilon}^{\frac{1}{2}}$.

Da continuidade do operador resolvente e da continuidade da função $h$ segue que $u_{*}=$ $A_{0}{ }^{-1} h\left(u_{*}\right)$, de modo que $u_{*}$ é solução de $\left(\mathrm{E}_{0}\right)$.

Uma medida de não-compacidade $\beta$ em um espaço métrico completo $Y$ é uma função $\beta$ : $2^{Y} \rightarrow \mathbb{R}^{+}$que satisfaz as seguintes propriedades:

(i) $\beta(A)=0$ se, e somente se, $A$ é pré-compacto.

(ii) $\beta(A \cup B)=\max \{\beta(A), \beta(B)\}$.

(iii) $\beta(A+B) \leqslant \beta(A)+\beta(B)$.

Lema 4.8. Para todo $0<\theta<1$, a seqüência $\left\{A_{\varepsilon}^{-\theta}\right\}$ é coletivamente compacta e $A_{\varepsilon}^{-\theta} \stackrel{\varepsilon \rightarrow 0}{\longrightarrow} A_{0}^{-\theta}$ em $X$.

Demonstração. Sejam $\Sigma_{-\lambda, \phi}$ como na condição de estabilidade (E) e $\Gamma$ a fronteira de $\Sigma_{-\lambda, \phi}$. Como

$$
A_{\varepsilon}^{-\theta}=\frac{1}{2 \pi i} \int_{\Gamma} \mu^{-\theta}\left(\mu+A_{\varepsilon}\right)^{-1} d \mu
$$

segue que a integral acima é absolutamente uniformemente convergente para $\varepsilon \in\left(0, \varepsilon_{\lambda}\right]$. Logo, dado $\eta>0$, podemos dividir o contorno $\Gamma=\Gamma_{1}^{\eta} \cup \Gamma_{2}^{\eta}$ de tal maneira que $\Gamma_{1}^{\eta}$ seja limitada e a integral

$$
\frac{1}{2 \pi i} \int_{\Gamma_{2}^{\eta}}\left\|\mu^{-\theta}\left(\mu+A_{\varepsilon}\right)^{-1}\right\|_{\mathscr{L}(X)} d \mu \leqslant \eta, \quad \text { para todo } \varepsilon \in\left(0, \varepsilon_{\lambda}\right]
$$


Sobre $\Gamma_{1}^{\eta}$, reescrevemos a integral como

$$
B_{\varepsilon}:=\frac{A_{\varepsilon}^{-1}}{2 \pi i} \int_{\Gamma_{1}^{\eta}} \mu^{-\theta} A_{\varepsilon}\left(\mu+A_{\varepsilon}\right)^{-1} d \mu
$$

e usamos que $\left\|\mu^{-\theta} A_{\varepsilon}\left(\mu+A_{\varepsilon}\right)^{-1}\right\|_{X}=\left\|\mu^{-\theta}\left(I+\mu A_{\varepsilon}{ }^{-1}\right)^{-1}\right\|_{X}$ é uniformemente limitado para $\varepsilon \in\left(0, \varepsilon_{\lambda}\right]$ e também que $\left\{A_{\varepsilon}^{-1}: \varepsilon \in\left(0, \varepsilon_{\lambda}\right]\right\}$ é coletivamente compacto para garantir que $\left\{B_{\varepsilon}\right.$ : $\left.\varepsilon \in\left(0, \varepsilon_{\lambda}\right]\right\}$ é coletivamente compacto. Seja $\beta: 2^{X} \rightarrow \mathbb{R}^{+}$uma medida de não-compacidade. Agora, tomando quaisquer seqüências $\varepsilon_{n} \rightarrow 0$ e $\left\{u_{n}\right\}, u_{n} \in X,\left\|u_{n}\right\|_{X}=1$, obtemos

$$
\beta\left(\left\{A_{\varepsilon_{n}}^{-\theta} u_{n}\right\}\right) \leqslant \beta\left(B_{\varepsilon_{n}}\left\{u_{n}\right\}\right)+\beta\left(\frac{1}{2 \pi i} \int_{\Gamma_{2}^{\eta}} \mu^{-\theta}\left(\mu+A_{\varepsilon_{n}}\right)^{-1} d \mu\left\{u_{n}\right\}\right) \leqslant \eta .
$$

Logo, dadas seqüências $\varepsilon_{n} \rightarrow 0$ e $\left\{u_{n}\right\}, \operatorname{com} u_{n} \in X,\left\|u_{n}\right\|_{X}=1$, temos que $\beta\left(\left\{A_{\varepsilon_{n}}^{-\theta} u_{n}\right\}\right)=0$ e que $\left\{A_{\varepsilon}^{-\theta}: \varepsilon \in\left(0, \varepsilon_{\lambda}\right]\right\}$ é coletivamente compacta.

Do Teorema da Convergência Dominada e do Lema 3.5, temos

$$
A_{\varepsilon}^{-\theta}=\frac{1}{2 \pi i} \int_{\Gamma}\left\|-\mu^{-\theta}\left(\mu+A_{\varepsilon}\right)^{-1}\right\|_{\mathscr{L}(X)} d \mu \stackrel{\varepsilon \rightarrow 0}{\longrightarrow} \frac{1}{2 \pi i} \int_{\Gamma}\left\|\mu^{-\theta}\left(\mu+A_{0}\right)^{-1}\right\|_{\mathscr{L}(X)} d \mu=A_{0}^{-\theta}
$$

para todo $\mu \in \Gamma$.

Pretendemos agora mostrar a convergência compacta $\overline{A_{\mathcal{E}}} \rightarrow \overline{A_{0}}$. Para tanto, devemos estudar a família de operadores $\left\{h^{\prime}\left(u_{*}^{\varepsilon}\right): \varepsilon \in\left(0, \varepsilon_{0}\right]\right\}$. Prosseguimos então com o seguinte resultado:

Lema 4.9. Seja $\left\{u^{\varepsilon}\right\}$ uma seqüência tal que $u^{\varepsilon} \rightarrow u \in X_{0}^{\frac{1}{2}}$ em $X_{\varepsilon}^{\frac{1}{2}}$, e consideremos a família de operadores

$$
\begin{aligned}
D_{\varepsilon}: X_{\mathcal{\varepsilon}}^{\frac{1}{2}} & \rightarrow X_{\varepsilon}^{-\frac{s}{2}} \\
v & \mapsto D_{\varepsilon} v=h^{\prime}\left(u^{\varepsilon}\right) v=f_{\Omega}^{\prime}\left(u^{\varepsilon}\right) v+g_{\Omega}^{\prime}\left(u^{\varepsilon}\right) v
\end{aligned}
$$

e também o operador

$$
\begin{aligned}
D_{0}: X_{0}^{\frac{1}{2}} & \rightarrow X_{0}^{-\frac{s}{2}} \\
v & \mapsto D_{0} v=h^{\prime}(u) v=f_{\Omega}^{\prime}(u) v+g_{\Gamma}^{\prime}(u) v .
\end{aligned}
$$

Então, se $\left\{z_{\varepsilon}\right\}$ é uma seqüência tal que $z_{\varepsilon} \rightarrow z \in X_{0}^{\frac{1}{2}}$ em $X_{\mathcal{\varepsilon}}^{\frac{1}{2}}$, temos que $D_{\varepsilon} z_{\varepsilon} \rightarrow D_{0} z$ em $X_{\varepsilon}^{-\frac{s}{2}}$ quando $\varepsilon \rightarrow 0$. 


\section{Demonstração.}

$$
\begin{aligned}
& \left\|h^{\prime}\left(u^{\varepsilon}\right) z-h^{\prime}(u) z\right\|_{X_{\varepsilon}^{-\frac{s}{2}}} \leqslant\left\|f_{\Omega}^{\prime}\left(u^{\varepsilon}\right) z-f_{\Omega}^{\prime}(u) z\right\|_{X_{\varepsilon}^{-\frac{s}{2}}}+\left\|g_{\Gamma}^{\prime}\left(\gamma\left(u^{\varepsilon}\right)\right) \gamma(z)-g_{\Gamma}^{\prime}(\gamma(u)) \gamma(z)\right\|_{X_{\varepsilon}^{-\frac{s}{2}}} \\
& \leqslant \sup _{\substack{\phi \in X_{0}^{2} \\
\|\phi\| \leqslant 1}}\left|\left\langle f^{\prime}\left(u^{\varepsilon}\right) z-f^{\prime}(u) z, \phi\right\rangle_{\Omega}\right| \\
& +\sup _{\substack{\phi \in X_{0}^{2} \\
\|\phi\| \leqslant 1}} \mid\left\langle g^{\prime}\left(\gamma\left(u^{\varepsilon}\right) \gamma(z)-g^{\prime}(\gamma(u)) \gamma(z), \gamma(\phi)\right\rangle_{\Gamma}\right| \\
& \leqslant \sup _{\substack{\phi \in X_{0}^{2} \\
\|\phi\| \leqslant 1}} \int_{\Omega}\left|\left[f^{\prime}\left(u^{\varepsilon}(x)\right)-f^{\prime}(u(x))\right] z(x) \phi(x)\right| d x \\
& +\sup _{\substack{\phi \in X_{0}^{2} \\
\|\phi\| \leqslant 1}} \int_{\Gamma}\left|\left[g^{\prime}\left(\gamma\left(u^{\varepsilon}(x)\right)\right)-g^{\prime}(\gamma(u(x)))\right] \gamma(z(x)) \gamma(\phi(x))\right| d x \\
& \leqslant \sup _{\substack{\phi \in X_{0}^{2} \\
\|\phi\| \leqslant 1}}\left(\int_{\Omega}\left|f^{\prime}\left(u^{\varepsilon}(x)\right) z(x)-f^{\prime}(u(x)) z(x)\right|^{2} d x\right)^{\frac{1}{2}}\left(\int_{\Omega}|\phi(x)|^{2} d x\right)^{\frac{1}{2}} \\
& +\sup _{\substack{1 \\
\phi \in X_{0}^{2} \\
\|\phi\| \leqslant 1}}\left(\int_{\Gamma}\left|\left[g^{\prime}\left(\gamma\left(u^{\varepsilon}(x)\right)\right) \gamma(z(x))-g^{\prime}(\gamma(u(x)))\right] \gamma(z(x))\right|^{2} d x\right)^{\frac{1}{2}} \\
& \left(\int_{\Gamma}|\gamma(\phi(x))|^{2} d x\right)^{\frac{1}{2}} \\
& \leqslant \sup _{\substack{\phi \in X_{0}^{\frac{1}{2}} \\
\|\phi\| \leqslant 1}}\left(\int_{\Omega}\left|f^{\prime \prime}\left(\theta(x) u^{\varepsilon}(x)-(1-\theta(x)) u(x)\right)\right|^{2}\left|u^{\varepsilon}(x)-u(x)\right|^{2}|z(x)|^{2} d x\right)^{\frac{1}{2}} \\
& \times\|\phi\|_{L^{2}(\Omega)} \\
& +\sup _{\substack{\phi \in X_{0}^{\frac{1}{2}} \\
\|\phi\| \leqslant 1}}\left(\int_{\Gamma}\left|g^{\prime \prime}\left(\gamma\left(\theta(x) u^{\varepsilon}(x)-(1-\theta(x)) u(x)\right)\right)\right|^{2}\left|\gamma\left(u^{\varepsilon}(x)-u(x)\right)\right|^{2}\right. \\
& \left.|\gamma(z(x))|^{2} d x\right)^{\frac{1}{2}}\|\gamma(\phi)\|_{L^{2}(\Gamma)} \\
& \leqslant C_{1} C_{f} \sup _{\substack{\frac{1}{2} \\
\phi \in X_{0}^{2} \\
\|\phi\| \leqslant 1}}\left(\int_{\Omega}\left|u^{\varepsilon}(x)-u(x)\right|^{2}|z(x)|^{2} d x\right)^{\frac{1}{2}}\|\phi\|_{X_{0}^{\frac{1}{2}}} \\
& +C_{2} C_{g} \sup _{\substack{\phi \in X_{0} \\
\|\phi\| \leqslant 1}}\left(\int_{\Gamma}\left|\gamma\left(u^{\varepsilon}(x)-u(x)\right)\right|^{2}|\gamma(z(x))|^{2} d x\right)^{\frac{1}{2}}\|\gamma(\phi)\|_{H^{s-\frac{1}{2}}(\Gamma)} \text {. }
\end{aligned}
$$


Assim,

$$
\begin{aligned}
\left\|h^{\prime}\left(u^{\varepsilon}\right) z-h^{\prime}(u) z\right\|_{X_{\varepsilon}^{-\frac{s}{2}}} & \leqslant C_{3}\left\|\left(u^{\varepsilon}-u\right)^{2}\right\|_{L^{2}(\Omega)}\left\|z^{2}\right\|_{L^{2}(\Omega)} \\
& +C_{4} \sup _{\substack{\frac{1}{2} \\
\| X_{0}^{2}}}\left\|\left(\gamma\left(u^{\varepsilon}-u\right)\right)^{2}\right\|_{L^{2}(\Gamma)}\left\|(\gamma(z))^{2}\right\|_{L^{2}(\Gamma)}\|\phi\|_{H^{s}(\Omega)} \\
& \leqslant C_{3}\left\|u^{\varepsilon}-u\right\|_{L^{4}(\Omega)}\|z\|_{L^{4}(\Omega)}+C_{5}\left\|\left(\gamma\left(u^{\varepsilon}-u\right)\right)^{2}\right\|_{L^{2}(\Gamma)}\left\|(\gamma(z))^{2}\right\|_{L^{2}(\Gamma)} \\
& \leqslant C_{6}\left\|u^{\varepsilon}-u\right\|_{X_{\varepsilon}^{\frac{1}{2}}}\|z\|_{X_{0}^{\frac{1}{2}}}+C_{7}\left\|\left(\gamma\left(u^{\varepsilon}-u\right)\right)^{2}\right\|_{H^{s-\frac{1}{2}}(\Gamma)}\left\|(\gamma(z))^{2}\right\|_{H^{s-\frac{1}{2}(\Gamma)}} \\
& \leqslant C_{6}\left\|u^{\varepsilon}-u\right\|_{X_{\varepsilon}^{\frac{1}{2}}}\|z\|_{X_{0}^{\frac{1}{2}}}+C_{8}\left\|\left(u^{\varepsilon}-u\right)^{2}\right\|_{X_{\varepsilon}^{\frac{s}{2}}}\left\|z^{2}\right\|_{X_{0}^{\frac{s}{2}}} \\
& \leqslant C_{6}\left\|u^{\varepsilon}-u\right\|_{X_{\varepsilon}^{\frac{1}{2}}}\|z\|_{X_{0}^{\frac{1}{2}}}+C_{9}\left\|u^{\varepsilon}-u\right\|_{X}\|z\|_{X} \\
& \leqslant C_{10}\left\|u^{\varepsilon}-u\right\|_{X_{\varepsilon}^{\frac{1}{2}}}\|z\|_{X_{0}^{\frac{1}{2}}}^{\stackrel{\varepsilon \rightarrow 0}{\longrightarrow} 0 .}
\end{aligned}
$$

Deste modo,

$$
\begin{aligned}
\left\|D_{\varepsilon} z_{\varepsilon}-D_{0} z\right\|_{X_{\varepsilon}^{-\frac{s}{2}}} & \leqslant\left\|h^{\prime}\left(u^{\varepsilon}\right) z_{\varepsilon}-h^{\prime}\left(u^{\varepsilon} 7\right) z\right\|_{X_{\varepsilon}^{-\frac{s}{2}}}+\left\|h^{\prime}\left(u^{\varepsilon}\right) z-h^{\prime}(u) z\right\|_{X_{\varepsilon}^{-\frac{s}{2}}} \\
& \leqslant\left\|h^{\prime}\left(u^{\varepsilon}\right)\right\|_{\mathscr{L}\left(X_{\varepsilon}^{\frac{1}{2}}, X_{\varepsilon}^{-\frac{s}{2}}\right)}\left\|z_{\varepsilon}-z\right\|_{X_{\varepsilon}^{\frac{1}{2}}}+o(1) \stackrel{\varepsilon \rightarrow 0}{\longrightarrow} 0 .
\end{aligned}
$$

Lema 4.10. Para todo $0 \leqslant \theta<1$, existe um $\bar{\varepsilon} \in\left(0, \varepsilon_{0}\right]$ de tal modo que

$$
\left\{\left(A_{\varepsilon}\right)^{\theta}\left(A_{\varepsilon}-D_{\varepsilon}\right)^{-1}: 0<\varepsilon \leqslant \varepsilon_{\mu}\right\}
$$

é coletivamente compacto, uniformemente limitado e $\left(A_{\varepsilon}\right)^{\theta}\left(A_{\varepsilon}-D_{\varepsilon}\right)^{-1} \stackrel{\varepsilon \rightarrow 0}{\longrightarrow}\left(A_{0}\right)^{\theta}\left(A_{0}-D_{0}\right)^{-1}$ compactamente.

Demonstração. Sejam $\alpha \in\left(0, \frac{1}{2}\right)$ fixado, $r_{1}$ e $r_{2}$ tais que $1>\alpha+r_{1} \geqslant \theta$ e $r_{2}=1-\alpha-r_{1}$. Notemos que

$$
\left(A_{\varepsilon}\right)^{\theta}\left(A_{\varepsilon}-D_{\varepsilon}\right)^{-1}=\left(A_{\varepsilon}\right)^{-\left(\alpha+r_{1}-\theta\right)}\left(I-\left(A_{\varepsilon}\right)^{-r_{2}} D_{\varepsilon}\left(A_{\varepsilon}\right)^{-\alpha-r_{1}}\right)^{-1}\left(A_{\varepsilon}\right)^{-r_{2}} .
$$

De fato,

$$
\begin{aligned}
A_{\varepsilon}{ }^{-\left(\alpha+r_{1}-\theta\right)}\left[I-A_{\varepsilon}{ }^{-r_{2}} D_{\varepsilon} A_{\varepsilon}{ }^{-\alpha-r_{1}}\right]^{-1} A_{\varepsilon}{ }^{-r_{2}} & =A_{\varepsilon}{ }^{\theta} A_{\varepsilon}{ }^{-\alpha-r_{1}}\left[A_{\varepsilon}^{r_{2}}\left(I-A_{\varepsilon}{ }^{-r_{2}} D_{\varepsilon} A_{\varepsilon}{ }^{-\alpha-r_{1}}\right)\right]^{-1} \\
& =A_{\varepsilon}{ }^{\theta}\left[A_{\varepsilon}{ }^{r_{2}}\left(I-A_{\varepsilon}{ }^{-r_{2}} D_{\varepsilon} A_{\varepsilon}{ }^{-\alpha-r_{1}}\right) A_{\varepsilon}{ }^{\alpha+r_{1}}\right]^{-1} \\
& =A_{\varepsilon}{ }^{\theta}\left[\left(A_{\varepsilon}{ }^{r_{2}}-D_{\varepsilon} A_{\varepsilon}{ }^{-\alpha-r_{1}}\right) A_{\varepsilon}{ }^{\alpha+r_{1}}\right]^{-1} \\
& =A_{\varepsilon}{ }^{\theta}\left(A_{\varepsilon}-D_{\varepsilon}\right)^{-1}
\end{aligned}
$$


Como $\alpha+r_{1}<1$ e $r_{2}<1$, segue do Lema 4.8 que $\left\{\left(A_{\varepsilon}\right)^{-r_{2}} D_{\varepsilon}\left(A_{\varepsilon}\right)^{-\alpha-r_{1}}\right\}$ é coletivamente compacto. Logo, pelo Lema 3.4, $\left(I-A_{\mathcal{\varepsilon}}{ }^{-r_{2}} D_{\varepsilon} A_{\varepsilon}{ }^{-\alpha-r_{1}}\right)^{-1}$ é uniformemente limitado, pois $\left(A_{\varepsilon}{ }^{-r_{2}} D_{\varepsilon} A_{\varepsilon}{ }^{-\alpha-r_{1}}\right)^{-1} \rightarrow\left(A_{0}^{-r_{2}} D_{0} A_{0}^{-\alpha-r_{1}}\right)$ compactamente. Por fim, sendo $\alpha+r_{1}-\theta<1$, o resultado segue.

Reunimos a seguir os resultados referentes aos operadores $\overline{A_{\varepsilon}}$ e $\overline{A_{0}}$ dos problemas linearizados obtidos anteriormente .

Lema 4.11. Existem um setor $\Sigma_{\omega, \varphi}, \varphi<\frac{\pi}{2}, \omega \in \mathbb{R}^{+}$, uma constante $\bar{\varepsilon}$ e uma constante $\bar{M}$ de tal modo que

$$
\left\|\left(\mu+\bar{A}_{\varepsilon}\right)^{-1}\right\|_{\mathscr{L}(X)} \leqslant \frac{\bar{M}}{|\mu-\omega|},
$$

para todo $\mu \in \Sigma_{\omega, \varphi}$ e para todo $\varepsilon \in(0, \bar{\varepsilon}]$.

Demonstração. Segue da hipótese (E) e da Desigualdade do Momento que, para $\mu \in \Sigma_{0, \phi} \subset$ $\rho\left(-A_{\varepsilon}\right)$,

$$
\left\|A_{\mathcal{\varepsilon}}^{\frac{1}{2}}\left(\mu+A_{\varepsilon}\right)^{-1}\right\|_{\mathscr{L}(X)} \leqslant C_{1} \frac{M^{\frac{1}{2}}}{|\mu|^{\frac{1}{2}}}, \varepsilon \in\left(0, \varepsilon_{0}\right] .
$$

Temos que $D_{\varepsilon}\left(A_{\varepsilon}\right)^{-\frac{1}{2}}$ é uniformemente limitado em $\varepsilon$ e

$$
\left(\mu+A_{\varepsilon}-D_{\varepsilon}\right)=\left[I-D_{\varepsilon}\left(A_{\varepsilon}\right)^{-\frac{1}{2}}\left(A_{\varepsilon}\right)^{\frac{1}{2}}\left(\mu+A_{\varepsilon}\right)^{-1}\right]\left(\mu+A_{\varepsilon}\right) .
$$

Seja $R>0$ de tal maneira que $\left\|D_{\varepsilon}\left(A_{\mathcal{E}}\right)^{-\frac{1}{2}}\left(A_{\mathcal{E}}\right)^{\frac{1}{2}}\left(\mu+A_{\mathcal{\varepsilon}}\right)^{-1}\right\|_{\mathscr{L}(X)}<\frac{1}{2}$ para $|\mu|>R$ e $\mu \in \Sigma_{0, \phi}$. Então o operador $\left(I-D_{\varepsilon}\left(A_{\varepsilon}\right)^{-\frac{1}{2}}\left(A_{\mathcal{\varepsilon}}\right)^{\frac{1}{2}}\left(\mu+A_{\mathcal{\varepsilon}}\right)^{-1}\right)$ é invertível e

$$
\left\|\left[I-D_{\mathcal{\varepsilon}}\left(A_{\mathcal{\varepsilon}}\right)^{-\frac{1}{2}}\left(A_{\mathcal{\varepsilon}}\right)^{\frac{1}{2}}\left(\mu+A_{\mathcal{E}}\right)^{-1}\right]^{-1}\right\|_{\mathscr{L}(X)} \leqslant 2
$$

Logo, se $\omega>R$ e $\varphi$ é escolhido de forma que $\Sigma_{\omega, \varphi} \subset\left\{\mu \in \Sigma_{0, \phi}:|\mu|>R\right\}$, temos

$$
\begin{aligned}
\left\|\left(\mu+\overline{A_{\mathcal{E}}}\right)^{-1}\right\|_{\mathscr{L}(X)} & \leqslant 2\left\|\left(\mu+A_{\mathcal{E}}\right)^{-1}\right\|_{\mathscr{L}(X)} \\
& \leqslant 2 \frac{M_{E}}{|\mu+\lambda|} \leqslant \frac{\bar{M}}{|\mu-\omega|} .
\end{aligned}
$$

Notemos que, do Lema $4.10, \bar{A}_{\varepsilon}^{-1}$ converge compactamente para $\bar{A}_{0}^{-1}$. Assim, procedendo da mesma forma como no Lema 3.7, obtemos o seguinte resultado: 
Lema 4.12. de $\rho\left(-\overline{A_{0}}\right)$. Então existe uma constante $\varepsilon_{K}>0$ de tal forma que $K \subset \rho\left(-\overline{A_{\varepsilon}}\right)$ para todo $\varepsilon \in\left(0, \varepsilon_{K}\right] e$

$$
\sup _{\alpha \in[0,1]} \sup _{\varepsilon \in\left(0, \varepsilon_{K}\right]} \sup _{\mu \in K}\left\|\left(\mu+\overline{A_{\varepsilon}}\right)^{-1}\right\|_{\mathscr{L}\left(X, X_{\varepsilon}^{\alpha}\right)}<\infty .
$$

Se $f_{\Omega} \in L^{2}(\Omega), g_{\Gamma} \in L^{2}(\Gamma)$ e $h=f_{\Omega}+g_{\Gamma} \in X_{0}^{-\frac{s}{2}}$ é definida por

$$
\langle h, \phi\rangle_{-s, s}=\int_{\Omega} f_{\Omega} \phi+\int_{\Gamma} g_{\Gamma} \phi, \quad \forall \phi \in X_{0}^{\frac{s}{2}},
$$

então estendemos $h$ a $X_{\mathcal{\varepsilon}}^{-\frac{s}{2}}$ de maneira natural, e obtemos

$$
\sup _{\mu \in K}\left\|\left(\mu+\overline{A_{\varepsilon}}\right)^{-1} h-\left(\mu+\overline{A_{0}}\right)^{-1} h\right\|_{X_{\varepsilon}^{\frac{1}{2}}} \stackrel{\varepsilon \rightarrow 0}{\longrightarrow} 0
$$

Além disso, se J é um subconjunto de $X_{0}^{-\frac{s}{2}}$ constituído por elementos da forma $f_{\Omega}+g_{\Gamma}$, com $f_{\Omega}$ $\left(g_{\Gamma}\right)$ variando em um subconjunto limitado de $L^{2}(\Omega)\left(L^{2}(\Gamma)\right)$, temos

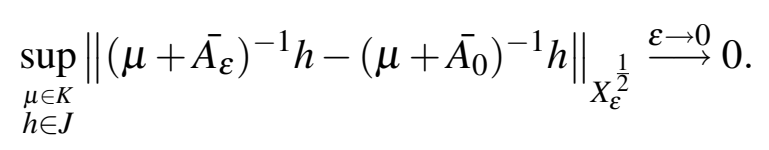

A cada ponto isolado $\mu \in \sigma\left(-\bar{A}_{0}\right)$, associamos seu auto-espaço generalizado

$$
W\left(\mu,-\bar{A}_{0}\right)=Q_{0}(\mu) X
$$

onde

$$
Q_{0}(\mu)=\frac{1}{2 \pi i} \int_{|z-\mu|=\delta}\left(z+\bar{A}_{0}\right)^{-1} d z
$$

e $\delta$ é escolhido de modo que não exista nenhum ponto de $\sigma\left(-\bar{A}_{0}\right)$ no disco $\{z \in \mathbb{C}:|z-\mu| \leqslant \delta\}$. Denotamos por $W\left(\mu,-\bar{A}_{\varepsilon}\right)=Q_{\varepsilon}(\mu) X$, onde

$$
Q_{\varepsilon}(\mu)=\frac{1}{2 \pi i} \int_{|z-\mu|=\delta}\left(z+\bar{A}_{\varepsilon}\right)^{-1} d z .
$$

Como nos Teoremas 3.6 e 3.9 , nosso próximo resultado garante que o espectro de $-\bar{A}_{\mathcal{\varepsilon}}$, para $\varepsilon$ pequeno, se aproxima do espectro de $-\bar{A}_{0}$.

Como $A_{\varepsilon}$ é setorial, tem resolvente compacto e $h^{\prime}\left(u_{*}^{\varepsilon}\right) \in \mathscr{L}\left(X_{\mathcal{\varepsilon}}^{\frac{1}{2}}, X_{0}^{\frac{-s}{2}}\right)$, então o operador $\bar{A}_{\mathcal{\varepsilon}}$ é setorial com resolvente compacto. Desta forma, os espectros de $-\overline{A_{\varepsilon}}, \varepsilon \in\left[0, \varepsilon_{0}\right]$ consistem apenas de autovalores isolados. 
Teorema 4.13. As seguintes afirmações são válidas:

(i) para todo $\mu_{0} \in \sigma\left(-\bar{A}_{0}\right)$, existem seqüências $\varepsilon_{n} \rightarrow 0$ e $\left\{\mu_{n}\right\}, \mu_{n} \in \sigma\left(-\bar{A}_{\varepsilon_{n}}\right), n \in \mathbb{N}$, tais que $\mu_{n} \rightarrow \mu_{0}$ quando $n \rightarrow \infty$.

(ii) se para alguma seqüência $\varepsilon_{n} \rightarrow 0$, e alguma seqüência $\left\{\mu_{n}\right\}, \mu_{n} \in \sigma\left(-\bar{A}_{\varepsilon_{n}}\right), n \in \mathbb{N}$, temos $\mu_{n} \rightarrow \mu_{0}$ quando $n \rightarrow \infty$, então $\mu_{0} \in \sigma\left(-\bar{A}_{0}\right)$.

(iii) existe $\bar{\varepsilon}>0$ tal que $\operatorname{dim} W\left(\mu,-\bar{A}_{\varepsilon}\right)=\operatorname{dim} W\left(\mu,-\bar{A}_{0}\right)$, para todo $0<\varepsilon \leqslant \bar{\varepsilon}$.

(iv) para todo $u \in W\left(\mu_{0},-\bar{A}_{0}\right)$, existe uma seqüência $\left\{u_{\varepsilon}\right\}, u^{\varepsilon} \in W\left(\mu_{0},-\bar{A}_{\varepsilon}\right)$, tal que $u^{\varepsilon} \stackrel{\varepsilon \rightarrow 0}{\longrightarrow} u$.

(v) toda seqüência $\varepsilon_{n} \rightarrow 0,\left\{u_{n}\right\}, u_{n} \in W\left(\mu,-\bar{A}_{\varepsilon_{n}}\right), n \in \mathbb{N}$, com $\left\|u_{n}\right\|_{X}=1$ tem uma subseqüência convergente e todo ponto limite desta seqüência está em $W\left(\mu_{0},-\bar{A}_{0}\right)$.

Proposição 4.14. Suponhamos que o problema $\left(\underline{\mathrm{E}_{0}}\right)$ tenha uma solução $u_{*}$ e que $0 \notin \sigma\left(A_{0}-\right.$ $\left.h^{\prime}\left(u_{*}\right)\right)$. Então existem $\bar{\varepsilon}>0$ e $\delta>0$ tais que o problema $\left(\mathrm{E}_{\mathcal{\varepsilon}}\right)$ possui exatamente uma solução, $u_{*}^{\varepsilon}$, em $\left\{w^{\varepsilon} \in X_{\varepsilon}^{\frac{1}{2}}:\left\|w^{\varepsilon}-u_{*}\right\|_{X_{\varepsilon}^{\frac{1}{2}}} \leqslant \delta\right\}$, para $\varepsilon \in(0, \bar{\varepsilon}]$. Além disso, $\left\|u_{*}^{\varepsilon}-u_{*}\right\|_{X_{\varepsilon}^{\frac{1}{2}}} \rightarrow 0$ quando $\varepsilon \rightarrow 0$.

Demonstração. Nosso objetivo é mostrar que em uma vizinhança da solução $u_{*}$ de $\left(\mathrm{E}_{0}\right)$ existe uma única solução $u_{*}^{\varepsilon}$ de $\left(\mathrm{E}_{\varepsilon}\right)$. Considerando a seqüência constante $u_{\varepsilon}=u^{*}$, podemos usar o Lema $4.10 \operatorname{com} \theta=\frac{1}{2}$, para garantir a existência de uma constante $C_{1}>0$ de tal maneira que

$$
\left\|\left(A_{\varepsilon}-h^{\prime}\left(u_{*}\right)\right)^{-1}\right\|_{\mathscr{L}\left(X, X_{\varepsilon}^{\frac{1}{2}}\right)} \leqslant C_{1}, \quad \operatorname{para} \varepsilon \in\left(0, \varepsilon_{0}\right] .
$$

Temos ainda que $\left|h^{\prime \prime}(u)\right| \leqslant C_{h}$, para todo $u \in X_{\varepsilon}^{\frac{1}{2}}$.

Observemos agora que mostrar que $u^{\varepsilon}$ é solução de $\left(\mathrm{E}_{\varepsilon}\right)$ é equivalente a mostrar que $u^{\varepsilon}$ é ponto fixo da aplicação

$$
\Phi_{\varepsilon}\left(u^{\varepsilon}\right)=-\left(A_{\varepsilon}-h^{\prime}\left(u_{*}\right)\right)^{-1}\left(h^{\prime}\left(u_{*}\right) u^{\varepsilon}-h\left(u^{\varepsilon}\right)\right) .
$$

Notemos que

$$
\begin{aligned}
\Phi_{\varepsilon}\left(u_{*}\right) & =-\left(A_{\varepsilon}-h^{\prime}\left(u_{*}\right)\right)^{-1}\left(h^{\prime}\left(u_{*}\right) u_{*}-h\left(u_{*}\right)\right) \\
& =-\left(A_{\varepsilon}-h^{\prime}\left(u_{*}\right)\right)^{-1}\left(h^{\prime}\left(u_{*}\right) u_{*}-A u_{*}\right) \\
& =\left(A_{\varepsilon}-h^{\prime}\left(u_{*}\right)\right)^{-1}\left(A-h^{\prime}\left(u_{*}\right)\right) u_{*} \rightarrow u_{*}
\end{aligned}
$$


quando $\varepsilon \rightarrow 0$.

Para mostrarmos que existe $\delta>0$ de tal forma que $\Phi_{\varepsilon}: \bar{B}_{X_{\varepsilon}^{\frac{1}{2}}}\left(u_{*}, \delta\right) \rightarrow \bar{B}_{X_{\varepsilon}^{\frac{1}{2}}}\left(u_{*}, \delta\right)$ é uma contração, procedemos de forma inteiramente análoga à (4.5), obtendo

$$
\begin{aligned}
\left\|\Phi_{\varepsilon}\left(u^{\varepsilon}\right)-\Phi_{\varepsilon}\left(v^{\varepsilon}\right)\right\|_{X_{\varepsilon}^{\frac{1}{2}}} & \leqslant C_{2}\left\|\left(\theta u^{\varepsilon}-(1-\theta) v^{\varepsilon}\right)-u_{*}\right\|_{X_{\varepsilon}^{\frac{1}{2}}}\left\|u^{\varepsilon}-v^{\varepsilon}\right\|_{X_{\varepsilon}^{\frac{1}{2}}} \\
& \leqslant 2 C_{2} \delta\left\|u^{\varepsilon}-v^{\varepsilon}\right\|_{X_{\varepsilon}^{\frac{1}{2}}},
\end{aligned}
$$

para quaisquer $u^{\varepsilon}, v^{\varepsilon} \in B=\bar{B}_{X_{\varepsilon}^{\frac{1}{2}}}\left(u_{*}, \delta\right)$. Basta, portanto, escolhermos $\delta>0$ tal que $2 C_{2} \delta<\frac{1}{2}$. Além disso, se $u^{\varepsilon} \in B$, então

$$
\begin{aligned}
\left\|\Phi\left(u^{\varepsilon}\right)-u_{*}\right\|_{X_{\varepsilon}^{\frac{1}{2}}} & \leqslant\left\|\Phi_{\varepsilon}\left(u^{\varepsilon}\right)-\Phi_{\varepsilon}\left(u_{*}\right)\right\|_{H^{1}(\Omega)}+\left\|\Phi_{\varepsilon}\left(u_{*}\right)-u_{*}\right\|_{X_{\varepsilon}^{\frac{1}{2}}} \\
& \leqslant \frac{1}{2}\left\|u^{\varepsilon}-u_{*}\right\|_{X_{\varepsilon}^{\frac{1}{2}}}+\left\|\Phi_{\varepsilon}\left(u_{*}\right)-u_{*}\right\|_{X_{\varepsilon}^{\frac{1}{2}}} \cdot
\end{aligned}
$$

De (4.13) segue que existe $\bar{\varepsilon}$ tal que

$$
\left\|\Phi_{\varepsilon}\left(u_{*}\right)-u_{*}\right\|_{X_{\varepsilon}^{\frac{1}{2}}} \leqslant \frac{\delta}{2},
$$

e assim, se $u^{\varepsilon} \in B$, obtemos

$$
\left\|\Phi_{\varepsilon}\left(u_{*}\right)-u_{*}\right\|_{X_{\varepsilon}^{\frac{1}{2}}} \leqslant \frac{\delta}{2}+\frac{\delta}{2}=\delta .
$$

Logo, $\Phi_{\varepsilon}(B) \subset B$ e $\Phi_{\varepsilon}: B \rightarrow B$ é uma contração, para todo $\varepsilon \in(0, \bar{\varepsilon}]$, de modo que existe um único ponto fixo de $\Phi_{\varepsilon}$ em $B$, que chamaremos de $u_{*}^{\varepsilon}$. Finalmente, vejamos que $u_{*}^{\varepsilon} \rightarrow u_{*}$ em $X_{\varepsilon}^{\frac{1}{2}}$, quando $\varepsilon \rightarrow 0$. De fato,

$$
\begin{aligned}
\left\|u_{*}^{\varepsilon}-u_{*}\right\|_{X_{\varepsilon}^{\frac{1}{2}}} & =\left\|\Phi_{\varepsilon}\left(u_{*}^{\varepsilon}\right)-u_{*}\right\|_{X_{\varepsilon}^{\frac{1}{2}}} \leqslant\left\|\Phi_{\varepsilon}\left(u_{*}^{\varepsilon}\right)-\Phi_{\varepsilon}\left(u_{*}\right)\right\|_{X_{\varepsilon}^{\frac{1}{2}}}+\left\|\Phi_{\varepsilon}\left(u_{*}\right)-u_{*}\right\|_{X_{\varepsilon}^{\frac{1}{2}}} \\
& \leqslant \frac{1}{2}\left\|u_{*}^{\varepsilon}-u_{*}\right\|_{X_{\varepsilon}^{\frac{1}{2}}}+\left\|\Phi_{\varepsilon}\left(u_{*}\right)-u_{*}\right\|_{X_{\varepsilon}^{\frac{1}{2}}},
\end{aligned}
$$

de maneira que $\left\|u_{*}^{\varepsilon}-u_{*}\right\|_{X_{\varepsilon}^{\frac{1}{2}}} \leqslant 2\left\|\Phi_{\varepsilon}\left(u_{*}\right)-u_{*}\right\|_{X_{\varepsilon}^{\frac{1}{2}}} \rightarrow 0$ quando $\varepsilon \rightarrow 0$.

Corolário 4.15. Suponhamos que as hipóteses da Proposição 4.14 estejam satisfeitas. Assumamos que $\left(\underline{\mathrm{E}_{0}}\right)$ tenha exatamente $n_{0}$ soluções $u_{1}, \ldots, u_{n_{0}}$, e que $0 \notin \sigma\left(A_{0}-h^{\prime}\left(u_{j}\right)\right)$, para $j=$ $1, \ldots, n_{0}$. Então existe $\bar{\varepsilon}$ suficientemente pequeno de tal modo que, para $\varepsilon \in(0, \bar{\varepsilon}]$, o problema $\left(\underline{\mathrm{E}_{\varepsilon}}\right)$ tem exatamente $n_{0}$ soluções $u_{1}^{\varepsilon}, \ldots, u_{n_{0}}^{\varepsilon}$. Além disso, para cada $j=1, \ldots, n_{0}$, temos

$$
\left\|u_{j}^{\varepsilon}-u_{j}\right\|_{X_{\varepsilon}^{\frac{1}{2}}} \rightarrow 0 \quad \text { quando } \varepsilon \rightarrow 0 \text {. }
$$


Demonstração. Da Proposição 4.7 segue que, para $\varepsilon$ suficientemente pequeno, toda solução $u^{\varepsilon}$ do problema $\left(\mathrm{E}_{\varepsilon}\right)$ está em uma vizinhança do conjunto de equilíbrio de $\left(\mathrm{E}_{0}\right)$. Além disso, pela Proposição 4.14, em uma vizinhança de $u_{j}$ existe apenas uma solução de $\left(\underline{\mathrm{E}_{\varepsilon}}\right), u_{j}^{\varepsilon}$, que converge para $u_{j}, j=1, \ldots, n_{0}$.

Denotemos por $\sigma_{\varepsilon}^{+}=\left\{\mu \in \sigma\left(-\bar{A}_{\varepsilon}\right): \operatorname{Re} \mu>0\right\}, \varepsilon \in\left[0, \varepsilon_{0}\right]$. Seja $Q_{0}^{+}\left(\sigma^{+}\right)$a projeção determinada por $\sigma_{0}^{+}$, isto é,

$$
Q_{0}^{+}\left(\sigma_{0}^{+}\right)=\frac{1}{2 \pi i} \int_{\Gamma^{+}}\left(\mu+\bar{A}_{0}\right)^{-1} d \mu
$$

onde $\Gamma^{+}$é uma curva em $\left\{\mu \in \rho\left(-A_{0}\right): \operatorname{Re} \mu>0\right\}$, que envolve $\sigma_{0}^{+}$. Do Lema ??, segue a existência de um $\varepsilon_{\Gamma}$ tal que $\Gamma^{+} \subset \rho\left(-\bar{A}_{\varepsilon}\right)$ for $\varepsilon \in\left(0, \varepsilon_{\Gamma}^{+}\right]$. Seja $Q_{\varepsilon}^{+}\left(\sigma_{\varepsilon}^{+},-\bar{A}_{\varepsilon}\right)$ projeção dada por

$$
Q_{\varepsilon}^{+}\left(\sigma_{\varepsilon}^{+}\right)=\frac{1}{2 \pi i} \int_{\Gamma^{+}}\left(\mu+\bar{A}_{\varepsilon}\right)^{-1} d \mu
$$

Consideremos $-\bar{A}_{\mathcal{\varepsilon}}^{+} \mathrm{e}-\bar{A}_{\varepsilon}^{-}$as restrições de $-\bar{A}_{\varepsilon}$ a $W_{\varepsilon}^{+}=Q_{\varepsilon}^{+}\left(\sigma_{\varepsilon}^{+}\right) X$ e ao espaço $W_{\varepsilon}^{-}=(I-$ $\left.Q_{\varepsilon}^{+}\left(\sigma_{\varepsilon}^{+}\right)\right) X$, respectivamente. Denotemos $-\bar{A}_{\varepsilon}^{ \pm}=-\left.\bar{A}_{\varepsilon}\right|_{W_{\varepsilon}^{ \pm}}, \varepsilon \in\left[0, \varepsilon_{0}\right]$.

Teorema 4.16. São válidas as seguintes afirmações:

(i) existe um $\varepsilon_{0}>0$ tal que $\operatorname{dim} W\left(\sigma_{\varepsilon}^{+},-{\overline{A_{\varepsilon}^{+}}}^{-}\right)=\operatorname{dim} W\left({\sigma_{0}^{+}}^{+},{\overline{A_{0}}}^{+}\right)$, para todo $0<\varepsilon \leqslant \varepsilon_{0}$.

(ii) para todo $u \in W\left({\sigma_{0}^{+}}^{+},{\overline{A_{0}}}^{+}\right)$, existe uma seqüência $\left\{u_{\varepsilon}\right\}, u^{\varepsilon} \in W\left(\sigma_{\varepsilon}^{+},-\overline{A_{\varepsilon}}\right)$, tal que $u^{\varepsilon} \stackrel{\varepsilon \rightarrow 0}{\longrightarrow}$ $u$.

(iii) toda seqüência $\left\{u_{n}\right\}, u_{n} \in W\left(\sigma_{\varepsilon}^{+},-\bar{A}_{\varepsilon_{n}}\right), n \in \mathbb{N}, \varepsilon_{n} \rightarrow 0$, com $\left\|u_{n}\right\|_{X}=1$ possui uma subseqüência convergente e todo ponto limite desta seqüência está em $W\left(\sigma_{0}^{+},-{\overline{A_{0}}}^{+}\right)$.

(iv) existem $\beta>0$ e $\varepsilon_{\beta}>0$ tais que $\sigma\left(-\bar{A}_{\varepsilon}^{+}\right) \cap\{\mu \in \mathbb{C}:|\operatorname{Re} \mu|>\beta\}=\varnothing, \varepsilon \in\left(0, \varepsilon_{\beta}\right]$.

(v) existem $\frac{\pi}{2}>\phi_{\beta}>0, \omega_{\beta}>0$ e $\varepsilon_{\beta}>0$ tais que $\Sigma_{-\omega_{\beta}, \phi_{\beta}} \subset \rho\left(-\bar{A}_{\varepsilon}^{-}\right), \varepsilon \in\left(0, \varepsilon_{\beta}\right] e$

$$
\left\|\left(\mu+\bar{A}_{\varepsilon}^{-}\right)^{-1}\right\|_{\mathscr{L}(X)} \leqslant \frac{M_{\beta}}{\left|\mu-\omega_{\beta}\right|}, \text { para todo } \mu \in \Sigma_{-\omega_{\beta}, \phi_{\beta}} \text { e para todo } \varepsilon \in\left(0, \varepsilon_{\beta}\right]
$$




\subsection{Continuidade das variedades instáveis}

Recordemos que estamos assumindo que todas as soluções de equilíbrio de (2.8) são hiperbólicas, ou seja, que cada solução de $\left(\mathrm{E}_{0}\right)$ é tal que o operador $-\bar{A}_{0}$ tem espectro $\sigma\left(-\bar{A}_{0}\right)$ disjunto do eixo imaginário.

Já sabemos que $\sigma_{0}^{+}=\sigma\left(-\bar{A}_{0}\right) \cap\{z \in \mathbb{C}: \operatorname{Re} z>0\}$ consiste de um número finito (possivelmente zero) de autovalores com multiplicidades finitas, e existem uma constante $\beta>0$ e uma projeção $Q_{0}^{+}$de posto finito sobre $\sigma_{0}^{+}$tal que

$$
\begin{aligned}
& \left\|e^{-\bar{A}_{0} t} Q_{0}^{+}\right\|_{\mathscr{L}\left(X_{0}^{\frac{-s}{2}}\right)} \leqslant M e^{\beta t}, \quad t \leqslant 0 \\
& \left\|e^{-\bar{A}_{0} t}\left(I-Q_{0}^{+}\right)\right\|_{\mathscr{L}\left(X_{0}^{\frac{-s}{2}}, X_{0}^{\frac{1}{2}}\right)} \leqslant M t^{-\left(\frac{1+s}{2}\right)} e^{-\beta t}, \quad t>0 .
\end{aligned}
$$

Como vimos na seção anterior, para $\varepsilon$ suficientemente pequeno, existe uma solução $u_{*}^{\varepsilon}$ de $\left(\underline{\mathrm{E}_{\varepsilon}}\right)$, tal que $u_{*}^{\varepsilon} \rightarrow u_{*}$ em $X_{\varepsilon}^{\frac{1}{2}}$.

Proposição 4.17. Sejam $u_{*}^{\varepsilon}$ solução de $\left(\underline{\mathrm{E}_{\varepsilon}}\right)$ e $u_{*}$ solução de $\left(\underline{\mathrm{E}_{0}}\right)$ tais que $u_{*}^{\varepsilon} \rightarrow u_{*}$. Se $\bar{A}_{\varepsilon}=$ $A_{\varepsilon}-h^{\prime}\left(u_{*}^{\varepsilon}\right)$, então existe um $\bar{\varepsilon}>0$ tal que $\sigma\left(-\bar{A}_{\varepsilon}\right)$ não intercepta o eixo imaginário, para $0 \leqslant \varepsilon \leqslant \bar{\varepsilon}$, e $\left\|\left(A_{\varepsilon}-h^{\prime}\left(u_{*}^{\varepsilon}\right)\right)^{-1}\right\| \leqslant C$, com $C$ independente de $\varepsilon$. Além disso, se $Q_{\varepsilon}^{+}$denota a projeção definida pelo conjunto espectral $\sigma_{\varepsilon}^{+}=\left\{\mu \in \sigma\left(-\bar{A}_{\varepsilon}\right): \operatorname{Re} \mu>0\right\}$, então $Q_{\varepsilon}^{+}$converge compactamente para $Q_{0}^{+}$quando $\varepsilon \rightarrow 0$ (e então posto $\left(\mathrm{Q}_{\varepsilon}^{+}\right)=\operatorname{posto}\left(\mathrm{Q}_{0}^{+}\right)$, para $0<\varepsilon \leqslant \bar{\varepsilon}$ ), e a família de conjuntos $\sigma_{\varepsilon}^{+}$é semicontínua inferior e superiormente em $\varepsilon=0$.

Proposição 4.18. Para $\varepsilon$ suficientemente pequeno, o ponto de equilíbrio $u_{*}^{\varepsilon}$ de $\left(\overline{\mathrm{E}_{\mathcal{E}}}\right)$ é hiperbólico e existe $\beta>0$ tal que, se $Q_{\varepsilon}^{+}$é a projeção determinada por $\sigma_{+}^{\varepsilon}$, então

$$
\begin{aligned}
& \left\|e^{-\bar{A}_{\varepsilon} t} Q_{\varepsilon}^{+}\right\|_{\mathscr{L}\left(X_{\varepsilon}^{\frac{-s}{2}}\right)} \leqslant M e^{\beta t}, \quad t \leqslant 0 \\
& \left\|e^{-\bar{A}_{\varepsilon} t}\left(I-Q_{\varepsilon}^{+}\right)\right\|_{\mathscr{L}\left(X_{\varepsilon} \frac{-s}{2}, X_{\varepsilon}^{\frac{1}{2}}\right)} \leqslant M t^{-\left(\frac{1+s}{2}\right)} e^{-\beta t}, \quad t>0 .
\end{aligned}
$$

Assumamos que $\left(\mathrm{E}_{0}\right)$ tenha apenas um número finito de soluções $u_{1}, \cdots, u_{m}$. Nesta seção mostramos que as variedades instáveis locais de $u_{k}$, para $k=1, \ldots, m$ fixado, são contínuas. A existência destas variedades segue da teoria canônica de variedades invariantes, veja [15]. 
Mostramos que as variedades instáveis estão próximas para $\varepsilon$ pequeno. Para tanto, usamos os resultados de convergência da parte linear, obtidos nos capítulos precedentes.

Pela Proposição 4.14, temos que $\left\|u_{*}^{\varepsilon}-u_{*}\right\|_{X_{\varepsilon}^{\frac{1}{2}}} \rightarrow 0$ quando $\varepsilon \rightarrow 0$. Segue então da Proposição 4.17 que $\sigma\left(-\bar{A}_{\varepsilon}\right)$ se comporta continuamente quando $\varepsilon \rightarrow 0$.

Para trabalharmos em uma vizinhança do ponto de equilíbrio $u_{*}^{\varepsilon}$, reescrevemos o problema (2.6) para $w^{\varepsilon}=u^{\varepsilon}-u_{*}^{\varepsilon}$, obtendo

$$
\dot{w}^{\varepsilon}+\bar{A}_{\varepsilon} w^{\varepsilon}=h\left(w^{\varepsilon}+u_{*}^{\varepsilon}\right)-h\left(u_{*}^{\varepsilon}\right)-h^{\prime}\left(u_{*}^{\varepsilon}\right) w^{\varepsilon},
$$

onde $\bar{A}_{\varepsilon}=A_{\varepsilon}-h^{\prime}\left(u_{*}^{\varepsilon}\right)$.

Para obtermos as projeções $Q_{\varepsilon}^{+}$procedemos do seguinte modo: denotamos por $\left\{\mu_{i}^{\varepsilon}\right\}_{i=1}^{\infty}$ os autovalores de $\bar{A}_{\varepsilon}, \varepsilon \in\left[0, \varepsilon_{0}\right]$ (ordenados e contando suas multiplicidades), e por $\left\{\phi_{i}^{\varepsilon}\right\}_{i=1}^{\infty}$ um correspondente sistema ortonormal de autofunções. Ordenamos os autovalores de tal maneira que $\mu_{1}^{\varepsilon} \geqslant \ldots \geqslant \mu_{k}^{\varepsilon}>\omega>0>-\omega>\mu_{k+1}^{\varepsilon} \geqslant \mu_{k+2}^{\varepsilon} \geqslant \ldots, \varepsilon \in[0, \bar{\varepsilon}]$, para algum $\omega>0$ e $\bar{\varepsilon} \in$ $\left(0, \varepsilon_{0}\right]$. Consideramos $W_{\varepsilon}=\left[\phi_{1}^{\varepsilon}, \ldots, \phi_{k}^{\varepsilon}\right]$ e $W_{\varepsilon}^{\perp}=\left\{\psi \in X_{\varepsilon}^{\frac{1}{2}}: \int_{\Omega} \psi \phi=0\right.$, para qualquer $\left.\phi \in W_{\varepsilon}\right\}$. Sejam $Q_{\varepsilon}^{+}: X_{\varepsilon}^{\frac{1}{2}} \rightarrow X_{\varepsilon}^{\frac{1}{2}}$ as projeções ortogonais sobre $W_{\varepsilon}, \varepsilon \in\left[0, \varepsilon_{0}\right]$,

$$
Q_{\varepsilon}^{+} \psi=\sum_{i=1}^{k}\left(\int_{\Omega} \psi \phi_{i}^{\varepsilon}\right) \phi_{i}^{\varepsilon}
$$

Se $\psi \in W_{\varepsilon}$, então $\psi=\sum_{i=1}^{k}\left(\int_{\Omega} \psi \phi_{i}^{\varepsilon}\right) \phi_{i}^{\varepsilon}$ e

$$
\|\psi\|_{X_{\varepsilon}^{\frac{1}{2}}}=\left(\sum_{i=1}^{k}\left(1+\mu_{i}^{\varepsilon}\right)\left(\int_{\Omega} \psi \phi_{i}^{\varepsilon}\right)^{2}\right)^{\frac{1}{2}} .
$$

Como os autovalores $\mu_{i}^{\varepsilon} \rightarrow \mu_{i}^{0}$ quando $\varepsilon \rightarrow 0$ para $1 \leqslant i<\infty$, segue que $W_{\varepsilon}$ é isomorfo a $W_{0}$, para $\varepsilon \in(0, \bar{\varepsilon}]$. Ademais, $W_{\varepsilon}, \varepsilon \in[0, \bar{\varepsilon}]$, é isomorfo a $\mathbb{R}^{k}$ através do isomorfismo $I_{\varepsilon}$ dado por

$$
W_{\varepsilon} \ni \psi \mapsto\left(\int_{\Omega} \psi \phi_{1}^{\varepsilon}, \ldots, \int_{\Omega} \psi \phi_{k}^{\varepsilon}\right) \in \mathbb{R}^{k}
$$

Notemos que $I_{\varepsilon}$ é um operador limitado com inversa $I_{\mathcal{\varepsilon}}^{-1}$ limitada e que as normas de $I_{\mathcal{\varepsilon}}$ e $I_{\mathcal{\varepsilon}}^{-1}$ são uniformemente limitadas, para $\varepsilon \in[0, \bar{\varepsilon}]$. 
Este fato nos permite decompor o espaço $X_{\varepsilon}^{\frac{1}{2}}$ em dois espaços, $\mathbb{R}^{k}$ e $\left(I-Q_{\varepsilon}^{+}\right) X_{\varepsilon}^{\frac{1}{2}}$. Esta decomposição do espaço induz uma decomposição da solução $w^{\varepsilon}$ de (4.17), da seguinte maneira: $w^{\varepsilon}=v^{\varepsilon}+z^{\varepsilon}, \operatorname{com} v^{\varepsilon}=\sum_{i=1}^{k} v_{i} \phi_{i}^{\varepsilon}, v_{i}=\int_{\Omega} w \phi_{i}^{\varepsilon}$ e $z^{\varepsilon}=\left(I-Q_{\varepsilon}^{+}\right) w^{\varepsilon}$.

Assim, como $\dot{v}^{\varepsilon}=Q_{\mathcal{\varepsilon}}^{+} \dot{w}^{\varepsilon}, \dot{z}^{\varepsilon}=\left(I-Q_{\varepsilon}^{+}\right) \dot{w}^{\varepsilon}$ e $Q_{\varepsilon}^{+},\left(I-Q_{\varepsilon}^{+}\right)$comutam com $\overline{A_{\varepsilon}}$, obtemos

$$
\dot{v}_{i}+\mu_{i}^{\varepsilon} v_{i}=\int_{\Omega}\left[\left(h\left(v^{\varepsilon}+z^{\varepsilon}+u_{*}^{\varepsilon}\right)-h\left(u_{*}^{\varepsilon}\right)-h^{\prime}\left(u_{*}^{\varepsilon}\right)\left(v^{\varepsilon}+z^{\varepsilon}\right)\right)\right] \phi_{i}^{\varepsilon}
$$

e

$$
\begin{aligned}
\dot{z}^{\varepsilon}+\bar{A}_{\varepsilon} z^{\varepsilon} & =\left(I-Q_{\varepsilon}^{+}\right)\left(h\left(v^{\varepsilon}+z^{\varepsilon}+u_{*}^{\varepsilon}\right)-h\left(u_{*}^{\varepsilon}\right)-h^{\prime}\left(u_{*}^{\varepsilon}\right)\left(v^{\varepsilon}+z^{\varepsilon}\right)\right) \\
& =h\left(w^{\varepsilon}+u_{*}^{\varepsilon}\right)-h\left(u_{*}^{\varepsilon}\right)-h^{\prime}\left(u_{*}^{\varepsilon}\right)\left(w^{\varepsilon}\right) \\
& -\sum_{i=1}^{k}\left(\int_{\Omega}\left[h\left(w^{\varepsilon}+u_{*}^{\varepsilon}\right)-h\left(u_{*}^{\varepsilon}\right)-h^{\prime}\left(u_{*}^{\varepsilon}\right)\left(w^{\varepsilon}\right)\right] \phi_{i}^{\varepsilon}\right) \phi_{i}^{\varepsilon} .
\end{aligned}
$$

Denotando $v=\left(v_{1}, \ldots, v_{k}\right)^{t}$ e $H_{\varepsilon}(v, z)=\left(H_{1}(v, z), \ldots, H_{k}(v, z)\right)^{t}$, onde

$$
H_{j}(v, z)=\int_{\Omega}\left[h\left(\sum_{i=1}^{k} v_{i} \phi_{i}^{\varepsilon}+z^{\varepsilon}+u_{*}^{\varepsilon}\right)-h\left(u_{*}^{\varepsilon}\right)-h^{\prime}\left(u_{*}^{\varepsilon}\right)\left(\sum_{i=1}^{k} v_{i} \phi_{i}^{\varepsilon}+z^{\varepsilon}\right)\right] \phi_{j}^{\varepsilon}
$$

e também

$$
G_{\varepsilon}(v, z)=\left(I-Q_{\varepsilon}^{+}\right)\left(h\left(v^{\varepsilon}+z^{\varepsilon}+u_{*}^{\varepsilon}\right)-h\left(u_{*}^{\varepsilon}\right)-h^{\prime}\left(u_{*}^{\varepsilon}\right)\left(v^{\varepsilon}+z^{\varepsilon}\right)\right),
$$

resulta que $H_{\varepsilon}(0,0)=0=G_{\varepsilon}(0,0)$. Além disso, $H_{\varepsilon}$ e $G_{\varepsilon}$ são continuamente diferenciáveis e satisfazem $\frac{\partial H_{\varepsilon}}{\partial v}(0,0)=0=\frac{\partial G_{\varepsilon}}{\partial v}(0,0)$. Como as projeções $Q_{\varepsilon}^{+}, \varepsilon \in\left(0, \varepsilon_{0}\right]$, são uniformemente limitadas e a função $h$ não depende de $\varepsilon$ segue que, dado $\rho>0$, existem $\bar{\varepsilon}>0$ e $\delta>0$ de tal forma que se $\|v\|_{\mathbb{R}^{k}}+\|z\|_{X_{\varepsilon}^{\frac{1}{2}}}<\delta$ e $\varepsilon \leqslant \bar{\varepsilon}$, então

$$
\begin{aligned}
& \left\|H_{\varepsilon}(v, z)\right\|_{\mathbb{R}^{k}} \leqslant \rho, \\
& \left\|G_{\varepsilon}(v, z)\right\|_{X_{\varepsilon}^{-\frac{s}{2}}} \leqslant \rho, \\
& \left\|H_{\mathcal{\varepsilon}}(v, z)-H_{\varepsilon}(\tilde{v}, \tilde{z})\right\|_{\mathbb{R}^{k}} \leqslant \rho\left(\|v-\tilde{v}\|_{\mathbb{R}^{k}}+\|z-\tilde{z}\|_{X_{\varepsilon}^{\frac{1}{2}}}\right), \\
& \left\|G_{\varepsilon}(v, z)-G_{\varepsilon}(\tilde{v}, \tilde{z})\right\|_{X_{\varepsilon}^{-\frac{s}{2}}} \leqslant \rho\left(\|v-\tilde{v}\|_{\mathbb{R}^{k}}+\|z-\tilde{z}\|_{X_{\varepsilon}^{\frac{1}{2}}}\right) .
\end{aligned}
$$

Procedendo como na demonstração do Lema 4.9 podemos escolher $\rho$ e $\delta$ uniformemente para $\varepsilon \in(0, \bar{\varepsilon}]$ satisfazendo as desigualdades acima. Este é o ponto chave para obtermos que as 
variedades instáveis locais estão definidas em uma pequena vizinhança do ponto de equilíbrio $u_{*}^{\varepsilon}$, uniformemente para $\varepsilon \leqslant \bar{\varepsilon}$.

Podemos estender $H_{\varepsilon}$ e $G_{\varepsilon}$ fora de $B_{X_{\varepsilon}^{\frac{1}{2}}}\left(u_{*}^{\varepsilon}, \delta\right)$ de tal forma que as estimativas em (4.20) permaneçam válidas para quaisquer $v^{\varepsilon} \in W$ e $z^{\varepsilon} \in W_{\varepsilon}^{\perp}$.

Observação 1. O procedimento para se estender uma função $g$ definida em uma bola de raio $\frac{1}{n}$ de tal maneira que ela se se torne globalmente de Lipschitz contínua, sem alterar sua constante de Lipschitz é o seguinte: dada uma função $g: V \times Z \rightarrow Y, V, Z$ e $Y$ espaços de Banach, definimos

$$
g_{n}(v, z)=\left\{\begin{array}{l}
g(v, z), \quad\|v+z\| \leqslant \frac{1}{n} \\
g\left(\frac{v}{n\|v+z\|}, \frac{z}{n\|v+z\|}\right), \quad\|v+z\|>\frac{1}{n}
\end{array}\right.
$$

As constantes de Lipschitz para $g_{n}$ são justamente as constantes de Lipschitz para g quando restrita a $B_{\frac{1}{n}}$, ou seja, $\left\|g_{n}\left(v_{1}, z_{1}\right)-g_{n}\left(v_{2}, z_{2}\right)\right\| \leqslant L_{g_{n}}\left(\left\|v_{1}-v_{2}\right\|+\left\|z_{1}-z_{2}\right\|\right)$ e $L_{g_{n}}$ é a constante de Lipschitz de g na bola.

Este procedimento aplicado a $H_{\varepsilon}$ e $G_{\varepsilon}$ nos fornece as extensões desejadas para $H_{\varepsilon}$ e $G_{\varepsilon}$.

Denotemos $\bar{A}_{\varepsilon}^{+}:=\operatorname{diag}\left(\mu_{1}^{\varepsilon}, \ldots, \mu_{k}^{\varepsilon}\right)$ e $\bar{A}_{\varepsilon}^{-}:={\overline{A_{\varepsilon}}}_{\left(I-Q_{\varepsilon}^{+}\right) X_{\varepsilon}^{\frac{1}{2}}}$. Podemos então reescrever a equação (4.17) como

$$
\begin{cases}\dot{v}^{\varepsilon}+\bar{A}_{\varepsilon}^{+} v^{\varepsilon} & =H_{\varepsilon}\left(v^{\varepsilon}, z^{\varepsilon}\right) \\ \dot{z}^{\varepsilon}+\bar{A}_{\varepsilon}^{-} z^{\varepsilon} & =G_{\varepsilon}\left(v^{\varepsilon}, z^{\varepsilon}\right)\end{cases}
$$

onde $H_{\varepsilon}$ e $G_{\varepsilon}$ satisfazem (4.20), par quaisquer $v^{\varepsilon} \in \mathbb{R}^{k}$ e $z^{\varepsilon} \in\left(I-Q_{\varepsilon}^{+}\right) X_{\varepsilon}^{\frac{1}{2}}$.

Além disso, para apropriadas constantes positivas $M, \beta$, independentes de $\varepsilon, \varepsilon \leqslant \bar{\varepsilon}$, temos

$$
\begin{aligned}
& \left\|e^{-\bar{A}_{\varepsilon}^{-} t} z\right\|_{X_{\varepsilon}^{\frac{1}{2}}} \leqslant M e^{-\beta t}\|z\|_{X_{\varepsilon}^{\frac{1}{2}}}, \quad t \geqslant 0, \\
& \left\|e^{-\bar{A}_{\varepsilon}^{-} t} z\right\|_{X_{\varepsilon}^{\frac{1}{2}}} \leqslant M t^{-\left(\frac{1+s}{2}\right)} e^{-\beta t}\|z\|_{X_{\varepsilon}^{-\frac{s}{2}}}, \quad t>0, \\
& \left\|e^{-\bar{A}_{\varepsilon}^{+} t} v\right\|_{\mathbb{R}^{k}} \leqslant M e^{\beta t}\|v\|_{\mathbb{R}^{k}}, \quad t \leqslant 0 .
\end{aligned}
$$

Em seguida mostraremos que para $\rho>0$ escolhido apropriadamente, a variedade instável para $u_{*}^{\varepsilon}$ é dada localmente por

$$
\mathrm{W}_{\varepsilon}^{u}=\left\{(v, z): z=\mathscr{S}_{\varepsilon}^{*}(v), v \in \mathbb{R}^{k}\right\}
$$

onde $\mathscr{S}_{\varepsilon}^{*}: \mathbb{R}^{k} \rightarrow\left(I-Q_{\varepsilon}^{+}\right) X_{\varepsilon}^{\frac{1}{2}}$ é limitada e Lipschitz contínua. Além disso, para qualquer $R>0$,

$$
\sup _{v \in B_{\mathbb{R}^{k}}(0, R)}\left\|\mathscr{S}_{\varepsilon}^{*}(v)-\mathscr{S}_{0}^{*}(v)\right\|_{X_{\varepsilon}^{\frac{1}{2}}} \stackrel{\varepsilon \rightarrow 0}{\longrightarrow} 0 .
$$


Proposição 4.19. Assumamos que $u_{*}$ seja um equilíbrio hiperbólico de (2.8). Então, pela Proposição 4.14 o problema (2.6) tem uma única solução de equilíbrio, $u_{*}^{\varepsilon}$, próxima a $u_{*}$. Para $D>0, \Delta>0,0<\vartheta<1$ dados, seja $\rho_{0}>0$ de tal forma que, para todo $0<\rho \leqslant \rho_{0}$, sejam válidas as seguintes estimativas:

$$
\begin{aligned}
& \rho M \beta^{\frac{s-1}{2}} \Gamma\left(\frac{1-s}{2}\right) \leqslant D \\
& \rho M^{2}(1+\Delta)\left(\frac{\beta}{2}\right)^{\frac{s-1}{2}} \Gamma\left(\frac{1-s}{2}\right) \leqslant \Delta \\
& \frac{\beta}{2} \leqslant 2 \beta-\rho M(1+\Delta) \leqslant 2 \beta \\
& \rho M \beta^{\frac{s-1}{2}} \Gamma\left(\frac{1-s}{2}\right)\left[1+\frac{\rho M(1+\Delta)}{2 \beta-\rho M(1+\Delta)}\right] \leqslant \vartheta<1 \\
& \gamma=\beta-\left[\rho M+\frac{\rho^{2} M^{2}(1+\Delta)(1+M)}{2 \beta-\rho M(1+\Delta)}\right]>0 \\
& \rho M \beta^{\frac{s-1}{2}} \Gamma\left(\frac{1-s}{2}\right)+\left[\rho^{2} M^{2} \beta^{-1}(1+\Delta)\left(2 \beta-(\rho M(1+\Delta))^{\frac{s-1}{2}} \Gamma\left(\frac{1-s}{2}\right)\right] \leqslant \frac{1}{2}\right.
\end{aligned}
$$

Assumamos que, para $\rho$ escolhido como acima, as funções $H_{\varepsilon}$ e $G_{\varepsilon}$ satisfaçam (4.20) para qualquer $(v, z) \in \mathbb{R}^{k} \times\left(I-Q_{\varepsilon}^{+}\right) X_{\varepsilon}^{\frac{1}{2}}$. Então existe $\mathscr{S}_{\varepsilon}^{*}: \mathbb{R}^{k} \rightarrow\left(I-Q_{\varepsilon}^{+}\right) X_{\varepsilon}^{\frac{1}{2}}$ de tal maneira que

$$
\left\|\mathscr{S}_{\varepsilon}^{*}\right\|:=\sup _{v \in \mathbb{R}^{k}}\left\|\mathscr{S}_{\varepsilon}^{*}(v)\right\|_{X_{\varepsilon}^{\frac{1}{2}}} \leqslant D, \quad\left\|\mathscr{S}_{\varepsilon}^{*}(v)-\mathscr{S}_{\varepsilon}^{*}(\tilde{v})\right\|_{X_{\varepsilon}^{\frac{1}{2}}} \leqslant \Delta\|v-\tilde{v}\|_{\mathbb{R}^{k}}
$$

$e$

$$
\mathrm{W}_{\varepsilon}^{u}=\left\{(v, z): z=\mathscr{S}_{\varepsilon}^{*}(v), v \in \mathbb{R}^{k}\right\} .
$$

Além disso, para qualquer $R>0$,

$$
\sup _{v \in B_{\mathbb{R}^{k}}(0, R)}\left\|\mathscr{S}_{\varepsilon}^{*}(v)-\mathscr{S}_{0}^{*}(v)\right\|_{X_{\varepsilon}^{\frac{1}{2}}} \stackrel{\varepsilon \rightarrow 0}{\longrightarrow} 0
$$

Demonstração. Mostraremos primeiramente a existência da variedade invariante.

$\operatorname{Seja~} v^{\varepsilon}(t)=\psi\left(t, \tau, \eta, \mathscr{S}_{\varepsilon}\right)$ a solução de

$$
\dot{v}^{\varepsilon}+\bar{A}_{\mathcal{\varepsilon}}^{+} v^{\varepsilon}=H_{\mathcal{\varepsilon}}\left(v^{\varepsilon}, \mathscr{S}_{\mathcal{\varepsilon}}\left(v^{\varepsilon}\right)\right), \quad \text { para } t<\tau, v^{\varepsilon}(\tau)=\eta
$$

Consideremos

$$
\mathscr{X}=\left\{\mathscr{S}: \mathbb{R}^{k} \rightarrow\left(I-Q_{\varepsilon}^{+}\right) X_{\mathcal{\varepsilon}}^{\frac{1}{2}}:\|\mathscr{S}\| \leqslant D,\left\|\mathscr{S}_{\varepsilon}(v)-\mathscr{S}_{\varepsilon}(\tilde{v})\right\|_{X_{\varepsilon}^{\frac{1}{2}}} \leqslant \Delta\|v-\tilde{v}\|_{\mathbb{R}^{k}}\right\} .
$$


É fácil ver que $(\mathscr{X},\|\cdot \cdot\|)$ é um espaço métrico completo.

Seja $\mathscr{S}_{\varepsilon} \in \mathscr{X}$ e definamos $\Phi: \mathscr{X} \rightarrow \mathscr{X}$ por

$$
\Phi\left(\mathscr{S}_{\varepsilon}\right)(\eta)=\int_{-\infty}^{\tau} e^{-\bar{A}_{\bar{\varepsilon}}^{-}(\tau-r)} G_{\varepsilon}\left(v^{\varepsilon}(s), \mathscr{S}_{\varepsilon}\left(v^{\varepsilon}(s)\right)\right) d r
$$

Notemos que

$$
\left\|\Phi\left(\mathscr{S}_{\varepsilon}\right)(\cdot)\right\|_{X_{\varepsilon}^{\frac{1}{2}}} \leqslant \int_{-\infty}^{\tau} \rho M(\tau-r)^{-\left(\frac{1+s}{2}\right)} e^{-\beta(\tau-r)} d r=\rho M \beta^{\left(\frac{s-1}{2}\right)} \Gamma\left(\frac{1-s}{2}\right) \leqslant D .
$$

Suponhamos agora que $\mathscr{S}_{\varepsilon}, \tilde{\mathscr{S}}_{\varepsilon} \in \mathscr{X}$, que $\eta, \tilde{\eta} \in \mathbb{R}^{k}$ e denotemos $v^{\varepsilon}(t)=\psi\left(t, \tau, \eta, \mathscr{S}_{\varepsilon}\right)$, $\tilde{v}^{\varepsilon}(t)=\psi\left(t, \tau, \tilde{\eta}, \tilde{\mathscr{S}}_{\varepsilon}\right)$. Então

$$
v^{\varepsilon}(t)-\tilde{v}^{\varepsilon}(t)=e^{-\bar{A}_{\varepsilon}^{+}(t-\tau)}(\eta-\tilde{\eta})+\int_{\tau}^{t} e^{-\bar{A}_{\varepsilon}^{+}(t-r)}\left[H_{\mathcal{\varepsilon}}\left(v^{\varepsilon}, \mathscr{S}_{\varepsilon}\left(v^{\varepsilon}\right)\right)-H_{\varepsilon}\left(\tilde{v}^{\varepsilon}, \tilde{\mathscr{S}}_{\varepsilon}\left(\tilde{v}^{\varepsilon}\right)\right)\right] d r .
$$

Notemos agora que

$$
\begin{aligned}
\left\|v^{\varepsilon}(t)-\tilde{v}^{\varepsilon}(t)\right\|_{\mathbb{R}^{k} \leqslant} & M e^{\beta(t-\tau)}\|\eta-\tilde{\eta}\|_{\mathbb{R}^{k}} \\
& +M \int_{t}^{\tau} e^{\beta(t-r)}\left\|H_{\varepsilon}\left(v^{\varepsilon}, \mathscr{S}_{\varepsilon}\left(v^{\varepsilon}\right)\right)-H_{\varepsilon}\left(\tilde{v}^{\varepsilon}, \tilde{\mathscr{S}}_{\varepsilon}\left(\tilde{v}^{\varepsilon}\right)\right)\right\|_{\mathbb{R}^{k}} d r \\
\leqslant & M e^{\beta(t-\tau)}\|\eta-\tilde{\eta}\|_{\mathbb{R}^{k}} \\
& +\rho M \int_{t}^{\tau} e^{\beta(t-r)}\left[\left\|\mathscr{S}_{\varepsilon}\left(v^{\varepsilon}\right)-\tilde{\mathscr{S}}_{\varepsilon}\left(\tilde{v}^{\varepsilon}\right)\right\|_{X_{\varepsilon}^{\frac{1}{2}}}+\left\|v^{\varepsilon}-\tilde{v}^{\varepsilon}\right\|_{\mathbb{R}^{k}}\right] d r \\
\leqslant & M e^{\beta(t-\tau)}\|\eta-\tilde{\eta}\|_{\mathbb{R}^{k}} \\
& +\rho M \int_{t}^{\tau} e^{\beta(t-r)}\left[\left\|\mathscr{S}_{\varepsilon}\left(\tilde{v}^{\varepsilon}\right)-\tilde{\mathscr{S}}_{\varepsilon}\left(\tilde{v}^{\varepsilon}\right)\right\|_{X_{\varepsilon}^{\frac{1}{2}}}+(1+\Delta)\left\|v^{\varepsilon}-\tilde{v}^{\varepsilon}\right\|_{\mathbb{R}^{k}}\right] d r \\
\leqslant & M e^{\beta(t-\tau)}\|\eta-\tilde{\eta}\|_{\mathbb{R}^{k}} \\
& +\rho M \int_{t}^{\tau} e^{\beta(t-r)}\left[(1+\Delta)\left\|v^{\varepsilon}-\tilde{v}^{\varepsilon}\right\|_{\mathbb{R}^{k}}+\left\|\mathscr{S}_{\varepsilon}-\tilde{\mathscr{S}}_{\varepsilon}\right\|\right] d r \\
\leqslant & M e^{\beta(t-\tau)}\|\eta-\tilde{\eta}\|_{\mathbb{R}^{k}} \\
& +\rho M(1+\Delta) \int_{t}^{\tau} e^{\beta(t-r)}\left\|v^{\varepsilon}-\tilde{v}^{\varepsilon}\right\|_{\mathbb{R}^{k}} d r \\
& +\rho M\left\|\mathscr{S}_{\varepsilon}-\tilde{\mathscr{S}}_{\varepsilon}\right\| \int_{t}^{\tau} e^{\beta(t-r)} d r .
\end{aligned}
$$

Seja $\phi(t)=e^{-\beta(t-\tau)}\left\|v^{\varepsilon}(t)-\tilde{v}^{\varepsilon}(t)\right\|_{\mathbb{R}^{k}}$. Então

$$
\phi(t) \leqslant M\|\eta-\tilde{\eta}\|_{\mathbb{R}^{k}}+\rho M \int_{t}^{\tau} e^{\beta(\tau-r)} d r\left\|\mathscr{S}_{\varepsilon}-\tilde{\mathscr{S}}_{\varepsilon}\right\|+\rho M(1+\Delta) \int_{t}^{\tau} \phi(r) d r .
$$

Assim, resulta da Desigualdade de Gronwall Generalizada que

$$
\begin{aligned}
\left\|v^{\varepsilon}(t)-\tilde{v}^{\varepsilon}(t)\right\|_{\mathbb{R}^{k}} \leqslant & {\left[M e^{\beta(t-\tau)}\|\eta-\tilde{\eta}\|_{\mathbb{R}^{k}}+\rho M \beta^{-1}\left(1-e^{\beta(t-\tau)}\right)\left\|\mathscr{S}_{\varepsilon}-\tilde{\mathscr{S}}_{\varepsilon}\right\|\right] } \\
& \times e^{[\rho M(1+\Delta)-\beta](\tau-t)} \\
\leqslant & {\left[M\|\eta-\tilde{\eta}\|_{\mathbb{R}^{k}}+\rho M \beta^{-1}\left\|\mathscr{S}_{\varepsilon}-\tilde{\mathscr{S}}_{\varepsilon}\right\|\right] e^{[\rho M(1+\Delta)-\beta](\tau-t)} }
\end{aligned}
$$


Agora, como

$$
\begin{aligned}
\left\|\Phi\left(\mathscr{S}_{\varepsilon}\right)(\eta)-\Phi\left(\tilde{\mathscr{S}}_{\varepsilon}\right)(\tilde{\eta})\right\|_{X_{\varepsilon}^{\frac{1}{2}} \leqslant} & M \int_{-\infty}^{\tau}(\tau-r)^{-\left(\frac{1+s}{2}\right)} e^{-\beta(\tau-r)} \\
\times & \left\|G_{\mathcal{\varepsilon}}\left(v^{\varepsilon}, \mathscr{S}_{\varepsilon}\left(v^{\varepsilon}\right)\right)-G_{\varepsilon}\left(\tilde{v}_{\varepsilon}, \tilde{\mathscr{S}}_{\varepsilon}\left(\tilde{v}^{\varepsilon}\right)\right)\right\|_{X_{\varepsilon}^{-\frac{s}{2}}} d r \\
\leqslant & \rho M \int_{-\infty}^{\tau}(\tau-r)^{-\left(\frac{1+s}{2}\right)} e^{-\beta(\tau-r)}\left[(1+\Delta)\left\|v^{\varepsilon}-\tilde{v}^{\varepsilon}\right\|_{\mathbb{R}^{k}}\right. \\
& \left.+\left\|\mathscr{S}_{\varepsilon}-\tilde{\mathscr{S}}_{\varepsilon}\right\|\right] d r
\end{aligned}
$$

substituindo as estimativas obtidas para $\left\|v^{\varepsilon}-\tilde{v}^{\varepsilon}\right\|_{\mathbb{R}^{k}}$, obtemos

$$
\begin{aligned}
\left\|\Phi\left(\mathscr{S}_{\varepsilon}\right)(\eta)-\Phi\left(\tilde{\mathscr{S}}_{\varepsilon}\right)(\tilde{\eta})\right\|_{X_{\varepsilon}^{\frac{1}{2}} \leqslant} & \rho M \beta^{\left(\frac{s-1}{2}\right)} \Gamma\left(\frac{1-s}{2}\right)\left[1+\frac{\rho M(1+\Delta)}{2 \beta-\rho M(1+\Delta)}\right]\left\|\mathscr{S}_{\varepsilon}-\tilde{\mathscr{S}}_{\varepsilon}\right\| \\
& +\rho M^{2}(1+\Delta)\left(\frac{\beta}{2}\right)^{\left(\frac{s-1}{2}\right)} \Gamma\left(\frac{1-s}{2}\right)\|\eta-\tilde{\eta}\|_{\mathbb{R}^{k}} .
\end{aligned}
$$

Sejam

$$
I_{\mathscr{S}}(\varepsilon):=\rho M \beta^{\left(\frac{s-1}{2}\right)} \Gamma\left(\frac{1-s}{2}\right)\left[1+\frac{\rho M(1+\Delta)}{2 \beta-\rho M(1+\Delta)}\right]
$$

$\mathrm{e}$

$$
I_{\eta}(\varepsilon):=\rho M^{2}(1+\Delta)\left(\frac{\beta}{2}\right)^{\left(\frac{s-1}{2}\right)} \Gamma\left(\frac{1-s}{2}\right) .
$$

Dado $\vartheta<1$, existe um $\rho_{0}$ tal que, para $\rho \leqslant \rho_{0}, I_{\mathscr{S}}(\varepsilon) \leqslant \vartheta$ e $I_{\eta}(\varepsilon) \leqslant \Delta$ e

$$
\left\|\Phi\left(\mathscr{S}_{\varepsilon}\right)(\eta)-\Phi\left(\tilde{\mathscr{S}}_{\varepsilon}\right)(\tilde{\eta})\right\|_{X_{\varepsilon}^{\frac{1}{2}}} \leqslant \Delta\|\eta-\tilde{\eta}\|_{\mathbb{R}^{k}}+\vartheta\left\|\mathscr{S}_{\varepsilon}-\tilde{\mathscr{S}}_{\varepsilon}\right\|
$$

Das desigualdades (4.25) e (4.26), segue que $\Phi$ é uma contração de $\mathscr{X}$ em $\mathscr{X}$. Logo, existe um único ponto fixo $\mathscr{S}_{\varepsilon}^{*}=\Phi\left(\mathscr{S}_{\varepsilon}^{*}\right)$ em $\mathscr{X}$.

Provemos agora que $\mathrm{W}_{\varepsilon}^{u}=\left\{\left(v, \mathscr{S}_{\varepsilon}^{*}(v)\right): v \in \mathbb{R}^{k}\right\}$ é uma variedade invariante para (4.21). Seja $\left(v^{0}, z^{0}\right) \in \mathrm{W}_{\varepsilon}^{u}, z^{0}=\mathscr{S}_{\varepsilon}^{*}\left(v^{0}\right)$. Denotemos por $v_{*}^{\varepsilon}(t)$ a solução do problema de valor inicial

$$
\left\{\begin{array}{l}
\dot{v}^{\varepsilon}+\bar{A}_{\varepsilon}^{+} v^{\varepsilon}=H_{\mathcal{\varepsilon}}\left(v^{\varepsilon}, \mathscr{S}_{\varepsilon}^{*}\left(v^{\varepsilon}\right)\right) \\
v^{\varepsilon}(0)=v_{0}^{\varepsilon}
\end{array}\right.
$$

Isto define uma curva $\left(v_{*}^{\varepsilon}(t), \mathscr{S}_{\varepsilon}^{*}\left(v_{*}^{\varepsilon}(t)\right)\right) \in \mathrm{W}_{\varepsilon}^{u}, t \in \mathbb{R}$. Contudo, a única solução da equação

$$
\dot{z}^{\varepsilon}+\bar{A}_{\varepsilon}^{-} z^{\varepsilon}=G_{\varepsilon}\left(v_{*}^{\varepsilon}(t), \mathscr{S}_{\varepsilon}^{*}\left(v_{*}^{\varepsilon}(t)\right)\right)
$$

que permanece limitada quando $t \rightarrow-\infty$ é

$$
z_{*}^{\varepsilon}(t)=\int_{-\infty}^{t} e^{-\bar{A}_{\varepsilon}^{-}(t-r)} G_{\varepsilon}\left(v_{*}^{\varepsilon}(r), \mathscr{S}_{\varepsilon}^{*}\left(v_{*}^{\varepsilon}(r)\right) d r=\mathscr{S}_{\varepsilon}^{*}\left(v_{*}^{\varepsilon}(t)\right)\right.
$$


Portanto, $\left(v_{*}^{\varepsilon}(t), \mathscr{S}_{\varepsilon}^{*}\left(v_{*}^{\varepsilon}(t)\right)\right)$ é uma solução de (4.21) passando por $\left(v^{0}, z^{0}\right)$, o que prova a invariância.

A seguir provamos que se $H_{\varepsilon}$ e $G_{\varepsilon}$ satisfazem (4.20) para todo $\left(v^{\varepsilon}, z^{\varepsilon}\right) \in X_{\varepsilon}^{\frac{1}{2}} \times\left(I-Q_{\varepsilon}^{+}\right) X_{\mathcal{\varepsilon}}^{\frac{1}{2}}$ com $\rho$ apropriadamente pequeno e se $\left(v^{\varepsilon}(t), z^{\varepsilon}(t)\right), t \in \mathbb{R}$ é uma solução global para (4.17) que está em $W^{u}\left(u_{\mathcal{\varepsilon}}^{*}\right)$, então $z^{\varepsilon}(t)=\mathscr{S}_{\varepsilon}^{*}\left(v^{\varepsilon}(t)\right)$, para todo $t \in \mathbb{R}$. Nosso objetivo é mostrar que existem constantes $M \geqslant 1$ e $\gamma>0$ de tal maneira que

$$
\left\|z^{\varepsilon}(t)-\mathscr{S}_{\varepsilon}^{*}\left(v^{\varepsilon}(t)\right)\right\|_{X_{\varepsilon}^{-\frac{s}{2}}} \leqslant M e^{-\gamma\left(t-t_{0}\right)}\left\|z^{\varepsilon}\left(t_{0}\right)-\mathscr{S}_{\varepsilon}^{*}\left(v^{\varepsilon}\left(t_{0}\right)\right)\right\|_{X_{\varepsilon}^{-\frac{s}{2}}}, \quad t_{0} \leqslant t .
$$

Quando $t_{0} \rightarrow-\infty$, obtemos $z^{\varepsilon}(t)=\mathscr{S}_{\varepsilon}^{*}\left(v^{\varepsilon}(t)\right)$ para todo $t \in \mathbb{R}$.

Sejam $\xi^{\varepsilon}(t)=z^{\varepsilon}(t)-\mathscr{S}_{\varepsilon}^{*}\left(v^{\varepsilon}(t)\right)$ e $y^{\varepsilon}(r, t), r \leqslant t$ a solução de

$$
\left\{\begin{array}{l}
\dot{y}^{\varepsilon}+\bar{A}_{\varepsilon}^{+} y^{\varepsilon}=H_{\varepsilon}\left(y^{\varepsilon}, \mathscr{S}_{\varepsilon}^{*}\left(y^{\varepsilon}\right)\right), \quad r \leqslant t \\
y^{\varepsilon}(t, t)=v^{\varepsilon}(t) .
\end{array}\right.
$$

Assim,

$$
\begin{aligned}
\| y^{\varepsilon}(r, t) & -v^{\varepsilon}(r)\left\|_{\mathbb{R}^{k}}=\right\| \int_{t}^{r} e^{-\bar{A}_{\varepsilon}^{+}(r-\theta)}\left[H_{\mathcal{E}}\left(y^{\varepsilon}(\theta, t), \mathscr{S}_{\varepsilon}^{*}\left(y^{\varepsilon}(\theta, t)\right)\right)-H_{\mathcal{\varepsilon}}\left(v^{\varepsilon}(\theta), z^{\varepsilon}(\theta)\right)\right] d \theta \|_{\mathbb{R}^{k}} \\
& \leqslant \rho M \int_{r}^{t} e^{\beta(r-\theta)}\left[(1+\Delta)\left\|y^{\varepsilon}(\theta, t)-v^{\varepsilon}(\theta)\right\|_{\mathbb{R}^{k}}+\left\|\mathscr{S}_{\varepsilon}^{*}\left(v^{\varepsilon}(\theta)\right)-z^{\varepsilon}(\theta)\right\|_{X_{\varepsilon}^{-\frac{s}{2}}}\right] d \theta \\
& \leqslant \rho M \int_{r}^{t} e^{\beta(r-\theta)}\left[(1+\Delta)\left\|y^{\varepsilon}(\theta, t)-v^{\varepsilon}(\theta)\right\|_{\mathbb{R}^{k}}+\left\|\xi^{\varepsilon}(\theta)\right\|_{X_{\varepsilon}^{-\frac{s}{2}}}\right] d \theta .
\end{aligned}
$$

$\operatorname{Se} \phi^{\varepsilon}(r)=e^{-\beta r}\left\|y^{\varepsilon}(r, t)-v^{\varepsilon}(r)\right\|_{\mathbb{R}^{k}}$, então

$$
\phi^{\varepsilon}(r) \leqslant \rho M(1+\Delta) \int_{r}^{t} \phi^{\varepsilon}(\theta) d \theta+\rho M \int_{r}^{t} e^{-\beta \theta}\left\|\xi^{\varepsilon}(\theta)\right\|_{X_{\varepsilon}^{-\frac{s}{2}}} d \theta, \quad r \leqslant t .
$$

Da Desigualdade de Gronwall segue que

$$
\left\|y^{\varepsilon}(r, t)-v^{\varepsilon}(r)\right\|_{\mathbb{R}^{k}} \leqslant \rho M \int_{r}^{t} e^{-(\beta-\rho M(1+\Delta))(\theta-r)}\left\|\xi^{\varepsilon}(\theta)\right\|_{X_{\varepsilon}^{-\frac{s}{2}}} d \theta, \quad r \leqslant t .
$$

Seja agora $r \leqslant t_{0} \leqslant t$. Então,

$$
\begin{aligned}
\| y^{\varepsilon}(r, t) & -y^{\varepsilon}\left(r, t_{0}\right)\left\|_{\mathbb{R}^{k}}=\right\| e^{-\bar{A}_{\varepsilon}^{+}\left(r-t_{0}\right)}\left[y^{\varepsilon}\left(t_{0}, t\right)-v\left(t_{0}\right)\right] \|_{\mathbb{R}^{k}} \\
& +\left\|\int_{t_{0}}^{r} e^{-\bar{A}_{\varepsilon}^{+}(r-\theta)}\left[H_{\mathcal{\varepsilon}}\left(y^{\varepsilon}(\theta, t), \mathscr{S}_{\varepsilon}^{*}\left(y^{\varepsilon}(\theta, t)\right)\right)-H_{\mathcal{\varepsilon}}\left(y^{\varepsilon}\left(\theta, t_{0}\right), \mathscr{S}_{\varepsilon}^{*}\left(y^{\varepsilon}\left(\theta, t_{0}\right)\right)\right)\right] d \theta\right\|_{\mathbb{R}^{k}} \\
& \leqslant \rho M^{2} e^{\beta\left(r-t_{0}\right)} \int_{t_{0}}^{t} e^{-(\beta-\rho M(1+\Delta))\left(\theta-t_{0}\right)}\left\|\xi^{\varepsilon}(\theta)\right\|_{X_{\varepsilon}^{-\frac{s}{2}}} d \theta \\
& +\rho M \int_{r}^{t_{0}} e^{\beta(r-\theta)}(1+\Delta)\left\|y^{\varepsilon}(\theta, t)-y^{\varepsilon}\left(\theta, t_{0}\right)\right\|_{\mathbb{R}^{k}} d \theta
\end{aligned}
$$


Usando novamente a Desigualdade de Gronwall, obtemos

$$
\left\|y^{\varepsilon}(r, t)-y^{\varepsilon}\left(r, t_{0}\right)\right\|_{\mathbb{R}^{k}} \leqslant \rho M^{2} \int_{t_{0}}^{t} e^{-(\beta-\rho M(1+\Delta))(\theta-r)}\left\|\xi^{\varepsilon}(\theta)\right\|_{X_{\mathcal{\varepsilon}}^{-\frac{s}{2}}} d \theta
$$

Vamos agora estimar $\xi(t)$. Notemos que

$$
\begin{aligned}
\xi^{\varepsilon}(t) & -e^{-\bar{A}_{\varepsilon}^{-}\left(t-t_{0}\right)} \xi\left(t_{0}\right)=z^{\varepsilon}(t)-\mathscr{S}_{\varepsilon}^{*}\left(v^{\varepsilon}(t)\right)-e^{-\bar{A}_{\varepsilon}^{-}\left(t-t_{0}\right)}\left[z^{\varepsilon}\left(t_{0}\right)-\mathscr{S}_{\varepsilon}^{*}\left(v^{\varepsilon}\left(t_{0}\right)\right)\right] \\
& =\int_{t_{0}}^{t} e^{-\bar{A}_{\varepsilon}^{-}(t-r)} G_{\varepsilon}\left(v^{\varepsilon}(r), z^{\varepsilon}(r)\right) d r-\mathscr{S}_{\varepsilon}^{*}\left(v^{\varepsilon}(t)\right)+e^{-\bar{A}_{\varepsilon}^{-}\left(t-t_{0}\right)} \mathscr{S}_{\varepsilon}^{*}\left(v^{\varepsilon}\left(t_{0}\right)\right) \\
& =\int_{t_{0}}^{t} e^{-\bar{A}_{\bar{\varepsilon}}^{-}(t-r)} G_{\varepsilon}\left(v^{\varepsilon}(r), z^{\varepsilon}(r)\right) d r-\int_{-\infty}^{t} e^{-\bar{A}_{\varepsilon}^{-}(t-r)} G_{\varepsilon}\left(y^{\varepsilon}(r, t), \mathscr{S}_{\varepsilon}^{*}\left(y^{\varepsilon}(r, t)\right)\right) d r \\
& +e^{-\bar{A}_{\varepsilon}^{-}\left(t-t_{0}\right)} \int_{-\infty}^{t_{0}} e^{-\bar{A}_{\varepsilon}^{-}\left(t_{0}-r\right)} G_{\varepsilon}\left(y^{\varepsilon}\left(r, t_{0}\right), \mathscr{S}_{\varepsilon}^{*}\left(y^{\varepsilon}\left(r, t_{0}\right)\right)\right) d r \\
& =\int_{t_{0}}^{t} e^{-\bar{A}_{\bar{\varepsilon}}^{-}(t-r)}\left[G_{\varepsilon}\left(v^{\varepsilon}(r), z^{\varepsilon}(r)\right)-G_{\varepsilon}\left(y^{\varepsilon}(r, t), \mathscr{S}_{\varepsilon}^{*}\left(y^{\varepsilon}(r, t)\right)\right)\right] d r \\
& -\int_{-\infty}^{t_{0}} e^{-\bar{A}_{\varepsilon}^{-}(t-r)}\left[G_{\varepsilon}\left(y^{\varepsilon}(r, t), \mathscr{S}_{\varepsilon}^{*}\left(y^{\varepsilon}(r, t)\right)\right)-G_{\varepsilon}\left(y^{\varepsilon}\left(r, t_{0}\right), \mathscr{S}_{\varepsilon}^{*}\left(y^{\varepsilon}\left(r, t_{0}\right)\right)\right)\right] d r
\end{aligned}
$$

Assim, usando (4.28) e (4.29),

$$
\begin{aligned}
& \left\|\xi^{\varepsilon}(t)-e^{-\bar{A}_{\varepsilon}^{-}\left(t-t_{0}\right)} \xi^{\varepsilon}\left(t_{0}\right)\right\|_{X_{\varepsilon}^{-\frac{s}{2}}} \\
& \leqslant \rho M \int_{t_{0}}^{t} e^{-\beta(t-r)}\left[\left\|v^{\varepsilon}(r)-y^{\varepsilon}(r, t)\right\|_{\mathbb{R}^{k}}+\left\|z^{\varepsilon}(r)-\mathscr{S}_{\varepsilon}^{*}\left(y^{\varepsilon}(r, t)\right)\right\|_{\mathbb{R}^{k}}\right] d r \\
& +\rho M(1+\Delta) \int_{-\infty}^{t_{0}} e^{-\beta(t-s)}\left\|y^{\varepsilon}(r, t)-y^{\varepsilon}\left(r, t_{0}\right)\right\|_{\mathbb{R}^{k}} d r \\
& \leqslant \rho M \int_{t_{0}}^{t} e^{-\beta(t-r)}\left\|\xi^{\varepsilon}(r)\right\|_{X_{\varepsilon}^{-\frac{s}{2}}} d r+\rho M(1+\Delta) \int_{t_{0}}^{t} e^{-\beta(t-r)}\left\|v^{\varepsilon}(r)-y^{\varepsilon}(r, t)\right\|_{\mathbb{R}^{k}} d r \\
& +\rho M(1+\Delta) \int_{-\infty}^{t_{0}} e^{-\beta(t-r)}\left\|y^{\varepsilon}(r, t)-y^{\varepsilon}\left(r, t_{0}\right)\right\|_{\mathbb{R}^{k}} d r \\
& \leqslant \rho M \int_{t_{0}}^{t} e^{-\beta(t-r)}\left\|\xi^{\varepsilon}(r)\right\|_{X_{\varepsilon}^{-\frac{s}{2}}} d r \\
& +\rho^{2} M^{2}(1+\Delta) \int_{t_{0}}^{t} e^{-\beta(t-r)} \int_{r}^{t} e^{-(\beta-\rho M(1+\Delta))(\theta-r)}\left\|\xi^{\varepsilon}(\theta)\right\|_{X_{\mathcal{\varepsilon}}^{-\frac{s}{2}}} d \theta d r \\
& +\rho^{2} M^{3}(1+\Delta) \int_{-\infty}^{t_{0}} e^{-\beta(t-s)} \int_{t_{0}}^{t} e^{-(\beta-\rho M(1+\Delta))(\theta-r)}\left\|\xi^{\varepsilon}(\theta)\right\|_{X_{\varepsilon}^{-\frac{s}{2}}} d \theta d r \\
& \leqslant \rho M \int_{t_{0}}^{t} e^{-\beta(t-r)}\left\|\xi^{\varepsilon}(r)\right\|_{X_{\varepsilon}^{-\frac{s}{2}}} d r \\
& +\rho^{2} M^{2}(1+\Delta) e^{-\beta t} \int_{t_{0}}^{t} e^{-(\beta-\rho M(1+\Delta)) \theta}\left\|\xi^{\varepsilon}(\theta)\right\|_{X_{\varepsilon}^{-\frac{s}{2}}} \int_{t_{0}}^{\theta} e^{(2 \beta-\rho M(1+\Delta)) r} d r d \theta \\
& +\rho^{2} M^{3}(1+\Delta) e^{-\beta t} \int_{t_{0}}^{t} e^{-(\beta-\rho M(1+\Delta)) \theta}\left\|\xi^{\varepsilon}(\theta)\right\|_{X_{\varepsilon}^{-\frac{s}{2}}} \int_{-\infty}^{t_{0}} e^{(2 \beta-\rho M(1+\Delta)) r} d r d \theta
\end{aligned}
$$


de maneira que

$$
\begin{aligned}
\| \xi^{\varepsilon}(t) & -e^{-\bar{A}_{\varepsilon}^{-}\left(t-t_{0}\right)} \xi^{\varepsilon}\left(t_{0}\right)\left\|_{X_{\varepsilon}^{-\frac{s}{2}}} \leqslant\left[\rho M+\frac{\rho^{2} M^{2}(1+\Delta)}{2 \beta-\rho M(1+\Delta)}\right] \int_{t_{0}}^{t} e^{-\beta(t-r)}\right\| \xi^{\varepsilon}(r) \|_{X_{\varepsilon}^{-\frac{s}{2}}} d r \\
& +\frac{\rho^{2} M^{3}(1+\Delta)}{2 \beta-\rho M(1+\Delta)} e^{-\beta\left(t-t_{0}\right)} \int_{t_{0}}^{t} e^{-(\beta-\rho M(1+\Delta))\left(\theta-t_{0}\right)}\left\|\xi^{\varepsilon}(\theta)\right\|_{X_{\varepsilon}^{-\frac{s}{2}}} d \theta
\end{aligned}
$$

e portanto,

$$
\begin{aligned}
e^{\beta\left(t-t_{0}\right)}\left\|\xi^{\varepsilon}(t)\right\|_{X_{\varepsilon}^{-\frac{s}{2}}} \leqslant M\left\|\xi^{\varepsilon}\left(t_{0}\right)\right\|_{X_{\varepsilon}^{-\frac{s}{2}}}+\left[\rho M+\frac{\rho^{2} M^{2}(1+\Delta)}{2 \beta-\rho M(1+\Delta)}\right] \int_{t_{0}}^{t} e^{\beta\left(r-t_{0}\right)}\left\|\xi^{\varepsilon}(r)\right\|_{X_{\varepsilon}^{-\frac{s}{2}}} d r \\
+\frac{\rho^{2} M^{3}(1+\Delta)}{2 \beta-\rho M(1+\Delta)} \int_{t_{0}}^{t} e^{-(2 \beta-\rho M(1+\Delta))\left(r-t_{0}\right)} e^{\beta\left(r-t_{0}\right)}\left\|\xi^{\varepsilon}(r)\right\|_{X_{\varepsilon}^{-\frac{s}{2}}} d r \\
\leqslant M\left\|\xi^{\varepsilon}\left(t_{0}\right)\right\|_{X_{\varepsilon}^{-\frac{s}{2}}}+\left[\rho M+\frac{\rho^{2} M^{2}(1+\Delta)(1+M)}{2 \beta-\rho M(1+\Delta)}\right] \int_{t_{0}}^{t} e^{\beta\left(r-t_{0}\right)}\left\|\xi^{\varepsilon}(r)\right\|_{X_{\varepsilon}^{-\frac{s}{2}}} d r .
\end{aligned}
$$

Da Desigualdade de Gronwall segue ainda que

$$
\left\|\xi^{\varepsilon}(t)\right\|_{X_{\varepsilon}^{-\frac{s}{2}}} \leqslant M\left\|\xi^{\varepsilon}\left(t_{0}\right)\right\|_{X_{\varepsilon}^{-\frac{s}{2}}} e^{-\gamma\left(t-t_{0}\right)}
$$

onde

$$
\gamma=\beta-\left[\rho M+\frac{\rho^{2} M^{2}(1+\Delta)(1+M)}{2 \beta-\rho M(1+\Delta)}\right] .
$$

Logo, $z^{\varepsilon}(t)=\mathscr{S}_{\varepsilon}^{*}\left(v^{\varepsilon}(t)\right)$, para todo $t \in \mathbb{R}$.

Mostramos, por fim, que os pontos fixos $\mathscr{S}_{\varepsilon}^{*}$ dependem continuamente de $\varepsilon$ em $\varepsilon=0$, ou seja, se $0<\varepsilon \leqslant \bar{\varepsilon}$ é tal que a variedade instável é dada pelo gráfico de $\mathscr{S}_{\varepsilon}^{*}, 0 \leqslant \varepsilon \leqslant \bar{\varepsilon}$, queremos mostrar que

$$
\|\| \mathscr{S}_{\varepsilon}^{*}-\mathscr{S}_{0}^{*}\left|\left\|\mid:=\sup _{\eta \in B_{\mathbb{R}}^{k}(0, R)}\right\| \mathscr{S}_{\varepsilon}^{*}(\eta)-\mathscr{S}_{0}^{*}(\eta) \|_{X_{\varepsilon}^{\frac{1}{2}}} \rightarrow 0 \text { quando } \varepsilon \rightarrow 0 .\right.
$$

Para tanto, observemos primeiramente que, como a aplicação $\mathbb{R}^{k} \ni v^{0} \mapsto G_{0}\left(v^{0}, \mathscr{S}_{0}^{*}\left(v^{0}\right)\right)$ é contínua, ela leva subconjuntos limitados de $\mathbb{R}^{k}$ em subconjuntos relativamente compactos de $X_{0}^{-\frac{s}{2}}$.

Portanto,

$$
\begin{aligned}
\left\|\mathscr{S}_{\varepsilon}^{*}(\eta)-\mathscr{S}_{0}^{*}(\eta)\right\|_{X_{\varepsilon}^{\frac{1}{2}} \leqslant} & \int_{-\infty}^{\tau}\left\|e^{-\bar{A}_{\bar{\varepsilon}}^{-}(\tau-r)} G_{\varepsilon}\left(v^{\varepsilon}, \mathscr{S}_{\varepsilon}^{*}\left(v^{\varepsilon}\right)\right)-e^{-\bar{A}_{0}^{-}(\tau-r)} G_{0}\left(v^{0}, \mathscr{S}_{0}^{*}\left(v^{0}\right)\right)\right\|_{X_{\varepsilon}^{\frac{1}{2}}} d r \\
\leqslant & \int_{-\infty}^{\tau}\left\|e^{-\bar{A}_{\bar{\varepsilon}}^{-}(\tau-r)} G_{\varepsilon}\left(v^{\varepsilon}, \mathscr{S}_{\varepsilon}^{*}\left(v^{\varepsilon}\right)\right)-e^{-\bar{A}_{\varepsilon}^{-}(\tau-r)} G_{\varepsilon}\left(v^{0}, \mathscr{S}_{0}^{*}\left(v^{0}\right)\right)\right\|_{X_{\varepsilon}^{\frac{1}{2}}} d r \\
& +\int_{-\infty}^{\tau}\left\|e^{-\bar{A}_{\varepsilon}^{-}(\tau-r)} G_{\varepsilon}\left(v^{0}, \mathscr{S}_{0}^{*}\left(v^{0}\right)\right)-e^{-\bar{A}_{\varepsilon}^{-}(\tau-r)} G_{0}\left(v^{0}, \mathscr{S}_{0}^{*}\left(v^{0}\right)\right)\right\|_{X_{\varepsilon}^{\frac{1}{2}}} d r \\
& +\int_{-\infty}^{\tau}\left\|e^{-\bar{A}_{\varepsilon}^{-}(\tau-r)} G_{0}\left(v^{0}, \mathscr{S}_{0}^{*}\left(v^{0}\right)\right)-e^{-\bar{A}_{0}^{-}(\tau-r)} G_{0}\left(v^{0}, \mathscr{S}_{0}^{*}\left(v^{0}\right)\right)\right\|_{X_{\varepsilon}^{\frac{1}{2}}} d r .
\end{aligned}
$$


Denotando as três últimas integrais por $I_{1}, I_{2}$ e $I_{3}$, respectivamente, temos:

$$
\begin{aligned}
I_{1} \leqslant & \int_{-\infty}^{\tau} M e^{-\beta(\tau-r)}(\tau-r)^{-\left(\frac{1+s}{2}\right)} \rho\left[(1+\Delta)\left\|v^{\varepsilon}-v^{0}\right\|_{\mathbb{R}^{k}}+\mid\left\|\mathscr{S}_{\varepsilon}^{*}-\mathscr{S}_{0}^{*}\right\| \|\right] d r \\
\leqslant & \rho M \beta^{\left(\frac{s-1}{2}\right)} \Gamma\left(\frac{1-s}{2}\right)\|\| \mathscr{S}_{\varepsilon}^{*}-\mathscr{S}_{0}^{*} \mid\|\| \\
& +\rho M(1+\Delta) \int_{-\infty}^{\tau} e^{-\beta(\tau-r)}(\tau-r)^{-\left(\frac{1+s}{2}\right)}\left\|v^{\varepsilon}(r)-v^{0}(r)\right\|_{\mathbb{R}^{k}} d r
\end{aligned}
$$

Como $G_{\varepsilon} \stackrel{\varepsilon \rightarrow 0}{\longrightarrow} G_{0}$ pontualmente sobre compactos, temos que $G_{\varepsilon} \stackrel{\varepsilon \rightarrow 0}{\longrightarrow} G_{0}$ uniformemente e portanto $I_{2}$ é $o(1)$ quando $\varepsilon \rightarrow 0$. Ainda, como $G_{0}\left(v^{0}, \mathscr{S}_{0}^{*}\left(v^{0}\right)\right)$ está em um conjunto compacto de $X_{0}^{-\frac{s}{2}}$, garantimos, como no Teorema 3.10 , que $I_{3}$ também é $o(1)$ quando $\varepsilon \rightarrow 0$. Logo,

$$
\begin{aligned}
\left\|\mathscr{S}_{\varepsilon}^{*}(\eta)-\mathscr{S}_{0}^{*}(\eta)\right\|_{X_{\varepsilon}^{\frac{1}{2}} \leqslant} & o(1)+\rho M \beta^{\left(\frac{s-1}{2}\right)} \Gamma\left(\frac{1-s}{2}\right)\|\| \mathscr{S}_{\varepsilon}^{*}-\mathscr{S}_{0}^{*}\|\| \\
& +\rho M(1+\Delta) \int_{-\infty}^{\tau} e^{-\beta(\tau-r)}(\tau-r)^{\left(\frac{s-1}{2}\right)}\left\|v^{\varepsilon}(r)-v^{0}(r)\right\|_{\mathbb{R}^{k}} d r .
\end{aligned}
$$

É suficiente então estimarmos $\left\|v^{\varepsilon}(t)-v^{0}(t)\right\|_{\mathbb{R}^{k}}$. Como $\|\eta\|_{\mathbb{R}^{k}} \leqslant R$, procedendo como acima, obtemos

$$
\begin{aligned}
\left\|v^{\varepsilon}(t)-v^{0}(t)\right\|_{\mathbb{R}^{k}} \leqslant & \left\|e^{-\bar{A}_{\varepsilon}^{+}(t-\tau)} \eta-e^{-\bar{A}_{0}^{+}(t-\tau)} \eta\right\|_{\mathbb{R}^{k}} \\
& +\int_{t}^{\tau}\left\|e^{-\bar{A}_{\varepsilon}^{+}(t-r)} H_{\mathcal{\varepsilon}}\left(v^{\varepsilon}, \mathscr{S}_{\varepsilon}^{*}\left(v^{\varepsilon}\right)\right)-e^{-\bar{A}_{\varepsilon}^{+}(t-r)} H_{\mathcal{\varepsilon}}\left(v^{0}, \mathscr{S}_{0}^{*}\left(v^{0}\right)\right)\right\|_{\mathbb{R}^{k}} d r \\
& +\int_{t}^{\tau}\left\|e^{-\bar{A}_{\varepsilon}^{+}(t-r)} H_{\mathcal{\varepsilon}}\left(v^{0}, \mathscr{S}_{0}^{*}\left(v^{0}\right)\right)-e^{-\bar{A}_{\varepsilon}^{+}(t-r)} H_{0}\left(v^{0}, \mathscr{S}_{0}^{*}\left(v^{0}\right)\right)\right\|_{\mathbb{R}^{k}} d r \\
& +\int_{t}^{\tau}\left\|e^{-\bar{A}_{\varepsilon}^{+}(t-r)} H_{0}\left(v^{0}, \mathscr{S}_{0}^{*}\left(v^{0}\right)\right)-e^{-\bar{A}_{0}^{+}(t-r)} H_{0}\left(v^{0}, \mathscr{S}_{0}^{*}\left(v^{0}\right)\right)\right\|_{\mathbb{R}^{k}} d r \\
\leqslant & o(1)+\rho M \int_{t}^{\tau} e^{\beta(t-r)} d r\|\| \mathscr{S}_{\varepsilon}^{*}-\mathscr{S}_{0}^{*}\|\| \\
& +\rho M(1+\Delta) \int_{t}^{\tau} e^{\beta(t-r)}\left\|v^{\varepsilon}-v^{0}\right\|_{\mathbb{R}^{k}} d r .
\end{aligned}
$$

Seja $\phi(t)=e^{\beta(\tau-t)}\left\|v^{\varepsilon}(t)-v^{0}(t)\right\|_{\mathbb{R}^{k}}$ Então

$$
\phi(t) \leqslant o(1)+\rho M \int_{t}^{\tau} e^{\beta(\tau-r)} d r\|\| \mathscr{S}_{\varepsilon}^{*}-\mathscr{S}_{0}^{*}\|\| \mid+\rho M(1+\Delta) \int_{t}^{\tau} \phi(r) d r
$$

e obtemos

$$
\left\|v^{\varepsilon}(t)-v^{0}(t)\right\|_{\mathbb{R}^{k}} \leqslant\left[o(1)+\rho M \beta^{-1}\|\| \mathscr{S}_{\varepsilon}^{*}-\mathscr{S}_{0}^{*}\|\|\right] e^{[\rho M(1+\Delta)-\beta](\tau-t)} .
$$


Segue então que

$$
\begin{aligned}
\left\|\mathscr{S}_{\varepsilon}^{*}(\eta)-\mathscr{S}_{0}^{*}(\eta)\right\|_{X_{\varepsilon}^{\frac{1}{2}}} \leqslant o(1)+\rho M \beta^{\left(\frac{s-1}{2}\right)} \Gamma\left(\frac{1-s}{2}\right)\left|\left\|\left|\mathscr{S}_{\varepsilon}^{*}-\mathscr{S}_{0}^{*}\right|\right\|\right| \\
+\rho M(1+\Delta) \int_{-\infty}^{\tau} e^{[-2 \beta+\rho M(1+\Delta)](\tau-s)}(\tau-s)^{-\left(\frac{1+s}{2}\right)} d s \\
{\left[o(1)+\rho M \beta^{-1}\left|\left\|\left|\mathscr{S}_{\varepsilon}^{*}-\mathscr{S}_{0}^{*}\right|\right\|\right|\right] } \\
\quad \leqslant o(1)+\rho M \beta^{\left(\frac{s-1}{2}\right)} \Gamma\left(\frac{1-s}{2}\right)\left|\left\|\left|\mathscr{S}_{\varepsilon}^{*}-\mathscr{S}_{0}^{*}\right|\right\|\right| \\
+\left[\rho M(1+\Delta)(2 \beta-\rho M(1+\Delta))^{\left(\frac{s-1}{2}\right)} \Gamma\left(\frac{1-s}{2}\right) \rho M \beta^{-1}\right]\|\| \mathscr{S}_{\varepsilon}^{*}-\mathscr{S}_{0}^{*}|\|| .
\end{aligned}
$$

Assim,

$$
\begin{aligned}
\|\| \mathscr{S}_{\varepsilon}^{*}-\mathscr{S}_{0}^{*}\|\|= & \sup _{\eta \in B_{\mathbb{R}}^{k}(0, R)}\left\|\mathscr{S}_{\varepsilon}^{*}(\eta)-\mathscr{S}_{0}^{*}(\eta)\right\| \leqslant o(1)+\left\{\rho M \beta^{\left(\frac{s-1}{2}\right)} \Gamma\left(\frac{1-s}{2}\right)\right. \\
& \left.+\left[\rho^{2} M^{2} \beta^{-1}(1+\Delta)(2 \beta-\rho M(1+\Delta))^{\left(\frac{s-1}{2}\right)} \Gamma\left(\frac{1-s}{2}\right)\right]\right\}\|\| \mathscr{S}_{\varepsilon}^{*}-\mathscr{S}_{0}^{*}\|\|,
\end{aligned}
$$

e \|\| $\mathscr{S}_{\varepsilon}^{*}-\mathscr{S}_{0}^{*}|\|| \rightarrow 0$ quando $\varepsilon \rightarrow 0$.

Corolário 4.20. Existe $\delta>0$ de tal maneira que

$$
\mathrm{W}_{\varepsilon, \mathrm{loc}}^{u}=\left\{\left(v^{\varepsilon}, z^{\varepsilon}\right) \in \mathbb{R}^{k} \times\left(I-Q_{\varepsilon}^{+}\right) X_{\varepsilon}^{\frac{1}{2}}:\left(v^{\varepsilon}, z^{\varepsilon}\right) \in W_{\varepsilon}^{u},\|v\|_{\mathbb{R}^{k}}+\left\|z^{\varepsilon}\right\|_{X_{\varepsilon}^{\frac{1}{2}}}<\delta\right\}
$$

é dada por

$$
\mathrm{W}_{\varepsilon, \mathrm{loc}}^{u}=\left\{\left(v^{\varepsilon}, \mathscr{S}_{\varepsilon}^{*}\left(v^{\varepsilon}\right)\right): v^{\varepsilon} \in \mathbb{R}^{k}\right\} \cap\left\{\left(v^{\varepsilon}, z^{\varepsilon}\right) \in \mathbb{R}^{k} \times\left(I-Q_{\varepsilon}^{+}\right) X_{\varepsilon}^{\frac{1}{2}}:\left\|v^{\varepsilon}\right\|_{\mathbb{R}^{k}}+\left\|z^{\varepsilon}\right\|_{X_{\varepsilon}^{\frac{1}{2}}}<\delta\right\} .
$$

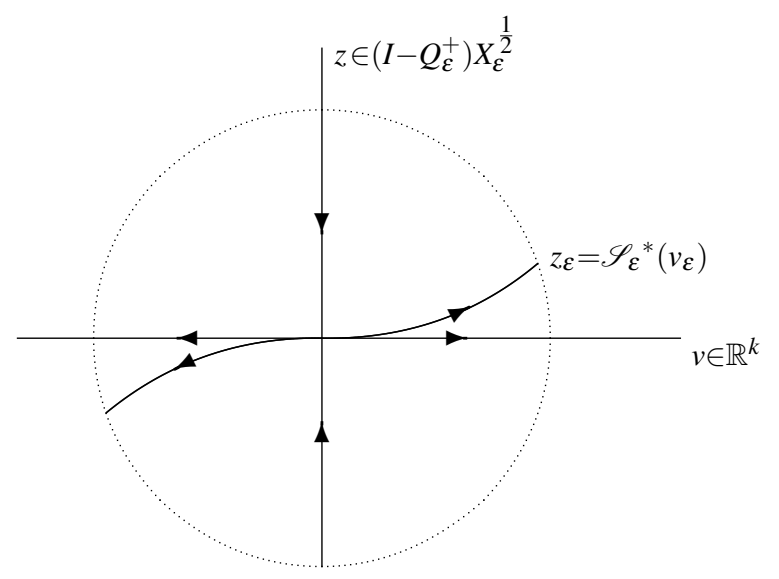

Figura 2: $W_{\varepsilon, \text { loc }}^{u}\left(u_{*}^{\varepsilon}\right)$ 
Como conseqüência imediata da Proposição 4.19, temos

Corolário 4.21. Assumamos que as condições da Proposição 4.19 estejam satisfeitas, que o problema $\left(\mathrm{E}_{0}\right)$ tenha exatamente $n_{0}$ soluções $u_{1}, \ldots, u_{n_{0}}$, e que todas elas sejam hiperbólicas. Então existe $\delta>0$ suficientemente pequeno tal que o problema $\left(\mathrm{E}_{\mathcal{\varepsilon}}\right)$ tem exatamente $n_{0}$ soluções, e suas variedades instáveis locais $\mathrm{W}_{\varepsilon, \text { loc }}^{u}\left(u_{j}^{\varepsilon}\right), j=1, \ldots, n_{0}$, se comportam continuamente em $\varepsilon$ quando $\varepsilon \rightarrow 0$.

\subsection{Semicontinuidade inferior dos atratores}

Para provarmos a semicontinuidade inferior da família de atratores, devemos ainda verificar que os semigrupos gerados pelas equações (2.6) e (2.8) têm estrutura gradiente.

Consideremos, para cada $\varepsilon \in\left(0, \varepsilon_{0}\right]$, o funcional $V_{\varepsilon}: X_{\varepsilon}^{\frac{1}{2}} \rightarrow \mathbb{R}$, dado por

$$
V_{\varepsilon}(\varphi)=\frac{1}{2} \int_{\Omega} p_{\varepsilon}(x)|\nabla \varphi|^{2} d x+\frac{1}{2} \int_{\Omega} \lambda \varphi^{2} d x-\int_{\Omega} H(\varphi) d x,
$$

onde $H$ é uma primitiva de $h$. Este funcional é não-crescente com relação à variável $t$ ao longo das soluções de (2.6). Com efeito, denotemos por conveniência a solução de (2.6) por $u(t, x)$. Então

$$
\frac{d}{d t} V_{\varepsilon}(u)=\int_{\Omega} p_{\varepsilon}(x) \nabla u \nabla u_{t} d x+\int_{\Omega} \lambda u u_{t} d x-\int_{\Omega} h(u) u_{t} d x .
$$

Logo, do Teorema de Gauss, segue que

$$
\begin{aligned}
\frac{d}{d t} V_{\varepsilon}(u) & =\int_{\partial \Omega} p_{\varepsilon}(x) \frac{\partial u}{\partial \vec{n}} u_{t} d x-\int_{\Omega} \operatorname{div}\left(p_{\varepsilon}(x) \nabla u\right) u_{t} d x+\int_{\Omega} \lambda u u_{t} d x-\int_{\Omega} h(u) u_{t} d x \\
& =\int_{\Omega}\left[-\operatorname{div}\left(p_{\varepsilon}(x) \nabla u\right)+\lambda u-h(u)\right] u_{t} d x=\int_{\Omega}-\left(u_{t}\right)^{2} d x \leqslant 0 .
\end{aligned}
$$

Para o problema limite, consideramos o funcional $V_{0}: X_{0}^{\frac{1}{2}} \rightarrow \mathbb{R}$, definido da seguinte forma:

$$
V_{0}(u)=\frac{1}{2} \int_{\Omega_{1}} p_{0}(x)|\nabla u|^{2} d x+\frac{1}{2} \int_{\Omega} \lambda u^{2} d x-\int_{\Omega} H(u) d x .
$$

Teorema 4.22. A família de atratores $\left\{\mathscr{A}_{\varepsilon}: \varepsilon \in\left(0, \varepsilon_{0}\right]\right\}$ é semicontínua inferiormente em $\varepsilon=0$. 
Demonstração. Seja $u \in \mathscr{A}_{0}$. Como $T_{0}(t)$ é gradiente, temos $\mathscr{A}_{0}=\bigcup_{u_{*}^{0} \in \mathscr{E}_{0}} \mathrm{~W}^{u}\left(u 0_{*}\right)$, de modo que $u \in \mathrm{W}^{u}\left(u_{*}\right)$, para algum $u_{*} \in \mathscr{E}_{0}$. Sejam $\tau \in \mathbb{R}$ e $v \in \mathrm{W}_{0, \text { loc }}^{u}\left(u_{*}\right)$ tais que $T_{0}(\tau) v=u$. Seja ainda $u_{*}^{\varepsilon}$ tal que $u_{*}^{\varepsilon} \rightarrow u_{*}$. Da convergência das variedades invariantes segue a existência de uma seqüência $\left\{v^{\varepsilon}\right\}, v^{\varepsilon} \in \mathrm{W}_{\varepsilon}^{u}$,loc $\left(u_{*}^{\varepsilon}\right)$ tal que $v^{\varepsilon} \rightarrow v$ quando $\varepsilon \rightarrow 0$. Finalmente, da continuidade dos semigrupos não-lineares, temos que $T_{\mathcal{E}}(\tau) v^{\varepsilon} \rightarrow T_{0}(\tau) v=u$. Para concluir a demonstração, resta-nos apenas observar que se $u^{\varepsilon}=T_{\varepsilon}(\tau) v^{\varepsilon}$, então $u^{\varepsilon} \in \mathscr{A}_{\varepsilon}$, pois $v^{\varepsilon} \in \bigcup_{u_{*}^{\varepsilon} \in \mathscr{E}_{\varepsilon}} \mathrm{W}^{u}\left(u_{*}^{\varepsilon}\right)=\mathscr{A}_{\varepsilon}$ e $\mathscr{A}_{\varepsilon}$ é invariante.

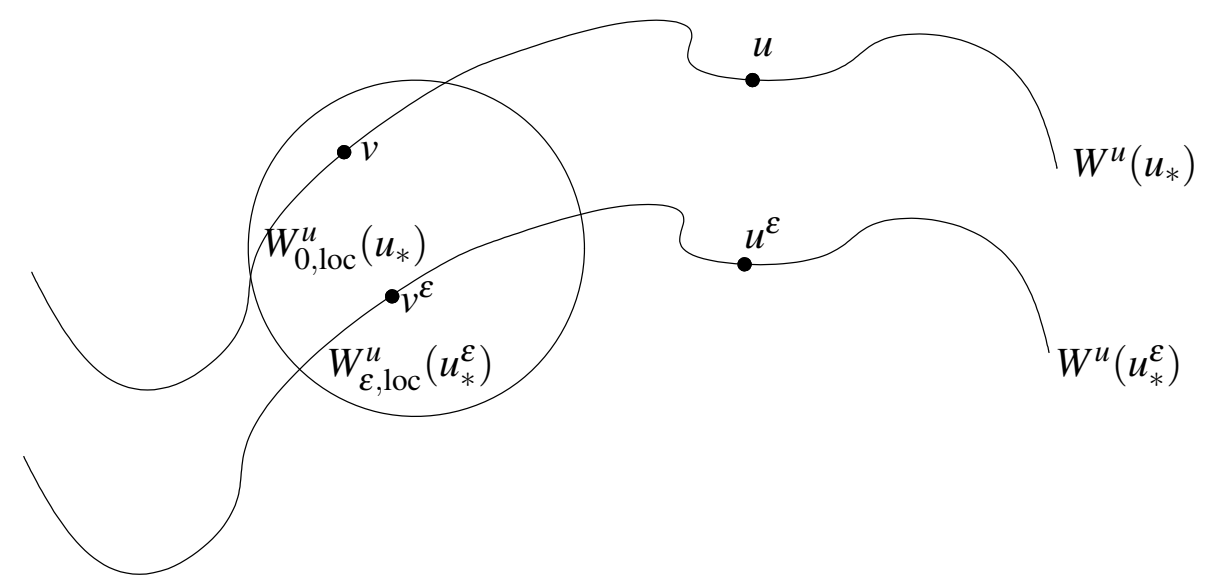

Figura 3: Semicontinuidade inferior dos atratores

Corolário 4.23. A família de atratores $\left\{\mathscr{A}_{\varepsilon}: \varepsilon \in\left(0, \varepsilon_{0}\right]\right\}$ é contínua em $\varepsilon=0$.

Observemos, por fim, que a continuidade da família de atratores $\left\{\mathscr{A}_{\varepsilon}: \varepsilon \in\left(0, \varepsilon_{0}\right]\right\}$ nos fornece uma caracterização para o atrator $\mathscr{A}_{0}$ como um tipo de omega-limite da família $\mathscr{A}_{\varepsilon}$ :

Corolário 4.24. $\mathscr{A}_{0}=\bigcap_{s>0} \overline{\bigcup_{\varepsilon \leqslant s} \mathscr{A}_{S}} \supset \limsup _{\varepsilon \rightarrow 0} \mathscr{A}_{\varepsilon}$. 


\section{Referências bibliográficas}

[1] AdAms, R. A., Sobolev Spaces, Academic Press Inc., (1975).

[2] ARrieta, J. M. And CARvalho, A. N., Spectral convergence and nonlinear dynamics of reaction-diffusion equations under perturbations of the domain, Journal of Differential Equations, 199, 143-178, (2004).

[3] Arrieta, J. M., Carvalho, A. N., Rodríguez-Bernal, A., Attractors for parabolic problems with nonlinear boundary bondition. Uniform bounds, Communications in Partial Differential Equations, 25 (1-2), 1-37, 2000.

[4] Arrieta, J. M., Carvalho, A. N., Rodríguez-Bernal, A., Parabolic problems with nonlinear boundary conditions and critical nonlinearities, Journal of Differential Equations, 156, 376-406, 1999.

[5] Arrieta, J. M., Carvalho, J. M. And Rodríguez-Bernal, A., Upper semicontinuity for attractors of parabolic problems with localized large diffusion and nonlinear boundary conditions, Journal of Differential Equations, 168, 33-59, (2000).

[6] Arrieta, J. M. , Carvalho, A. N. And Rodríguez-Bernal, A., Perturbation of the diffusion and upper semicontinuity for attractors, Journal of Differential Equations, 168, 33-59, (2000).

[7] BrÉzIS, H. Analisis Funcional, teoria e aplicaciones, Masson, Paris, Alianza Editorial, (1983).

[8] Carbone, V. L. And Ruas-Filho, J. G., Continuity of attractors in a singular problem arising in composite materials, Nonlinear Analysis, 65, 1285-1305, (2006).

[9] Carvalho, A. N. And Piskarev, S., A general approximation scheme for attractors of abstract parabolic problems, Notas do ICMC/USP - Série Matematica \# 197, (2005). 
[10] Carvalho, A. N., Oliva, S.M., Pereira, A. L. And Rodríguez-Bernal, A., Attractors for parabolic problems with nonlinear boundary conditions, Journal of Mathematical Anlysis and Applications, 207, 409-461, (1997).

[11] Carvalho, A. N. AND Hines, G., Lower semicontinuity os unstable manifolds for gradient systems, Dynamical Systems and Applications, 9, $\mathrm{n}^{\circ} 1,37-50$, (2000).

[12] Carvalho, A. N., Equações parabólicas semilineares, Notas de Aula, ICMC-USP, (2002).

[13] Conway, E., HofF, D. AND Smoller, J., Large time behavior of solutions of systems of nonlinear reaction-diffusion equations, SIAM J. Appl. Math. 35, 1-16, (1978).

[14] Hale, J. K., Asymptotic behavior of dissipative systems, Mathematics Surveys and Monographs, 25, American Mathematical Society, (1989).

[15] Henry, D. B. , Geometric theory of semilinear parabolic equations, Lecture Notes in Mathematics, 840, Springer-Verlag, (1980).

[16] Kato, T., Perturbation theory for linear operators, Classics in Mathematics, SpringerVerlag, (1995).

[17] PAZY, A., Semigroups of linear operators and applications to partial differential equations, Applied Mathematical Sciences, 44, Springer-Verlag, (1983).

[18] Rodríguez-Bernal, A., Localized spatial homogenizations and large diffusion, SIAM Journal of Mathematical Analysis, 29, 1361-1380,(1998). 


\section{Índice remissivo}

Aplicação dualidade, 9

Atrator global, 15

\section{Condição}

de crescimento, 30

de dissipatividade, 31

de estabilidade, 37

de sinal, 30

Cone finito, 21

Conjunto

$\alpha$-limite, 15

$\omega$-limite, 15

invariante, 15

resolvente, 8

Convergência

$X_{\varepsilon}^{\frac{1}{2}}-$ convergência, 42

$X_{\varepsilon}^{\frac{1}{2}}$-convergência fraca, 42

compacta, 45

Desigualdade

de Gronwall generalizada, 59

do Momento, 12

Espaço

de potência fracionária, 11

Espectro, 8

Estrutura gradiente, 18

Família coletivamente compacta, 45
Gerador infinitesimal, 8

Imagem numérica, 10

Medida de não-compacidade, 68

Operador

dissipativo, 9

adjunto, 10

auto-adjunto, 10

de potência fracionária, 11

setorial, 11

simétrico, 10

Orbita

completa, 14

negativa, 14

positiva, 14

Propriedade

de $C^{m}$ - regularidade uniforme, 22

do cone, 21

Semicontinuidade

inferior, 19

superior, 19

Semigrupo

analítico, 11

fortemente contínuo, 7

Solução, 13 
de equilíbrio, 64

Teorema

de Hille-Yosida, 9

de Lumer-Phillips, 9

de Rellich-Kondrakov, 21

de Schauder, 66

Variedade

estável, 18

instável, 18 